A

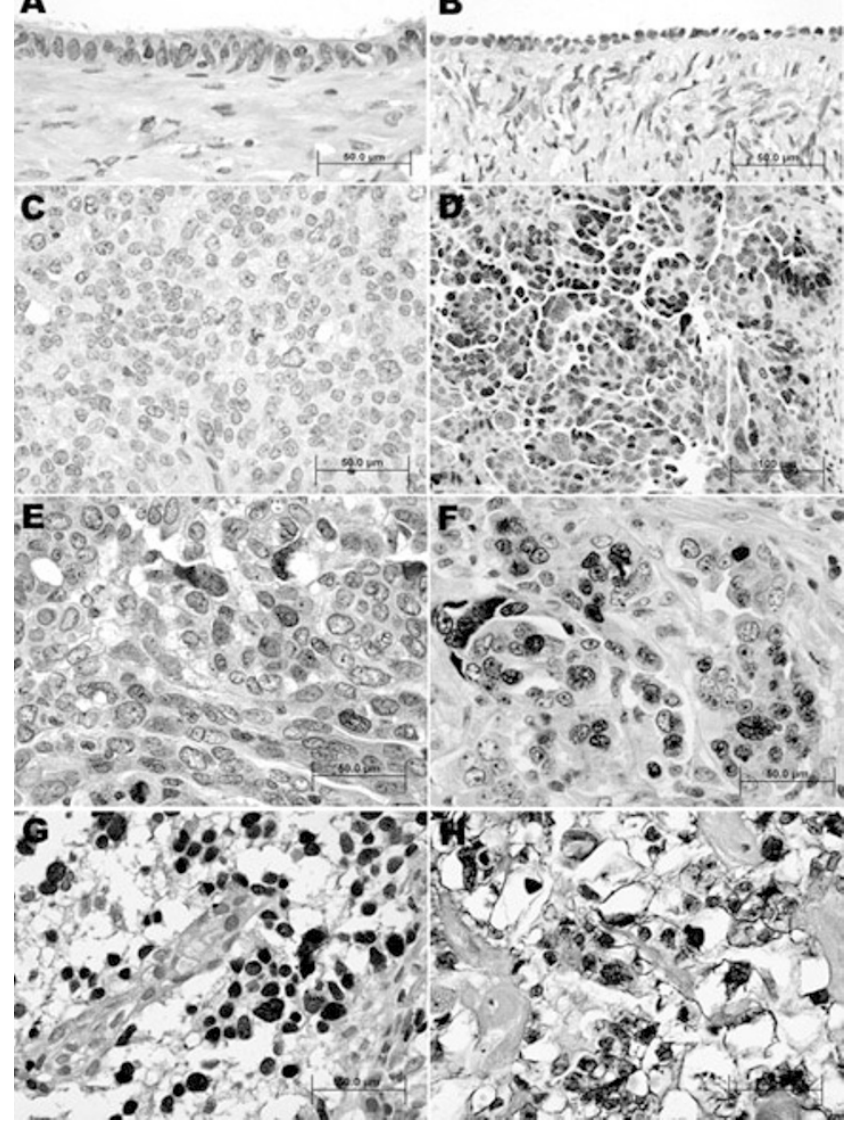

A Overall survival and SOX2 expression in BDisease-free survival and SOX2 expression in
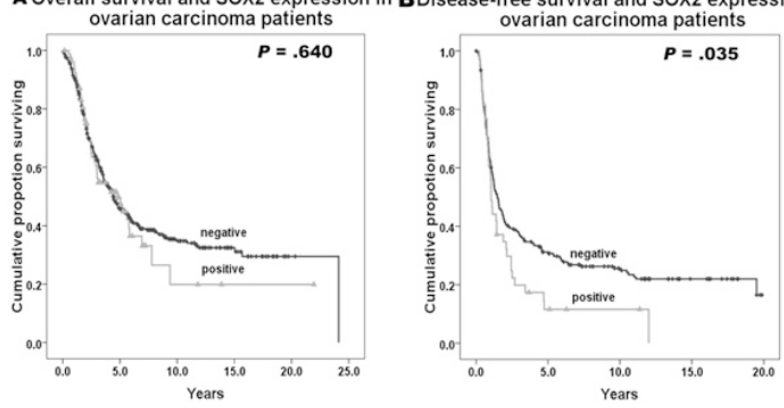

1277 Liquid-Based Cytology and High Risk HPV Screening Test Histories Preceding 2827 Histopathologic Cervical Intraepithelial Neoplasia 2/3 Diagnoses

C Zhao, M Amin, B Weng, X Chen, A Kanbour-Shakir, MR Austin. Magee-Womens Hospital of University of Pittsburgh Medical Center, Pittsburgh, PA; Conemaugh Valley Memorial Hospital, Johnstown, PA.

Background: Cervical screening in the US increasingly involves newer FDA-approved cytologic methods and adjunctive HPV testing. Since the primary goal of cervical screening is to prevent cervical cancer by identifying and treating precancerous cervical lesions, we decided to carry out a large scale audit of screening test histories for women with established histopathologic CIN2/3 diagnoses.

Design: A computer-based search of our CoPath database was conducted over a study period of 65 months between July 2005 and November 2010 to identify patients with histopathologic $\mathrm{CIN} / 3$ diagnoses. Preceding screening test histories for women screened with HC2 HPV testing and/or computer-imaged LBC ThinPrep Pap Tests were analyzed.

Results: 2827 patients with histopathologic CIN2/3 diagnoses were identified. The average patient age was 30.7 with a range of 16 to 91 years. Abnormal Pap tests $\leq 4$ months before histopathologic CIN2/3 diagnoses were identified in 2074 of 2827 patients (Table 1). HSIL was the most common abnormal result (41.6\%). Additional Pap test results, $\leq 3$ years before histopathologic $\mathrm{CIN} 2 / 3$ diagnoses, were identified in 1488 patients (Table 2). Prior HPV results $\leq 4$ months before CIN $2 / 3$ diagnoses were identified in 807 patients, with $97.4 \%$ hrHPV-positive. Additional HPV results, $\leq 3$ years before $\mathrm{CIN} 2 / 3$ diagnoses, were identified in 454 patients. $83 \%$ patients had at least one positive HPV results, but $24.2 \%$ had at least one negative HPV result.

Abnormal Pap Test Results Proximate to Histopathologic CIN2/3 Diagnoses
\begin{tabular}{|l|l|l||}
\hline Abnormal Pap & Case No & $\%$ \\
\hline HSIL & 862 & 41.6 \\
\hline LSIL & 464 & 22.4 \\
\hline ASC-US & 445 & 21.5 \\
\hline ASC-H & 288 & 13.9 \\
\hline AGC/AIS & 15 & 0.7 \\
\hline Total & 2074 & 100 \\
\hline
\end{tabular}

Prior 3 year-Pap Test History

\section{Pap Results}

Pap Results

At least one abnormal sme

At least one normal smear

Both normal and

Conclusions: 2074 of 2827 patients with histopathologic CIN2/3 had recent abnormal Pap results with HSIL in $41.6 \%$. $97.4 \%$ of 807 patients with CIN2/3 diagnoses and recent hrHPV results tested hrHPV-positive, most often after reflex testing and ASC-US Paps. Among patients with additional screening tests $\leq 3$ years before CIN $2 / 3$ diagnoses, a significant percentage of patients had negative Pap or negative HPV test results, consistent with rapid onset of at least some CIN2/3 lesions, inadequate sampling, or sensitivity issue of the tests. Our results support the value of co-testing in detection of small or inaccessible CIN2/3 lesions.

1278 Overexpression of Enhancer of Zeste Homolog 2(EZH2) and Focal Adhesion Kinase (FAK) - In High Grade Endometrium Carcinoma

J Zhou, S Bandyopadhyay, Z Chen, Y Hussein, B Alosh, T Jazaerly, K Hayek, R AliFehmi. Karmanos Cancer Center, Wayne State University, Detroit, MI.

Background: It has been reported that the deregulation of E-cadherin is associated with Src/FAK signaling axis and histone deacetylase (HDAC)/EZH2 activity. However, the association between EZH2 and FAK and the clinical significance of such an association in endometrial carcinoma has not been reported.

Design: 202 archived cases of endometrial carcinoma (1996-2000) were reviewed and divided into subtype (Type I and Type II). TMAs were developed as per established procedures. EZH2, FAK, pFAK immnuohistochemical stain were performed and expression was scored as negative (0), low (1) and high (2).

Results: A total 141 cases $(69.8 \%)$ Type I tumors and 61 cases $(30.2 \%)$ type II tumors were identified. The expression of EZH2 and FAK was detected in $68.8 \%$ and $46.1 \%$ of tumors, respectively, while pFAK was expressed in $64.7 \%$ of tumors. EZH2 overexpression was identified in $7.56 \%$ of type I tumors $v s .62 .96 \%$ of type II tumor $(\mathrm{p}<0.001)$. FAK and pFAK overexpression was only seen in $24.79 \%$ and $1.68 \%$ of Type I tumors as compared to $72 \%$ and $58.82 \%$ of type II tumors respectively $(\mathrm{p}<0.001)$. There was a positive correlation between the expression of EZH2, FAK and pFAK ( $p<0.0001$ for all paired association). The overexpression of EZH2, FAK, and pFAK were significantly associated with high histologic grade, angiolymphatic invasion, lymph node metastasis, myometrium invasion and cervical involvement (all $\mathrm{p}$ values $<0.01$ ). Kaplan-Meier curves showed that overexpression of EZH2 (Log-rank test, $\mathrm{p}=0.0024$ ), FAK and $\mathrm{pFAK}(\mathrm{p}=0.0001)$ were significantly associated with decreased overall survival. On multivariate analysis including the overexpression of EZH2, FAK and pFAK, tumor stage, tumor type, vascular invasion and tumor grade, only high tumor grade persisted as an independent prognostic factor for poor overall survival.

The Expression of EZH2, FAK and pFAK in Endometrial Carcinoma

\begin{tabular}{|c|c|c|c|c|c|c|c|}
\hline & & Type I (\%) & & & Type II (\%) & & p-value \\
\hline & Negative & Low & High & Negative & Low & High & \\
\hline EZH2 & $49(28.3)$ & $61(35.3)$ & $9(5.2)$ & $5(2.9)$ & $15(8.7)$ & $34(19.7)$ & $<0.001$ \\
\hline FAK & $88(52.7)$ & $29(17.4)$ & $0(0)$ & $2(1.2)$ & $12(7.2)$ & $36(21.6)$ & $<0.001$ \\
\hline pFAK & $54(31.8)$ & $63(37.1)$ & $2(1.2)$ & $6(3.5)$ & $15(8.8)$ & $30(17.6)$ & $<0.001$ \\
\hline
\end{tabular}

Conclusions: Our study thus suggests that overexpression of EZH2, FAK and pFAK correlate with several well established pathologic risk factors and might predict a more aggressive biologic behavior in endometrial carcinoma making them potential therapeutic targets for treatment of endometrial cancer.

\section{Head \& Neck}

1279 Primary Signet-Ring Cell (Mucin-Producing) Adenocarcinoma of Minor Salivary Glands: A Clinicopathologic, Immunohistochemical and Molecular Survey

JM Bastaki, BM Purgina, S Dacic, RR Seethala. University of Pittsburgh Medical Center, Pittsburgh, PA.

Background: Primary salivary signet-ring cell (mucin-producing) adenocarcinomas (SRCA) are extremely rare and poorly understood. We evaluate the clinicopathologic features of 4 cases and evaluate immunohistochemical and molecular features modeled after common profiles in mucinous/signet ring adenocarcinomas of other sites.

Design: Four cases were retrieved. Histochemical and IHC staining using standard technique was performed and included the following: mucicarime, PASD, AE1/3, CK7, CK20, CK5/6, CAM 5.2, CDX2, ER, AR, PSA, TTF-1, thyroglobulin, mammaglobin, HER2/neu, synaptophysin, chromogranin, actin, AMA, p63, calponin, PsAP, s100, GCDFP, Ki-67 and E-Cadherin. Additionally, fluorescence in situ hybridization for $A L K$ gene rearrangements using a break apart probe $2 \mathrm{p} 23$ was performed (Abbott Molecular, Des Plaines, IL). Cases with more than $20 \%$ of tumor cells showing a rearrangement were considered positive.

Results: The male:female ratio was $3: 1$. The mean age was 56 (range: 18-81). Sites involved were buccal mucosa (2), soft palate (1), and deep parotid (1). Perineural and angiolymphatic invasions were present in (3) and (2) cases respectively. The patient 
with a soft palate tumor had nodal metastasis on presentation. One patient was lost to follow-up, and the remainder, were alive and without disease at time of last follow up (mean 38 months). All cases showed a uniformly univacuolar (signet ring) appearance embedded in a myxoid stroma and were mucicarmine positive. The IHC profile is summarized in Table 1. Three cases showed at least focal p63 staining, and 2 cases were positive for calponin and GCDFP-15. Interestingly, membranous E-cadherin was retained and mammaglobin was positive in all cases. All tumors were negative for $A L K$ rearrangements.

\begin{tabular}{|c|c|c|c|c|}
\hline Case & 1 & 2 & 3 & 4 \\
\hline AE1/3 & + & + & + & + \\
\hline CK7 & + & + & + & + \\
\hline CAM 5.2 & + & + & + & + \\
\hline CK5/6 & + & + & - & - \\
\hline Mucicarmine & + & + & + & + \\
\hline PASD & + & + & + & + \\
\hline Calponin & + & + & - & - \\
\hline p63 & + & + & + & - \\
\hline s100 & - & + & - & - \\
\hline Mammaglobin & + & + & + & + \\
\hline GCDFP & - & + & - & + \\
\hline E-Cadherin & + & + & + & + \\
\hline Ki-67 & $5 \%$ & $5 \%$ & $60 \%$ & $60 \%$ \\
\hline
\end{tabular}

Negative stains are not shown

Conclusions: Alterations (i.e. loss of E-cadherin, $A L K$ rearrangements) noted in signet ring tumors at other sites are not present in salivary SRCA. The pathogenesis of this lesion is still elusive. The frequent coexpression of some myoepithelial markers and mammaglobin, mucicarmine and GCDFP-15 suggest a possible 'secretory myoepithelial' phenotype.

1280 Screening for HPV in Clinically Benign Tonsillectomy Specimens JB Bekker, MF Evans, KJ Threlkeld, V Rajendran, CS Adamson, K Cooper. University of Vermont, Burlington, VT

Background: The incidence of oropharyngeal squamous cell carcinoma is rising, as is the proportion of these tumors with detectable human papillomavirus (HPV). Vira etiology confers an improved prognosis as these tumors respond better to standard treatment when compared to HPV negative tumors. Many studies have evaluated the prevalence of HPV in tonsillar carcinomas, but few have investigated the prevalence of HPV in benign tonsils. We report our experience measuring the prevalence of HPV in non-neoplastic tonsillar tissue of patients 30 years and older.

Design: Two hundred tonsillectomy cases from adults aged 30 years and older were retrospectively reviewed from 2008 - 2010, each with a benign clinical indication fo surgery. A review of patient medical records showed no preoperative suspicion of malignancy in any case. Immunohistochemical staining for $\mathrm{p} 16^{\mathrm{INK} 4 \mathrm{a}}$ was performed on all cases as a marker for HPV infection. Confirmatory PCR and in-situ hybridization (ISH) for HPV was performed on all cases with positive p16 staining as well as cases with incidental dysplasia or carcinoma.

Results: Three specimens contained incidental squamous cell carcinoma and/or high grade dysplasia (1.5\%). Two of these tested positive for HPV, exhibiting strong, diffuse, nuclear and cytoplasmic p16 staining, confirmed by PCR and ISH. Of 197 cases with benign histology, all were negative for $\mathrm{p} 16$.

Conclusions: The prevalence of incidental malignancies and pre-malignancies in this study is much higher than previously reported, supporting the role of routine histological examination of tonsillectomy specimens in adults. Conversely, the presence of positive p16 staining in the absence of malignancy is much lower than previously reported; therefore testing should be reserved for cases with dysplastic or neoplastic epithelial changes.

1281 Clear Cell Odontogenic Carcinomas Show EWSR1 Rearrangements: A Novel Finding \& Biologic Link to Salivary Clear Cell Carcinomas

EA Bilodeau, I Weinreb, S Dacic, S Muller, B Barker, RR Seethala. University of Pittsburgh School of Dental Medicine, Pittsburgh, PA; University of Toronto, Toronto, ON, Canada; University of Pittsburgh, Pittsburgh, PA; Emory University, Atlanta, GA; University of Missouri-Kansas City, Kansas City, MO.

Background: Clear cell odontogenic carcinomas (CCOC) and salivary clear cell carcinomas (CCC) have considerable morphologic and immunophenotypic overlap making distinction difficult. LEF-1 is a transcription factor that is a marker of odontogenic differentiation that may serve to separate these entities. Recently, CCC have been noted to harbor an EWSR1-ATF1 translocation, which may potentially serve as a discriminatory marker. We performed a comparative analysis of $\mathrm{CCC}$ and $\mathrm{CCOC}$ for EWSR1 rearrangements and LEF-1 expression.

Design: Fluorescensce in-situ hybridization (FISH) for EWSR1 dual color break-apart 22q12 probe (Abbott Molecular, Des Plaines, IL) was performed on paraffin sections from $12 \mathrm{CCOC}, 14 \mathrm{CCC}$, and 1 sinonasal renal cell clear cell-like adenocarcinoma (SRCCA), 1 clear cell calcifying epithelial odontogenic tumor (CCCEOT), and 1 central squamous cell carcinoma with clear cell features (CCSCC). A case was considered FISH positive when $\geq 20 \%$ of the cells showed an EWSR 1 rearrangement. Immunohistochemical staining for LEF-1 (sc-8591, 1:200 dilution; Santa Cruz Biotechnology, Santa Cruz, CA, USA) was also performed and considered positive if over half the lesional cells showed at least moderate positivity.

Results: FISH testing failed in 4 CCOCs and 1 CCC. EWSR1 was rearranged in $62.5 \%(5 / 8)$ CCOC and $92.3 \%$ (12/13) CCC. All translocation positive CCC and CCOC showed clear cell tumor nests embedded in a characteristic juxtaposition of hyaline and fibrocellular stroma. One EWSR1 intact CCC was of the parotid but was morphologically identical to the EWSR1 translocated CCC. Two of the 3 EWSR1 negative $\mathrm{CCOC}$, however, had only sparse, compressed tumor islands embedded in a fibrocellular stroma. EWSR1 was intact in the SRCCA, CSCC, and CCCEOT. Immunohistochemistry for LEF-1 was only positive in 1/11 CCOC and 1/14 CCC. Conclusions: Most cases designated as CCOC demonstrate EWSR1 rearrangements suggesting that they are related entities to salivary CCC. Furthermore, LEF-1 is unable to serve as a discriminatory marker between these entities.

1282 Human Papillomavirus-Related Carcinomas of the Sinonasal Tract JA Bishop, TW Guo, DS Smith, H Wang, SI Pai, WH Westra. The Johns Hopkins Hospital, Baltimore, MD; Cleveland Clinic Lerner College of Medicine, Cleveland, OH. Background: High risk human papillomavirus (HPV) is an established cause of head and neck carcinomas arising in the oropharynx. The presence of HPV has also been reported in some carcinomas arising in sinonasal tract, but little is known about the overall incidence of HPV-related carcinomas of the sinonasal tract or the histologic features that characterize these tumors.

Design: We searched the surgical pathology archives of The Johns Hopkins Hospital for all carcinomas arising in the sinonasal tract from 1995 to 2011. We constructed tissue microarrays from 141 of the tissue blocks, and used whole slides for 37 additional cases. In situ hybridization for high-risk types of HPV was performed.

Results: Of 178 sinonasal carcinomas, thirty-five $(20 \%)$ were positive for high risk HPV. The HPV-related carcinomas occurred in 20 men and 15 women ranging in age from 33 to 87 years (mean 55). HPV-positive carcinomas consisted of 26 squamous cell carcinomas and variants (14 non-keratinizing, 4 papillary, 4 adenosquamous, 3 basaloid, and 1 keratinizing), 1 small cell carcinoma, 1 sinonasal undifferentiated carcinoma, and 7 carcinomas that were difficult to subtype by current classification schemes. These unclassifiable carcinomas were uniformly associated with high grade features (e.g. high mitotic rate, tumor necrosis), a basaloid component, a ductal componen reminiscent of salivary gland origin (e.g. tubular and cribriforming structures, stromal matrix deposition), and an immunohistochemical profile demonstrating the biphasic presence of ductal cells (c-kit and cytokeratin positive) and myoepithelial cells (S100, actin, calponin, and p63). Although these tumors were originally diagnosed as salivary gland neoplasms (e.g. solid variant of adenoid cystic carcinoma in 3 cases), the common presence of squamous dysplasia of the lining epithelium suggested surface origin. Conclusions: The presence of high risk HPV in $20 \%$ of sinonasal carcinomas suggest that it may play an important etiologic role for some carcinomas arising in the nasa passages. While non-keratinizing squamous cell carcinoma is the most common histologic type, there is a wide morphologic spectrum of HPV-related disease that includes a previously unrecognized high grade variant that is prone to confusion with salivary gland tumors, particularly the solid variant of adenoid cystic carcinoma.

1283 NUT Midline Carcinoma of the Sinonasal Tract

JA Bishop, WH Westra. The Johns Hopkins Hospital, Baltimore, MD

Background: NUT midline carcinoma (NMC) is a malignancy defined by translocations involving the $N U T$ gene on chromosome $15 \mathrm{q} 14$. NMC is important to recognize because it is highly lethal and because its chromosomal rearrangement offers the potential for targeted therapies. NMC involves midline structures including the head and neck. Six cases of the sinonasal tract have been reported, but its true incidence at this midline site is unknown because its morphologic features are not well known or recognized, and because sinonasal tumors are not routinely tested for the $N U T$ gene translocation. Translocations involving NUT result in expression of the NUT protein which is otherwise detected only in normal germ cells, and identification of NMCs has been facilitated by the recent availability of a highly sensitive and specific NUT immunostain.

Design: We searched The Johns Hopkins Hospital surgical pathology archives for all cases of sinonasal carcinoma from 1995 to 2011 and constructed tissue microarrays from the blocks. Immunohistochemistry with the NUT monoclonal antibody was performed. Results: Among the 141 primary sinonasal carcinomas tested, three ( $2 \%)$ NMCs were identified. Two of $12(17 \%)$ sinonasal undifferentiated carcinomas (SNUCs) and 1 of $80(1 \%)$ squamous cell carcinomas were revealed to be NMC. All occurred in men, aged 26,33, and 48 years (mean 36). The NMCs grew as nests and sheets of cells with abundant mitoses/apoptosis and necrosis. While two showed no histologic evidence of differentiation, one case had scattered "abrupt" nests of keratin. The nuclei of the NMCs were uniform, round to oval with open chromatin, and had one or more prominent eosinophilic nucleoli. Immunohistochemistry showed each carcinoma to be positive for cytokeratins and EMA. P63 was strongly positive in two, but was entirely negative in one. One of the cases was strongly CD34-positive, and the same case also showed focal positivity for neuroendocrine markers. Each patient died of his disease, with survivals of 8,11 , and 16 (mean 12) months despite combined surgery and chemoradiation. Conclusions: We found that NMCs comprise $2 \%$ of all sinonasal carcinomas including $17 \%$ of tumors previously classified as SNUC. Although we tested a variety of histologic types, the morphologic features of the positive cases conformed to those previously described for NMC. Although NMC is uncommon in the nasal passages, it should be considered in the differential diagnosis of a poorly differentiated malignancy of that site. Abrupt keratinization and nuclear uniformity in a high grade carcinoma are morphologic clues, and NUT immunohistochemistry is a useful confirmatory tool.

1284 Expression of Critical Genes Involved in EGFR-Signaling Pathway in HNSCC

A Bousamra, H Zhang, SA Schichman, C Fan. Univ Arkansas for Med Sciences, Little Rock, AR; Central Arkansas Veterans Healthcare System, Little Rock, AR.

Background: The Epidermal Growth Factor Receptor (EGFR) signaling pathway appears critically important in the pathogenesis of head and neck squamous cell carcinomas (HNSCC). Therefore, there has been immense interest in exploring targeted therapies aiming at EGFR, using either EGFR tyrosine kinase inhibitors (EGFR-TKI) or anti-EGFR monoclonal antibodies (EGFR-mab) for HNSCC. Because only a subset 
of HNSCC patients shows favorable responses to EGFR targeted therapy, correct selection of patient population who is destined to benefit from EGFR targeted therapy is important to improve the efficacy and efficiency of EGFR-targeted therapy in HNSCC. Design: Total RNA samples were extracted from 8 cultured HNSCC cell lines and 1 immortalized normal keratinocyte cell lines, followed by real-time RNA analyses for the following genes involved in the EGFR signaling pathway: EGFR, E-cadherin, PTEN and SCOS1.

Results: EGFR and PTEN genes are overexpressed in 7 out $8(87 \%)$ HNSCC lines with an average increase of 5.8 and 2.5 folds respectively when compared to normal keratinocytes. E-Cadherin gene is down regulated in 6 out of $8(75 \%)$ HNSCC lines with an average decrease by $61 \%$ when compared to normal keratinocytes. The average SOCS1 expression in HNSCC lines is slightly higher in 5 out of $8(62 \%)$ an average increase of 1.2 folds when compared to normal keratinocytes.

Conclusions: Overexpression of EGFR, PTEN and SOCS1 and downregulation of E-cadherin are common in HNSCC and a distinct EGFR signaling pathway gene expression pattern may be used to select for a subset of HNSCC responsive to EGFRtargeted therapies.

1285 Utility of Androgen Receptor, Her-2/Neu, Cytokeratin 5/6 and p63 Immunostaining in Distinguishing Salivary Duct Carcinoma from High Grade Mucoepidermoid Carcinoma

RT Butler, S Byrd, ME Spector, JB McHugh. University of Michigan, Ann Arbor, MI Background: Salivary duct carcinoma (SDC) can be misdiagnosed as high-grade mucoepidermoid carcinoma (MEC) (1). Distinguishing SDC from MEC is important as the former group of tumors is typically more clinically aggressive with poor 5-year survival rates. In addition, given high rates of androgen receptor (AR) positivity and Her-2/neu overexpression and amplification in SDC, targeted therapies are indicated in some patients. No systematic studies looking at immunohistochemical markers to distinguish these two entities have been performed.

Design: 48 cases of salivary duct carcinoma diagnosed at our institution were used to build a tissue microarray (TMA). This array, as well as a MEC TMA containing 81 carcinomas, including 9 high-grade tumors, were stained with antibodies to AR, Her-2/ neu, p63, CK 5/6 and k903 (34ßE12). The results of immunostaining (except for Her-2/ neu) were scored with a modified Allred score, utilizing intensity and percentage of tumor staining. Her-2/neu results were reported using an established scoring system. Sensitivity, specificity, positive predictive value (PPV) and negative predictive values (NPV) were calculated.

Results: Cytokeratin k903 was not helpful in distinguishing SDC from MEC as $84 \%$ and $98 \%$ were positive, respectively. AR and Her-2/neu positivity were specific for SDC (Table 1). In contrast, p63 and CK 5/6 were specific for MEC including the high grade MECs (Table 2). Three MECs that were negative or had only focal staining were low grade cystic and mucous cell rich and would not be confused with SDC.

SDC marker performance

\begin{tabular}{|c|c|c|c|c|}
\hline & Sens (\%) & Spec (\%) & PPV (\%) & NPV (\%) \\
\hline AR & 57 & 100 & 100 & 75 \\
\hline Her-2/neu & 22 & 100 & 100 & 69 \\
\hline
\end{tabular}

MEC marker performance

\begin{tabular}{|c|c|c|c|c|}
\hline & Sens (\%) & Spec (\%) & PPV (\%) & NPV (\%) \\
\hline p63 & 97 & 85 & 89 & 95 \\
\hline CK 5/6 & 84 & 65 & 75 & 76 \\
\hline
\end{tabular}

Conclusions: A panel of AR, Her-2/neu, p63 and CK 5/6 is helpful in distinguishing SDC from high grade MEC in cases where histologic overlap exists. This distinction is important as SDC is a highly aggressive malignancy with most patients dying of disease within 4 years of diagnosis. In addition, targeted therapies such as androgen deprivation and/or Herceptin may be indicated in SDC

1. Chevevert, Jacinthe, Leon E. Barnes, and Simion J. Chiosea. Mucoepidermoid Carcinoma: A Five-Decade Journey. Virchows Arch. 2011;458:133-140.

1286 Salivary Duct Carcinoma - Altered Pathways for Targeted Therapy $C$ Chattopadhyay, ME Kupferman, MS Kies, MD Williams. UT MD Anderson Cancer Center, Houston, TX.

Background: Salivary duct carcinoma (SDC) is a high grade adenocarcinoma with median survival of less than three years. Identifying targetable pathways are needed for improved outcomes in this aggressive disease. Novel inhibitors of PI3K currently in clinical trials attempt to suppress misregulation which may be caused by loss of Phosphatase and tensin homolog (PTEN) expression, a tumor suppressor gene. Whereas direct inhibitors of c-MET, a proto-oncogene associated with motility, and proliferation, are also being studied which actives multiple pathways including that of PI3K. As little is known regarding these potential targetable pathways in salivary duct carcinoma, we sought to define their incidence and correlate with clinical parameters.

Design: A tissue microarray composed of formalin-fixed paraffin embedded tumor tissue from 62 salivary duct carcinomas formed the study cohort. Immunohistochemical analysis was performed for PTEN, c-MET and p-MET expression using standard techniques (PTEN 6H2.1, 1:100, Dako, Carpenteria, CA; c-MET, sc-10, 1:200, Santa Cruz). Each tumor was evaluated for expression in duplicate cores for: PTEN expression- negative or positive (cytoplasm and/or nuclear) and c-MET/ $\mathrm{p}-\mathrm{MET}$ as 0 (no expression), $1+$ (weak), $2+$ (moderate) and $3+$ (strong) staining.

Results: Within the 62 SDCs, PTEN loss of expression was seen in $22(34 \%)$, a similar incidence as seen in breast adenocarcinoma. C-MET showed high $(2$ or $3+)$ expression in $31(50 \%$ ) of the SDCs and high p-MET expression was present in $27(43 \%)$ tumors. High-p-MET occurred in SDCs with all ranges of c-MET expression (0-3+). No correlation was noted between clinical outcome and any of the markers analyzed.
Conclusions: Alterations in PTEN and MET expression occur in over a third of salivary duct carcinomas and may allow for targeted therapies through inhibition of the PI3K pathway and MET directly. Studies are on-going to delineate the molecular basis for these alterations.

1287 Oncofetal Protein IMP3, a New Diagnostic Biomarker for Laryngeal Carcinoma

$K$ Chen, K Cornejo, W Ye, Q Wu, J Liang, Z Jiang. State Key Laboratory of Oncology in South China, Guangzhou, Guangdong, China; Cancer Center, Sun Yat-sen University, Guangzhou, Guangdong, China; University of Massachusetts Medical School, Worcester, MA.

Background: An accurate diagnosis of laryngeal squamous cell carcinoma (LSCC) is essential for patient management. The diagnosis of LSCC, especially in superficial biopsies can present a diagnostic challenge for pathologists. The ability to diagnose LSCC would be greatly improved by the detection of a tumor-associated antigen. IMP3, an oncofetal protein plays an important role in embryogenesis and carcinogenesis of certain malignant neoplasms. The aim of this study was to investigate the expression of IMP3 in LSCC and to determine whether it can serve as a diagnostic biomarker for LSCC.

Design: A total of 227 cases (laryngectomy, $n=107$; biopsy, $n=120$ ) of LSCC were obtained from the surgical pathology files of a tertiary Medical Center between 2000 and 2005. The diagnoses of all the cases were confirmed by at least two pathologists. Results: IMP3 showed strong cytoplasmic staining in 210 of 227 (93\%) LSCCs. IMP3 positivity was detected in $>50 \%$ of tumor cells in $156(74 \%)$ cases, $26-50 \%$ of tumor cells in $18(9 \%)$ cases and $<25 \%$ of tumor cells in $36(17 \%)$ cases. IMP3 expression was detected in 78 of $88(89 \%)$ well-differentiated, 99 of $101(98 \%)$ moderatelydifferentiated and 35 of $38(92 \%)$ of poorly-differentiated LSCC. In contrast, the expression of IMP3 was undetectable in all adjacent benign squamous epithelium, including pseudoepitheliomatous hyperplasia.

Conclusions: Our findings indicate that IMP3 is a highly sensitive and specific positive biomarker for LSCC. IMP3 is highly expressed in LSCC but not in adjacent benign squamous epithelium, which suggests IMP3 may play an important role in the pathogenesis of LSCC as it has been shown to play a role in cell proliferation, invasion and migration.

1288 Epigenetic Modulation of EGFR-Signaling Genes in HNSCC

LN Clark, H Zhang, SA Schichman, C Fan. Univ Arkansas for Med Sciences, Little Rock, AR; Central Arkansas Veterans Healthcare System, Little Rock, AR.

Background: Epidermal Growth Factor Receptor (EGFR) signaling pathway appears critically important in the pathogenesis of head and neck squamous cell carcinomas (HNSCC). For the above reason, there has been immense interest in exploring targeted therapies aiming at EGFR, using either EGFR tyrosine kinase inhibitors (EGFR-TKI) or anti-EGFR monoclonal antibodies (EGFR-mab) for HNSCC. Characterization of epigenetic regulation of EGFR signaling genes (EGFR, E-Cadherin, PTEN and SCOS1) may lead to more effective treatment of HNSCC using EGFR-targeted therapies. Design: Promoter sequences of 4 EGFR signaling genes (EGFR, E-cadherin, PTEN and SCOS1) were analyzed for the presence or absence of CPG island using CpG Plot software. Total RNA samples were extracted, followed by real-time RNA analyses for these 4 genes from 8 cultured HNSCC cell lines, treated with or without a DNA demethylating agent, 5-aza-cytidine.

Results: All 4 EGFR signaling genes (EGFR, E-cadherin, PTEN and SCOS1) contain a CpG island in their respective promoter sequence, ranging from 233 to 602 basepairs. Following treatment with a DNA methylation inhibitor, 5-azacytidine, increased expression of EGFR, E-cadherin, PTEN and SCOS1 genes was seen in 4/8 (50\%), $4 / 8(50 \%), 1 / 8(12 \%)$ and $5 / 8(62 \%)$ HNSCC lines respectively as compared to their original cells without 5 -aza cytidine treatment

Conclusions: The presence of a $\mathrm{CpG}$ island in the promoter region and increased gene expression in response to DNA demethylation support that the expression of 3 EGFR signaling genes (EGFR, E-cadherin, SCOS1) may be suppressed by promoter hypermethylation and these genes can be unregulated by DNA demethylating agent in HNSCC. Therefore, DNA demethylating agent may be used to improve the response rate for HNSCC treated with EGFR-targeted agents (EGFR-TKI or (EGFR-mab).

1289 Differential microRNA Expression Signatures in Salivary Duct Carcinomas Versus Her2/Neu 3+ Positive Hormone Receptor Negative Invasive Ductal Breast Carcinomas and High Grade Breast Ductal Carcinoma In Situ

$S$ Costinean, A Bottoni, L Cascione, T Teknos, E Ozer, M Old, A Agrawal, CM Croce, $H$ Iwenofu. The Ohio State University, Columbus.

Background: Salivary duct carcinomas (SDC) are highly lethal tumors that are histologically and immunohistochemically indistinguishable from invasive high grade Her2/Neu positive hormone receptor negative ductal breast carcinomas (IDBC). MicroRNAs (miRs)s are a newly discovered class of noncoding RNAs with roles in posttranscriptional regulation. Emerging data in hematologic and solid tumors indicate that there are miR expression "genetic signatures" that could be used for diagnosis, prognosis or therapy.

Design: We investigated whether the common histopathologic and immunophenotypic features of SDC and Her2/Neu 3+ hormone receptor negative IDBC correspond to a similar miR "signature". 5 cases of SDC, 6 cases of IDBC Her/neu2 $3+$ and 4 cases of high grade ductal breast carcinoma in situ (DCIS) were selected from paraffin embedded blocks from the OSU tissue archive. Representative areas of tumor were cored. RNA was isolated with Recover easy from Ambion and analyzed using Nanostring technology. The results were processed using SAM and cluster analysis module. To validate Nanostring 
results TaqMan Assays were used. The comparative $\mathrm{C}_{\mathrm{T}}$ method for relative quantitation of gene expression was used to determine miR expression levels.

Results: The expression patterns of Her2/Neu 3+ IDBC and SDC were strikingly similar and much less so with high grade DCIS. We found that compared to SDC, Her/neu3+ IDBC had higher levels of miR142-3p and miR10a. When compared to SDC, DCIS had higher levels of miR10a also, together with miR99a, miR335 and miR331-3p and lower levels of miR19a and miR15a. All results were validated by Real Time PCR. Conclusions: A striking conclusion was that SDCs are closer to Her2/neu 3+ IDBC than the high grade DCIS. Only 2 miRs were differentially expressed compared to IDBC and 4 miRs differentially expressed compared to the high grade DCIS. One of the miRs differentially expressed - miR10a - was consistently higher in both high grade DCIS and Her2/Neu 3+ IDBC compared to the SDCs suggesting that this miR is breast specific and its expression is increased early in tumorigenesis.

1290 Expression and Prognostic Significance of Directed Therapy Targets and Mutational Analysis of the Egfr Pathway in Malignant Salivary Gland Tumors

JFE Cros, H Blons, E Sbidian, E Tartour, S Hans, D Brasnu, P Bruneval, C Badoual. Georges Pompidou European Hospital, Paris, France; Paris Est Creteil University, Creteil, France; INSERM - Paris Descartes University, Paris, France.

Background: Malignant salivary gland tumors are rare and pleomorphic entities (24 sub types, WHO 2005). Curative treatment relies on surgery sometimes completed by radiotherapy. Management of non-surgical, locally advanced or metastatic tumors is difficult as conventional chemotherapies are ineffective. Innovative treatments such as directed therapies may provide important benefits for these patients. The aim of this work was to assess the expression and prognostic value of directed therapy targets on salivary gland tumors and search for mutations within the key players of the EGFR pathway. Design: Immunochemistry on formalin fixed paraffin embedded (FFPE) samples from 124 patients for c-KIT, EGFR, c-ERBB2, MUC1, phospho-mTOR, androgen/estrogens/ progesterone receptors and Ki67 was performed and related to progression free interval and survival. DNA was extracted from FFPE sample and mutational status of EGFR/ KRAS/ BRAF/HRAS were assessed.

Results: EGFR was the most expressed marker, found across almost all histotypes. Expression of the other markers was heterogeneous, depending on the tumor sub type. C-ERBB2, c-KIT and Ki67 were associated with the tumor grade. Tumor grade and a high proliferation index $(\mathrm{Ki} 67>20 \%)$ were associated with the progression free interval and survival. None of the other marker was. No mutation was found in EGFR/KRAS/ BRAF. 7\% (7/104) of the tumors harbored a mutation at the codon 61 of HRAS. In epithelial and epithelial-myoépithélial carcinoma, the mutation rate reached $33 \%$. Conclusions: Several tumor subtypes expressed frequently some targets of directed therapies suggesting potential therapy leads. For instance, high levels of C-ERBB2 and androgen receptors in salivary duct carcinomas or C-KIT over expression in myoepithetial carcinomas were found. The EGFR pathway seems intact suggesting a low efficacy of small kinase inhibitors such as erlotinib or gefitinib. The mutation of H-RAS, known to be important but not sufficient to trigger urothelial carcinoma, calls for a broader screen to identify the pathways involved in malignant salivary gland tumors carcinogenesis.

1291 HPV16/18 + Oropharyngeal Carcinomas Are Associated with Enhanced Local Adaptive Immunity

$Q$ Dai, T Isayeva, $J$ Xu, M Brandwein-Gensler. $\mathrm{UAB}$, Birmingham, $\mathrm{AL}$

Background: HPV promotes many oropharyngeal carcinomas (OPC) and some oral cavity carcinomas (OCC). A meta-analysis found $36 \%$ of OPC are HPV+, mostly HPV16. OCC are also associated with HPV, but a lesser degree. Outcomes for OPC $\mathrm{HPV}+$ patients are significantly better than HPV negative patients, the mechanisms for this protective effect are being elucidated. In-vitro evidence show that HPV induces immune tolerance. But clinical data support the idea of HPV-specific immunity. We hypothesize that $\mathrm{HPV}+$ carcinomas are associated with enhanced local adaptive immunity.

Design: We studies consecutive patients resected for OCC $(n=44)$ and OPC $(n=$ 34). Tumor RNA was extracted from blocks, reverse transcription was performed. Nested quantitative RT-PCR was performed with primers to HPV16 (E6 (including E6*)/E7) and HPV18 (E6/E7). Positive and negative controls (SiHa, HeLa, HPV16+ OPC, and pancreatic carcinoma, water, respectively) were included in all reactions. Tumors were classified as HPV+ only if both concordant E6 and E7 transcripts were present. One tissue section was selected from each case with the strongest cancerassociated lymphocytic response, and double-stained, by immunohistochemistry, for CD4/CD8 (cocktail antibody, ThermoScientific). Whole slide scanning was performed (BioImagene); 10 40X fields were selected from the regions of densest tumor-infiltratinglymphocytes (TILS), and densest immune response at the tumor-interface. CD8/CD4 cells were quantified as percent cells per 40X field. CD8/CD4 cells were correlated with HPV16/18 status by T-test, comparing mean percentages of CD8/CD4 cells per distribution (TIL, interface), per site (OCC, OPC), per HPV status.

Results: Transcriptionally active HPV16/18 was present in 20/34 (59\%) of OPC, and 16/44 (36\%) OCC. Cytotoxic CD8 cells were significantly increased in HPV+ OPC, both TILS, and at the interface. Interface CD4 cells were also significantly increased (Table 1). No increase in adaptive immunity was seen in HPV+ OCC (data not shown). Conclusions: We show that HPV16/18+ OPC are significantly associated with enhanced local adaptive cytotoxic immunity, compared the HPV- OPC. Thus enhanced immunity may constitute another protective mechanismfor HPV-mediated OPC.

\begin{tabular}{|c|c|c|c|c|c|}
\hline $\begin{array}{l}\text { Cell of } \\
\text { Interest }\end{array}$ & Distribution & $\begin{array}{l}\text { HPV 16/18 } \\
\text { Status }\end{array}$ & $\begin{array}{l}\text { Mean } \\
\text { Cell } \\
\%\end{array}$ & Sig (2-tailed) & $95 \%$ CI $\Delta$ Mean Cell $\%$, lower, upper \\
\hline \multirow[t]{4}{*}{ CD8 } & Interface & Pos & 21.1 & 0.024 & 19.5 \\
\hline & Interface & Neg & 10.6 & & \\
\hline & Intratumor & Pos & 10.4 & 0.002 & 11.9 \\
\hline & Intratumor & Neg & 3.1 & & \\
\hline \multirow[t]{4}{*}{ CD4 } & Interface & Pos & 21.6 & 0.012 & 21.4 \\
\hline & Interface & Neg & 9.5 & & \\
\hline & Intratumor & Pos & 3.8 & 0.439 & -1.8 \\
\hline & Intratumor & $\mathrm{Neg}$ & $\begin{array}{l}2.6 \\
2.6\end{array}$ & & \\
\hline
\end{tabular}

1292 Transcriptionally Active High-Risk HPV: Uncommon in Oral Squamous Carcinomas

Q Dai, JXu, TIsayeva, D Dan, G Jour, M Vered, B Wenig, M Brandwein-Gensler. UAB, Birmingham, AL; Tel-Aviv University, Tel-Aviv, Israel; Continuum Health Partners Beth Israel Medical Center, New York, NY.

Background: Transcriptionally active high-risk Human Papillomavirus (HR-HPV) is important in promoting many oropharyngeal carcinomas (OPC). HR-HPV-mediated OPC are associated with significantly improved outcomes, forming the basis for new treatment paradigms. Few large-scale studies have looked at HR-HPV genome detection rate in oral squamous carcinoma (OSC) by PCR. No prior study has examined the transcriptional activity of HPV in OSC; this speaks to the issue of biologically relevant, versus bystander infection. Here, we look for the presence of transcriptionally active HR-HPV in 87 patients with OSC.

Design: RNA was extracted from archival OSC resections from three academic centers. Reverse transcription, and nested real-time PCR (RT-PCR) were performed with separate primers to HPV16 E6 (including E6*)/E7 and HPV18 E6/E7. Tumors were categorized as positive if both $\mathrm{E} 6$ and $\mathrm{E} 7$ transcripts were detected. Positive controls ( $\mathrm{SiHa}, \mathrm{HeLa}$, archival HPV16+ oropharyngeal SCC) and negative controls (HeLa, pancreatic carcinoma, and no tissue) were included in all reactions, which were run in duplicate. Tumors were classified as HPV+ only if both concordant E6 and E7 transcripts were present. Data on demographics (age, gender, stage, treatment, alcohol (Yes/No), smoking exposure (Yes/No)) and outcome (disease-progression, disease-specific survival) were collected. Fisher's exact test was used to analyze HPV status and demographics; Kaplan Meier curves were to analyze HPV status and outcome.

Results: This cohort consisted of 49 males (56\%) and 38 females (44\%) with a mean age of $61.3 \pm 12.8$ years (range, 27-89). Alcohol and smoking exposures were present in $19 / 58(33 \%)$ and $39 / 68(57 \%)$ of patients, respectively. HPV16 transcripts were detected in 16/87 (18.4\%) of OSC. There were no significant correlations with HPV16 status and patient age, gender, alcohol, or smoking exposure. There were no differences in time to disease-progression or disease-specific survival based on HPV16 status. HPV18 transcripts were detected in 12/47 (25.5\%) of OSC. HPV18 studies are ongoing.

Conclusions: Transcriptionally active HPV16/18 infections are uncommon in OSC. There is no evidence to suggest that transcriptionally active HPV16 infection confers a large survival advantage for oral cancer patients, as has been demonstrated in the oropharynx. However, this study is not powered to observe smaller differences in outcome based on HPV status, neccesitating further study.

\section{Nodular and Intravascular Fasciitis of the Lip}

WA Daraji, ELB Childers, JC Fanburg-Smith. Armed Forces Institute of Pathology, Washington, DC; Howard University, Washington, DC; Sibley Memorial Hospital of Johns Hopkins Medicine, Washington, DC.

Background: Nodular or intravascular fasciitis of the lip are exceedingly rare, with few singly reported cases.

Design: All oral nodular fasciitis were culled. The patient slides, anatomic locations, and histories were reviewed.

Results: There were 7 cases in the lip, 2 nodular and 5 intravascular fasciitis, 3 of the upper and 4 lower lip, including 6 females and one male. Patient ages ranged from 43-72 years, median 8 years. All patients were smokers and 5 habitually bit their lips. The lesions, $1-2.1 .0 \mathrm{~cm}$ (median $1.1 \mathrm{~cm}$ ) arose rapidly over 3 weeks to 3 months. These nodular masses were circumscribed $(n=4)$ or poorly delineated, three involving lip skeletal muscle. The lesions were composed of loose storiform tissue culture like myofibroblasts with myxoid degeneration and extravasated red blood cells. The five intravascular fasciitis additionally had focal myoid/myopericytic features, associated with vessel wall/lumen. None had osteoclast type giant cells, myofibroma or angiomyoma features. All lesions were recurrent between 1 and 11 months (median 4 months) after original removal, but none had any other disease at 12-22 years (median 16 years) follow-up.

Conclusions: Intravascular fasciitis is a subset of nodular fasciitis with a proclivity for lip, in adult females who smoke and lip-bite. These differ from nodular or intravascular fasciitis in other soft tissue sites by their superficial skeletal muscle involvement and the focal myopericytic features associated with intravascular type. Lip intravascular fasciitis may be recurrent, possibly due to continued habitual lip biting and/or smoking, but is ultimately a benign reactive process, probably secondary to mechanical irritation of vessel wall in the lip.

1294 The Prevalence of High-Risk HPV in Aerodigestive and Lung Cancers in HIV+ Patients

TM Deal, T Isayeva, J Willig, M Brandwein-Gensler. University of Alabama, Birmingham, AL.

Background: The incidences of lung cancer and head and neck squamous cell carcinoma (HNSCC) are significantly higher in patients infected with the human immunodeficiency virus (HIV). Previous studies suggest that high-risk Human Papillomavirus (HR-HPV) 
may be important in the pathogenesis of lung cancer in immune-competent individuals. The prevalence of HR-HPV in lung cancers of HIV+ patients has not yet been studied, and only two small cohorts of HIV+ patients with HNSCC have been studied for HR-HPV. It is important and novel to study a large cohort of HIV+ patients with these cancers as there are therapeutic implications for HR-HPV-mediated cancers.

Design: The UAB databases for the outpatient AIDS clinic and pathology department were queried. The diagnoses of HIV were confirmed by CD4-counts and/or viral loads. The histological diagnoses were reviewed and confirmed. Nucleic acids were extracted from archived specimens. Only specimens with positive internal nucleic acid controls (GAPDH) were studied further for HPV. Nested polymerase chain reactions (PCR) were performed with GP5/6 consensus primers. Reverse transcription and nested real-time PCR (RT-PCR) were performed with HPV16/18 E6/E7 primers. Tumors were classified as positive only if both concordant E6/E7 reactions were positive. Positive controls (HeLa, SiHa, HPV16+ HNSCC) and negative controls (cirrhotic liver, pancreatic, colonic, renal carcinomas and water) were included in all reactions, which were run in duplicate.

Results: We identified $14 \mathrm{HIV}+$ patients (11 males, three females) ages 26-67 (median 49.5 years). Seven patients had lung cancer (adenosquamous), and 7 had HNSCC (5 oral cavity, 2 larynx). Thirteen tumors were positive using the GP5/6 primers, with appropriately reactive controls; the agarose gel bands demonstrated slightly different sizes, as is expected, indicating different HPV-types. Two tumors were HPV16+ (1 lung, 1 larynx), 4 tumors were HPV18+, ( 3 lung, 1 oral cavity), 6 tumors were HPV16/18+ ( 3 lung, 2 oral cavity, 1 larynx). One oral cavity cancer was positive by GP5/6 primers, but negative for HPV 16/18, indicating a different HPV-type. One tumor was HPV18+ (oral cavity); results are pending for HPV16 and GP5/6.

Conclusions: This preliminary study indicates that transcriptionally active HPV16/18 is common in HNSCC and lung cancers for this population, which may be an important disease-modifying factor. Future goals include the study of a larger cohort, with matched HIV-negative patients.

\section{EBV Prevalence in Nasopharyngeal Carcinoma}

$S$ Dogan, SI Chiosea. Memorial Sloan-Kettering Cancer Center, New York, NY; University of Pittsburgh Medical Center, Presbyterian Hospital, Pittsburgh, PA. Background: Nasopharyngeal carcinoma (NPC) is an epithelial malignancy of multifactorial etiology, including Epstein-Barr virus (EBV) and cigarette smoking. To characterize the morphologic evolution of NPC over the last 60 years, we compared basic demographic and morphologic features and prevalence of EBV in two cohorts of NPC: those diagnosed from 1957 to 1977 and cases from 2002 to 2010.

Design: NPC cases diagnosed in non-Asian patient population from 1957 to 1977 (Group 1, N=29) and from 2002 to 2010 (Group 2, N=28) were reviewed and categorized according to WHO 2005 classification as keratinizing (K-NPC) or non-keratinizing (NKNPC) and tested for presence of Epstein-Barr virus early RNA by in situ hybridization (EBER-ISH). All cases from Group 1 were tested for total RNA integrity.

Results: Most NPC patients in Group 1 and Group 2 were men $(25 / 29,86.2 \%$ and $20 / 28,71.4 \%$ ). The average age at presentation was similar in both cohorts (53 yrs, Group 1 and 54 yrs, Group 2). K-NPC was more frequent in Group 1 than in Group 2 $(10$ of $29,34.5 \%$ vs. 3 of $28,10.7 \%$; odds ratio $=2.9,95 \%$ confidence interval 0.7 $11.6 ; \mathrm{p}=0.03$ ). Total RNA quality was acceptable in 24 of 25 cases (Group 1). Change in the predominant morphology (from $\mathrm{K}$ to $\mathrm{NK}$ ) was associated with slight increase in EBER ISH positivity in Group 2 ( 15 of $24,51.7 \%$, Group 1 vs. 18 of $25,62.3 \%$, Group 2, $\mathrm{p}=0.24$ ).

Conclusions: The predominant morphologic type of NPC changed from keratinizing to non-keratinizing. Patients' gender, age at diagnosis, prevalence of EBV did not change significantly. Decrease in K-NPC over the last several decades may suggest diminished impact of non-EBV-related NPC etiologies such as smoking.

1296 Prevalence of Human Papillomavirus in Squamous Cell Carcinoma of the Oral Cavity with Correlation of p16 Immunohistochemistry and Human Papillomavirus Polymerase Chain Reaction

LD Duncan, $M$ Winkler, S Lee. University of Tennessee Graduate School of Medicine, Knoxville, TN

Background: Human Papillomavirus (HPV) has been identified in cases of head and neck squamous cell carcinoma, suggesting an etiologic role. HPV type 16 is the most common serotype, and oropharyngeal tumors are more frequently HPV positive. The oncogenic properties of HPV 16 are the result of HPV host cell genome integration, resulting in increased production of E6 and E7 oncoproteins, inactivation of retinoblastoma protein and p16 over expression. In our study, we examine the clinical utility of p16 immunohistochemistry (IHC) in detecting HPV in oral cavity squamous cell carcinoma (OCSCC). Through comparison with results of HPV polymerase chain reaction (PCR), the use of $\mathrm{p} 16 \mathrm{IHC}$ as a surrogate marker for HPV is investigated.

Design: 83 cases of OCSCC from the tongue, floor of mouth, buccal mucosa, hard palate, and gingiva were included in the study. Oropharyngeal tumors were excluded. Non-decalcified paraffin embedded tumor from each case was subjected to $\mathrm{p} 16 \mathrm{IHC}$ and HPV PCR. Results of IHC and HPV PCR were compared. The intensity of p16 staining was graded 0 to $3+$, and the location of staining in tumor sections and individual tumor cells was recorded. The histologic appearance of HPV positive tumors was documented to elucidate potential patterns associated with HPV positivity.

Results: 8 of 83 cases $(9.6 \%)$ were PCR positive, all HPV serotype 16 . All of these cases had $3+$ nuclear and cytoplasmic p16 staining. 42 tumors (50.6\%) were completely p16 negative. 25 cases $(30.1 \%)$ had $1+$ p16 staining, including 8 cases with faint staining in benign epithelium adjacent to tumor. 8 cases $(9.6 \%)$ had $2+$ p16 staining. All of the HPV PCR positive cases had histologic evidence of keratinization.
Conclusions: In our study, strong cytoplasmic and nuclear staining for p16 (3+) correlates with HPV PCR positivity. This pattern of p16 staining can be used as a surrogate marker for high risk HPV in lieu of HPV PCR. The presence of 1 to $2+\mathrm{p} 16$ staining in tumor and adjacent benign and dysplastic tissue is due to HPV independent mechanisms of p16 over expression since all of these cases were HPV PCR negative. HPV PCR positive study cases displayed varying amounts of keratinization typical of oral cavity location and not the basaloid growth pattern described in HPV positive tumors in prior studies, most of which were in an oropharyngeal location. This observation confirms that a basaloid tumor growth pattern does not exclusively correlate with HPV positivity.

1297 Mucinous Myoepithelioma, a Previously Unrecognized Variant CJ Esteva, LJ Slater, DR Gnepp. Warren Albert Medical School of Brown University, Rhode Island Hospital, Providence, RI; Scripps Oral Pathology Service, San Diego, CA. Background: Myoepithelial neoplasms are salivary gland tumors composed almost exclusively of cells with myoepithelial differentiation that may exhibit spindle, plasmacytoid, epithelioid or clear cytoplasmic features. They are uncommon accounting for $1.5 \%$ of benign $\&<2 \%$ of malignant salivary gland tumors. We have encountered a subset of confirmed benign \& malignant myoepithelial tumors containing intracellular mucin. This represents a unique, previously undescribed subtype that does not fit in the current classification system. This paper presents 2 unique myoepitheliomas with prominent intracellular mucin

Design: 29 myoepithelial neoplasms from our surgical pathology, teaching \& consult archives from 1997-2011 were reviewed for the presence of intracellular mucin.

Results: 18 adenomas \& 11 carcinomas were reviewed from patients 28-87 years old; 17 women \& 12 men. All myoepithelial tumor cell types \& growth patterns were included. Cases were reviewed by 2 authors for the presence of intracellular mucin; first by H\&E, then by Mucicarmine ( 26 cases) and Periodic Acid Schiff (PAS) with \& without diastase (19 cases). Most cases examined showed mild to prominent extracellular/stromal mucin, without evidence of intracellular mucin. Two tumors had abundant intracellular mucin with H\&E staining, confirmed by mucicarmine \& PAS diastase staining in 1 case and by PAS diastase only in the other. The benign case arose in the parotid gland of a 69yo male. It was encapsulated, composed of sheets of closely packed nests $\&$ trabeculae of plasmacytoid to polygonal cells compartmentalized by slender fibrovascular septa. Tumor cells had abundant eosinophilic to foamy grayish-blue cytoplasm, frequently containing intracellular mucin, mild nuclear pleomorphism \& fine peppery chromatin with inconspicuous nucleoli. It was positive for cytokeratin, SMA, S100 \& GFAP. The $2^{\text {nd }}$ tumor arose in the hard palate of a $75 y$ o female. It was multinodular, wellcircumscribed, focally invasive $\&$ was composed of nests $\&$ sheets of cells with moderate nuclear pleomorphism, prominent nucleoli \& abundant lightly eosinophilic cytoplasm with a focally prominent plasmacytoid-type configuration. Throughout much of the tumor were areas with prominent intra-cytoplasmic mucinous-type secretions \& focal areas with extracellular mucin material. It was positive for Calponin, Cytokeratin, S100, CK7, CEA \& GFAP

Conclusions: We propose a new variant of myoepithelioma, mucinous type, in which there is focal or abundant gray to bluish intracellular mucin

1298 Proximal-Type Epithelioid Sarcoma of the Head and Neck (HN): A Study with Immunohistochemical and Molecular Tests

R Frank, N Sadri, T Bhatti, JA Biegel, VLiVolsi, PJ Zhang. Hospital of the University of Pennsylvania, Philadelphia; Children's Hospital of Philadelphia, Philadelphia. Background: Proximal epithelioid sarcoma (PES) is an aggressive variant of epithelioid sarcoma most often occurring in soft tissue of proximal limbs and characterized by polygonal cells, marked nuclear atypia, and varied rhabdoid features. Morphologic and immunohistochemical (IHC) similarities between PES and malignant extra-renal rhabdoid tumors (MRT) pose a diagnostic challenge and may suggest a relationship between the two. Both tumors are extremely rare within the head and neck and both are characterized by loss of INII/SMARCBI expression. The molecular events involving $S M A R C B 1$ seem to differ however, with point mutations more common in MRT and deletions in PES.

Design: Three PES of $\mathrm{HN}$ were evaluated. The patients were ages 9, 43, and 71 years old, and tumor locations were posterior para-median neck, sinonasal, and retro-orbital respectively. Histologic and IHC sections were evaluated. Mutation analysis by direct sequencing of the promoter and 9 exons of SMARCB1, multiplex ligation-dependent probe amplification (MLPA), and whole genome single nucleotide polymorphism (SNP) array were performed on frozen tissue from the two adult cases.

Results: Morphologically the tumors were composed of poorly differentiated discohesive cells with eccentric, oval nuclei. Some tumor cells featured abundant eosinophilic inclusion-like cytoplasm, imparting a rhabdoid appearance. All tumors showed cytokeratin reactivity and lack of SMARCB1 expression; no myoid differentiation was identified by IHC. There were no mutations in the promoter region or coding sequence of the SMARCBI gene. One of the two tumors showed heterozygous deletion in chromosome 22 by MLPA and whole genome SNP array.

Conclusions: Though rare, PES could occur in HN and should be differentiated from other HN tumors with epithelioid/epithelial differentiation by lack of SMARCBI staining. Although positive cytokeratin and negative myoid markers favor PES over MRT, molecular analysis may further distinguish these closely related entities. The lack of mutations in the SMARCB1 gene has been reported in PES cases. Although deletion could be seen in both PES and MRT, the finding of heterologous deletion is more common in PES, but rare in MRT, which typically demonstrates homozygous deletions in SMARCB1. The lack of mutation and deletion in a HN PES warrants study to further investigate alternative mechanisms, such as epigenetic silencing, intronic mutations, RNA editing, or post transcriptional control, resulting in loss of SMARCBI expression in PES. 
1299 Evaluation of P63 Autoantibodies as a Biomarker for Head \& Neck Squamous Cell Carcinoma

H Gallai, RO Wein, K Kelsey, LW Solomon. Tufts University, Boston, MA; Tufts Medical Center, Boston, MA; Brown University, Providence, RI; Emory University, Atlanta, GA. Background: Amplification and overexpression of the p63 gene on chromosome $3 q 27-29$ is reported in $85 \%$ of head and neck squamous cell carcinomas (HNSCC) Overexpressed p63 functions as an oncogene. Protein overexpression can override host immune self-tolerance and produce a humoral immune response to self proteins. Previous studies detected serum p63 autoantibodies in $18 \%$ of patients with HNSCC A case of HNSCC was serendipitously found to have p63 overexpression in the tumor cells by immunohistochemistry and serum autoantibodies to $\mathrm{p} 63$ by immunofluorescence studies and enzyme-linked immunosorbent assay (ELISA) (titer $>0.8 \mathrm{OD} /$ controls $<0.1$ OD; OD = optical density 450nm). Our hypothesis is that some patients with HNSCC will have serum autoantibodies to 063 that are detectable by ELISA.

Design: A case control study was designed to test serum from HNSCC patients for the presence of $\mathrm{p} 63$ autoantibodies. A validated ELISA was developed using recombinantly expressed p63 protein (N-terminal aa $1-205$ of the $\Delta \mathrm{Np} 63$ isoform) as the antibody capture agent. nQuery V7.0 software determined the sample size of $100 \mathrm{HNSCC}$ cases and 100 age and sex matched controls $(\alpha=0.05$; power $=99 \%)$. Each serum was independently tested for $\mathrm{IgG}$ and $\operatorname{IgA}$ antibodies. Samples were tested in triplicate and the OD results averaged. SPSS for Windows V16 software was used for all statistical analyses. Receiver operator characteristic (ROC) curves were constructed to determine the optimal cut-off for classifying the disease state; the cut-off value that maximized the Youden Index was selected as the optimal cut-off. An independent-samples t-test was used and p-values less than 0.05 are considered statistically significant. The sensitivity/ specificity and positive/negative predictive values were calculated.

Results: The ELISA test revealed a sensitivity of $54 \%$, specificity of $72 \%$, positive predictive value of $67 \%$ and a negative predictive value of $61 \%$ for IgG antibodies. Testing for IgA antibodies had a sensitivity of $75 \%$, specificity of $47 \%$, positive predictive value of $58.6 \%$ and a negative predictive value of $65 \%$.

Conclusions: Testing for p63 autoantibodies by ELISA is not specific or sensitive enough to be used for screening, prognosis, treatment response monitoring or detection of disease recurrence in HNSCC. Serum p63 autoantibodies are not a reliable biomaker for HNSCC.

\section{Spectrum of Problematic Metaplastic Changes in Salivary Gland} Tumors

DR Gnepp, E Yakirevich, SK El-Mofty. Rhode Island Hospital, Providence, RI; Washington University, St. Louis, MO.

Background: Metaplastic change is a common phenomenon in salivary gland neoplasms. Most of these tumors easily fit into specific diagnostic categories. However, rare tumors present diagnostic challenges due to prominent metaplastic changes. In this study we analyzed the spectrum of salivary gland metaplastic changes causing diagnostic difficulty over a 20 -year period.

Design: 56 salivary gland neoplasms with prominent metaplastic change were retrieved from pathology and consultation files (1990-2010). Ancillary studies (histochemistry, immunohistochemistry \& EM) were applied as necessary.

Results: Patients' age ranged from 17-87 (median 55) years with an equal male: female ratio. 45 cases originated from the parotid, 2 from submandibular and 9 from the minor salivary glands. Metaplastic changes were analyzed separately for epithelial, myoepithelial and stromal components. Epithelial metaplastic changes included, in descending order, squamous, oncocytic, mucoepidermoid, adenoid-cystic, and mucinous metaplasia. Myoepithelial cells demonstrated oncocytic, epithelial-myoepithelial, and adenoid-cystic like changes; the stromal component showed occasional lipomatous metaplasia. $36 \%$ showed more than one type of differentiation. Prominent squamous metaplasia was the most common metaplastic phenomenon and was most frequently associated with pleomorphic adenomas (PA) (14 of 26 cases) and Warthins tumor ( 9 cases); it was also seen in 2 oncocytomas. Diffuse oncocytic metaplasia was a characteristic feature of 20 tumors, including 8 PA, 5 acinic cell carcinomas, 2 mucoepidermoid carcinomas, 2 myoepitheliomas, 2 cystadenomas \& 1 epithelialmyoepithelial carcinoma. Less frequent metaplastic changes included prominent mucoepidermoid (3), adenoid-cystic (3), mucinous (2), \& epithelial-myoepithelia (1) differentiation. All the less frequent changes were seen exclusively in PA. Careful sampling and light microscopy looking for absence of destructive overgrowth of benign elements, lack of perineural, vascular, or extracapsular invasion \& bland nuclear features will support a diagnosis of a benign metaplastic salivary gland tumor. Recognizing foci of residual classical appearing tumor in addition to the more prominent metaplastic component will allow proper classification.

Conclusions: Prominent metaplastic changes, although rare, may occasionally obscure the true nature of a salivary gland neoplasm. Recognizing the spectrum of metaplastic change will aid in establishing proper diagnoses and treatment.

\section{FGFR1 Amplification in Metastatic Squamous Cell Carcinoma of} the Head and Neck - A Potential Target for a Rational Therapy?

F Goeke, A Franzen, R Mennon, R Kirsten, D Boehm, W Vogel, F Bootz, A Schroeck, $S$ Perner. University Hospital Bonn, Bonn, Germany.

Background: Currently, patients with FGFR1 amplified squamous cell lung cancers (L-SCC) are treated in phase I clinical trials using small molecule inhibitors. Of interest, SCC of the lung share common molecular alterations with squamous cell head and neck cancers (HN-SCC). Aim of our study is to assess if HN-SCCs also harbor FGFR1 amplifications.
Design: We put together a cohort of 227 patients suffering from HN-SCC, with 97 of these suffering from metastatic disease. Primary tumors and, where available, metastatic tumors were assessed for FGFR1 copy number status using fluorescence in-situ hybridization (FISH)

Results: $20,3 \%$ of primary HN-SCC displayed FGFR1 amplifications. Of interest, almost all metastatic tumor samples revealed a FGFR1 amplification if the corresponding primary tumor harbored the amplification.

Conclusions: FGFR1 amplification frequently occurs in primary and metastatic $\mathrm{HN}$ SCC and proves as a potential target for small molecule therapy in non-operable or metastatic disease. Further functional studies and subsequent clinical trials are needed for further validation of our findings.

1302 SOX2 Amplification Is a Common Event in Sinunasal Squamous Cell and Undifferentiated Carcinomas

F Goeke, A Franzen, R Mennon, S Huss, D Boehm, W Vogel, F Bootz, A Schroeck, $S$ Perner. University Hospital Bonn, Bonn, Germany; University Hospital Cologne, Cologne, Germany.

Background: Although carcinomas of the nasal cavities are known to differ significantly from other cancers of the head and neck regarding causing noxa, clinical behavior and treatment, they share histological appearance. SOX2, a transcription factor-coding gene located at 3q26.33, is known to be recurrently amplified in squamous cell carcinomas (SCCs) of the lung, esophagus, skin, penis, cervix uteri and oral cavity. The aim of our study was to assess if SOX2 amplifications also occur in different tumor entities of the paranasal sinuses.

Design: Using flourescence in-situ hybridization, we assessed for SOX2 amplification status in a cohort consisting of sinonasal SCCs $(n=65)$, sinonasal undifferentiated carcinomas (SNUC, $n=18$ ), adenocarcinomas $(n=25)$, and adenoid cystic carcinomas $(\mathrm{n}=18)$. Furthermore, we performed SOX2 immunohistochemical staining to quantify protein expression.

Results: We detected SOX 2 amplifications in $36 \%$ of sinonasal SCCs, $35 \%$ of SNUCs, $9 \%$ of adenocarcinomas, but none of the adenoid cystic carcinomas. Moreover, we found that the SOX2 amplification is associated with a SOX2 protein overexpression in SCCs and SNUCs.

Conclusions: SOX2 amplification is not an organ site specific event, but is a frequent genetic alteration occuring in SCCs of various organs. Since SNUCs also harbor SOX2 amplifications in similar frequencies, we hypothesize that SNUCs may be undifferentiated SCCs.

1303 Mammary Analogue Secretory Carcinoma: Expansion of a Clinicopathologic Profile and Identification of New Sources for Recategorization

CC Griffith, S Chiosea, RR Seethala. UPMC, Pittsburgh, PA.

Background: Mammary analogue secretory carcinoma (MASC) of salivary glands is a recently described tumor, histologically and biologically similar to secretory carcinoma of breast (ETV6-NRTK3 translocated). It is assumed that most were historically classified as acinic cell carcinoma (AciCC) but other 'reservoirs' may exist. In order to expand and refine the clinicopathologic profile of this rare tumor, we combine prospectively diagnosed MASC with a retrospective cohort derived from an array of diagnostic categories.

Design: MASC were prospectively diagnosed over a 10 month period. Also, 297 archived salivary gland tumors, spanning several diagnostic categories, were reviewed by histomorphology and immunophenotype. Cases with pink vacuolated cytoplasm, luminal mucin, and/or strong S100 positivity were evaluated for ETV6 translocation by FISH ( $\mathrm{n}=67$ ). Clinicopathologic parameters on confirmed cases were recorded. Reservoirs of MASC

\begin{tabular}{|l|l|l||}
\hline Original diagnosis & Number reviewed & Number reclassified as MASC \\
\hline Adenocarcinoma, NOS & 24 & 14 \\
\hline AciCC & 91 & 11 \\
\hline Mucoepidermoid carcinoma & 165 & 1 \\
\hline Signet ring cell carcinoma & 5 & 1 \\
\hline Polymorphous low grade adenocarcinoma & 4 & 0 \\
\hline Sinonasal non-ITAC* & 4 \\
\hline Other & 4 \\
\hline
\end{tabular}

*non-ITAC $=$ non-intestinal type adenocarcinoma

Results: A total of 36 MASC were confirmed ( 9 prospective and 27 retrospective). Sources of retrospectively identified MASC are summarized in the table. 30 case were drawn from our consult files. Tumor sites were 26 parotid, 3 submandibular, 3 soft palate, 1 buccal mucosa, 1 upper lip and 1 base of tongue. MASC occurred in a wide age range (10-75 years, average 45.6 ) and had a male predominance ( 21 male: 14 female). 15 tumors were uninodular/cystic, 13 infiltrative and 4 multinodular/cystic. 18 tumors showed predominantly cribriform architecture, 9 solid, 5 intracystic and 4 papillary. 19 showed at least rare papillae and 22 at least some intracystic growth. Perineural and angiolymphatic invasion was common, $36.7 \%$ and $20 \%$ respectively. All tested cases were $\mathrm{S} 100$ positive $(\mathrm{n}=25)$ but half showed variable to focal staining. p63 showed peripheral staining in $5 / 22$ cases, thus highlighting intraductal components. 5 cases were clinically aggressive - 4 nodal metastasis and 1 disease related death following recurrence and metastasis.

Conclusions: A wide array of diagnostic categories should be surveyed to more accurately characterize MASC, since in addition to AciCC, adenocarcinoma, NOS is a common source of MASC. MASC demonstrate a variety of morphologic features and range from low to high grade. Papillary and intracystic growth are fairly common. A subset of tumors behave aggressively. 
1304 Inverse Relationship between HPV-Infection and EGFR Gene Copy Number in Head and Neck Squamous Cell Carcinomas

$S$ Hakim, S Moyano, E Bailon, A Valera, A Nadal, I Alobid, JJ Grau, L Alos. Hospital Clinic, Barcelona, Spain.

Background: HPV-related squamous cell carcinomas of the head and neck are more prevalent in oropharynx and sinonasal tract. The better prognosis of these tumors when comparing with HPV-negative tumors has been repeteadly reported. The EGFR gene encodes a protein that can be activated by phosphorylation, inducing a signalling transduction cascade which ultimate in cell proliferation and survival. The EGFR overexpression has been linked to a bad prognosis in several cancers, including the head and neck carcinomas.

Design: The aim of the study was to stablish the relationship between the HPV-infection and the EGFR gene status in a series of head and neck squamous cell carcinomas.

Eighty eight patients with squamous cell carcinoma of the head and neck (50 sinonasal and 38 oropharyngeal) were included in the survey. HPV was detected by PCR using SPF10 primer set (Innogenetics Diagnostica, Barcelona, Spain). EGFR gene was studied by FISH using dual-colour EGFR Spectrum-red/CEP7 Spectrum-green probe (Dako, Carpinteria, CA, USA). Immunohistochemistry for P16 ${ }^{\mathrm{INK} 4}$ (Biocare Medical, Walnut Creek, CA, USA; Clone JC8; dilution 1:100) and EGFR (Dako; dilution 1:100) proteins was performed.

Results: Tumors affected 69 males and 19 females with a median age of 62 (range $25-93$ ) years. HPV16 was detected in 18 cases ( $20 \%$ of tumours). The HPV- positive carcinomas affected more frequently females $(53 \%$ of patients $)(p<0.0004)$, but no significant differences in age were found. $\mathrm{P} 16^{\mathrm{INK} 4}$ positivity correlated with HPVdetection in $100 \%$ of cases. High EGFR gene copy number was detected in $85 \%$ of HPV-negative carcinomas ( 5 amplifications and 55 polysomies); and in $39 \%$ of HPVpositive carcinomas ( 7 EGFR polysomies) $(\mathrm{p}<0.0001)$. No EGFR amplifications were detected in HPV- positive carcinomas. No correlation between EGFR gene status and EGFR protein expression was observed.

Conclusions: The molecular differences between HPV-positive and HPV-negative squamous cell carcinomas of the head and neck include the EGFR-gene status. This result may have great relevance to tailor oncological treatments in these patients.

\section{Fibrosing Variant of Hashimoto's Thyroiditis Is an IgG4 Related} Disease

AE Huck, WC Faquin, JH Stone, GP Nielsen, V Deshpande. Massachusetts General Hospital and Harvard Medical School, Boston, MA.

Background: Hashimoto's thyroiditis (HT) and the fibrosing variant of Hashimoto's thyroiditis (FVHT) are immune-mediated tumefactive lesions of the thyroid. IgG4related disease (IgG4-RD) is now a widely recognized multi-organ system disease characterized by elevated serum and tissue concentrations of IgG4. The sine qua non of IgG4-RD is its histologic appearance: lymphoplasmacytic inflammation, storiform fibrosis, and obliterative phlebitis. Recent data from Japan suggest that a subset of patients with HT also belong to the IgG4-RD spectrum. We address several questions pertaining to the relationship among HT and FVHT and the association of each of these diseases with IgG4-RD.

Design: We evaluated 28 consecutive cases of HT and 9 cases of FVHT. The clinical, demographic, and serological data were recorded. The slides were stained immunohistochemically using antibodies to IgG4 and $\mathrm{IgG}$, and the degrees of plasma cell infiltration and IgG4 staining within samples were quantified. For each case, the number of plasma cells staining for IgG4 and IgG was assessed in non-overlapping high-power fields.

Results: Hypothyroidism was noted in $62 \%$ of HT and $85 \%$ of FVHT. Thyroid peroxidase antibodies were positive in all 10 cases of HT and all 4 cases of FVHT. FVHT demonstrated an exaggerated lobular pattern with lobules separated by storiform type fibrosis. This histological appearance was remarkably similar to the interlobular stroma of IgG4-associated sialadenitis and the type 1 variant of autoimmune pancreatitis. Follicular cells were predominantly arranged in a microfollicular pattern, and an intense inflammatory infiltrate composed of lymphocytes and plasma cells was identified between follicles. The mean IgG4 counts per HPF in HT and FVHT were 4.9 and 27, respectively. The mean IgG4:IgG ratios in HT and FVHT were $19.0 \%$ and $61.4 \%$, respectively.

Quantitation of IgG4 and IgG in Hashimoto's thyroiditis, and fibrosing variant of Hashimoto's thyroiditis.

\begin{tabular}{|l||l|l|l||}
\hline & Mean IgG4 per HPF & Mean IgG per HPF & Mean IgG4/IgG ratio \\
\hline \hline Hashimoto's thyroiditis & $4.9(8.1)$ & $22.7(12.6)$ & $19 \%(22.7)$ \\
\hline Fibrosing variant of & $27.0(17.9)$ & $43.5(19.5)$ & $61.4 \%(30.6)$ \\
\hline Hashimoto's thyroiditis & $\mathrm{p}<0.0001$ & $\mathrm{p}<0.0001$ & $\mathrm{p}<0.0001$ \\
\hline P value & & \\
\hline
\end{tabular}

Figures in parenthesis represent Standard deviation.

Conclusions: The dense lymphoplasmacytic infiltrate and interlobular fibrosis along with elevated numbers of IgG4 positive plasma cells link this disease to IgG4-RD. We propose that FVHT belongs to the IgG4-RD spectrum.

1306 WNT-ß-Catenin Is Upregulated in Very Aggressive Tumor Pattern of Invasion

T Isayeva, M Brandwein-Gensler. UAB, Birmingham, AL

Background: The WNT pathway is a critical to proliferation, differentiation, oncogenesis and is upregulated in many cancers. Among the downstream effects, WNT- $\beta$-catenin is involved in tumor invasion. We developed 4 cell lines from oral/ oropharyngeal SCC, which are unique as they are matched to different worst patterns of invasion (WPOI) in the corresponding resections. These histopathological WPOI were developed and validated by us as outcome prognosticators.

Design: Cell lines were derived from fresh resections, cancer cells were sorted from cancer-associated fibroblasts by flow cytometry and grown in keratinocyte media/ keratinocyte growth supplements. HPV was determined by RT-PCR with HPV16/18 E6/E7 primers, with appropriate controls. Invasion was measured with the BioCoat Matrigel chamber, $24 \mathrm{~h}$ after stimulation with TGFß. Cells that invaded across the chamber membrane were counted after fixation and staining. UAB-1, UAB-4, and the corresponding frozen samples from the 2 resections were assessed for WNT signalling with the RT2 96 well RT-PCR kit (SABiosciences)

Results: Table 1 shows the clinicopathologic features for these cells. Table 1: UAB Cell Lines

\begin{tabular}{|l|l||l|l||l||}
\hline \hline & UAB-1 & UAB-2 & UAB-3 & UAB-4 \\
\hline Pattern of invasion, corresponding resection & WPOI3 & WPOI3 & WPOI5 & WPOI5 \\
\hline HPV16/18 & Pos/Neg & Neg/Neg & Neg/Neg & Neg/Neg \\
\hline Site & Orophx & Oral & Oral & Oral \\
\hline Invasion Assay & 3.0 & 14.0 & 32.7 & 40.7 \\
\hline
\end{tabular}

Mean invading tumor cell counts after TGF $\beta$ stimulation, normalized for control (no stimulation)

UAB-1 was HPV16+/HPV18-, all other cells were HPV. Transwell assays showed increased invasion in WPOI5 cells compared with WPOI3 cells after stimulation.

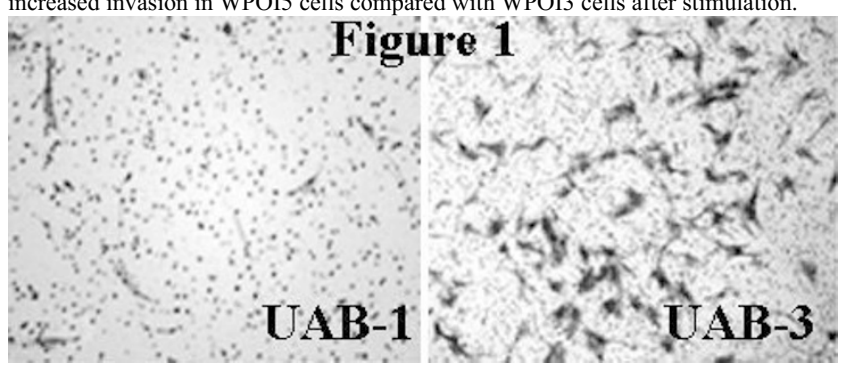

RT-PCR results shows up-regulation of WNT-pathway in tumor-2/UAB-4, compared to tumor-1/UAB-1.

Table 2: WNT in UAB-1 \& UAB-4
\begin{tabular}{||l|l|l|l|l||}
\hline $\begin{array}{l}\text { Gene } \\
\text { WISP1 }\end{array}$ & $\begin{array}{l}\text { Tumor-1 } \\
\text { WPOI3) }\end{array}$ & $\begin{array}{l}\text { UAB-1 derived } \\
\text { from Tumor-1 }\end{array}$ & $\begin{array}{l}\text { Tumor-2 (WPOI5) } \\
\text { WISA-4 derived from Tumor-2 }\end{array}$ & UAB \\
\hline WNT1 & 0 & 4.2 & 1.6 & 6.7 \\
\hline WNT6 & 1.4 & 5.9 & 5.1 & 15.2 \\
\hline WNT7A & 0 & 3.6 & 4.9 & 39.1 \\
\hline WNT10A & 0 & 0 & 4.9 & 14.9 \\
\hline WNT11 & 0 & 2.5 & 5.9 & 35.8 \\
\hline CTNNB1 & 0 & 6.5 & 3.5 & 9.8 \\
\hline
\end{tabular}

RT-PCR, $\mathrm{C}(\mathrm{t})$ normalized for mean of 5 internal controls

Conclusions: Cell lines derived from WPOI5 carcinomas (aggressive pattern of invasion) have greater invasive characteristics, compared to cell lines from WPOI3 (nonaggressive) tumors. Aggressive tumor invasion is associated with upregulated WNT signalling. These cell lines will serve as a mechanistic model of invasion in ongoing studies.

1307 Increased Expression of Cytokeratin 19 in Oral Squamous Cell Dysplasia Is Associated with Susceptibility to Infection with Epstein-Barr Virus (EBV)

R Jiang, X Gu, T Moore-Medlin, C-A Nathan, L Hutt-Fletcher. LSUHSC, Shreveport, LA.

Background: EBV, an orally transmitted herpesvirus that targets B cells and epithelial cells, is associated with development of both B cell lymphomas and nasopharyngeal carcinoma. Infection of B cells is initiated by binding of virus to the complement receptor CD21. Infection of CD21-negative epithelial cells is inefficient but efficient infection can be achieved if expression of CD21 is engineered. Whether EBV infection plays an etiologic role in oral squamous cell dysplasia (OSD) and malignant transformation is unclear. The aim of this study was to evaluate CD21 expression in OSD and its association with the epithelial stem cell marker CK19 that is elevated in oral squamous cell carcinoma (OSCC)

Design: Formalin-fixed paraffin-embedded tissue blocks were retrieved and separated by pathologic grade as follows: normal margin epithelium of OSD $(n=6)$, low-grade OSD (LSD, $n=8$ ) and high-grade OSD (HSD, $n=21$ ). Squamous cells were isolated by laser capture microdissection. Isolated mRNA was amplified using qRT-PCR with target primers specific for the CD21 exons coding for the EBV binding site, for CK19 and for EBV small RNA, EBER1. CK19 expression was also examined by immunohistochemistry (IHC). IHC for CD21 has not proven reliable.

Results: Normal margins of OSD were negative for both CD21 and EBV. CD21 expression was found in $12.5 \%(1 / 8)$ LSD samples and $57.1 \%(12 / 21)$ HSD samples. Transcript levels were 0.35 and 2.97 fold higher, respectively, than in the Akata B cell line (set arbitrarily at 1). EBV could also be detected in some samples of OSD. CK19 was detected in normal epithelial margin at low transcript levels. Transcript levels were increased significantly in EBV positive OSD $(\mathrm{P}<0.025)$, but not in EBV negative OSD ( $>0.05$ ). Increased CK19 was confirmed at the protein level by IHC.

Conclusions: CD21 mRNA is expressed in dysplastic oral epithelium, and is upregulated as the grade of dysplasia increases. Expression of CK19 is increased in premalignant dysplasia and the increase is correlated with the presence of EBV. CK19 is a stem cell marker which is elevated in OSCC and is associated with increased epithelial mesenchymal transition. Increased susceptibility of epithelial cells to EBV may be involved in the process of oral squamous cell differentiation and precipitate malignant transformation. 
1308 Differential Expression of CyclinD1 and p16 ${ }^{\mathrm{INK} 4 \mathrm{~A}}$ in Benign and Malignant Salivary Gland Tumors: A Study of 44 Cases

$G$ Jour, $K$ West, V Ghali, G Ephrem, B Wenig. BIMC, New York, NY.

Background: Salivary gland tumors comprise a morphologically and biologically diverse group of lesions. There is conflicting data concerning the molecular events involving the $\mathrm{pRb}$ pathway in these tumors.Recent studies suggest that promote hypermethylation or homozygous deletion of $\mathrm{p} 16^{\mathrm{INK} 4 \mathrm{a}}$ gene in adenoid cystic carcinomas(AdCC) and mucoepidermoid carcinomas (MEC) are the most common mechanisms for $16^{\mathrm{INK} 4 \mathrm{~A}}$ silencing. Few studies examined the alterations in the $\mathrm{Rb}$ pathway by immunohistochemical (IHC) methods in benign and malignant salivary gland tumors. Furthermore, recent evidence implicates HPV in MEC carcinogenesis The purpose of our study is to examine $\mathrm{p} 16^{\mathrm{INK} 4 \mathrm{~A}}$ and cyclin D1 expression in a variety of benign and malignant salivary gland tumors, and to investigate p16 expression as a surrogate marker for HPV infection in MEC.

Design: 44 cases were selected from the Pathology files in our hospital centers. The series includes 31 malignant tumors (14 MEC, 5 acinic cell carcinomas (ACC), 5 polymorphous low grade adenocarcinomas (PLGA), 6 (AdCC) and 14 benign tumors ( 4 benign cysts, 5 Warthin tumors and 5 pleomorphic adenomas (PA). All cases were tested by IHC for $16^{\mathrm{INK} 4 \mathrm{~A}}$ and Cyclin D1. Testing for HPV wide spectrum (HPV-WS) was performed by in situ hybridization in all MEC cases. Staining intensity was recorded semi quantitatively (on a scale from 0 to $4+$ ). Fisher's exact test and correlation with a $\mathrm{p}<0.05$ were used to assess the differences and correlation in frequencies of various parameters tested.

Results: Cyclin D1 and p16 are expressed similarly in malignant and benign tumors ( $p=0.126$ and $p=0.464$, respectively). None of the MEC cases showed nuclear reactivity for HPV-WS. Statistical analysis showed positive correlation between Cyclin D1 and p16 expression.

Conclusions: 1) CyclinD1 and $\mathrm{p} 16^{\mathrm{INK} 4 \mathrm{~A}}$ are overexpressed in both malignant and benign salivary gland tumors but not within the normal salivary gland epithelium; the lack of statistical difference between the two groups suggests that these deregulations are unrelated to their biological behavior; 2) The absence of nuclear reactivity for HPVWS in all MEC cases suggests that p16 overexpression in these tumors cannot be used as a surrogate marker for viral infection; 3) The positive correlation between CCND1 and $\mathrm{p} 16^{\mathrm{INK} 4 \mathrm{~A}}$ suggests that CyclinD1 gene over expression may represent a priamry tumorigenic event in salivary gland neoplasms leading to secondary $\mathrm{p} 16^{\mathrm{INK} 4 \mathrm{~A}}$ over expression by an ineffective positive feedback mechanism, similar to that seen in HPV infection. Further molecular studies targeting the CyclinD1 gene locus are warranted.

\section{Prognostic Features and Grading in Mucoepidermoid Carcinoma} of Major Salivary Glands

N Katabi, R Ghossein, S Ali, S Dogan, D Klimstra, I Ganly. MSKCC, New York. Background: Mucoepidermoid carcinoma (MEC) has widely diverse biologic behavior Grading MEC is important prognostically and therapeutically; however, there is no uniformly accepted grading system. The aim of this study is to assess adverse prognostic features of MEC in relation to outcome and to compare different grading systems. Design: 72 cases of MEC were subjected to a detailed histopathologic analysis. Four grading systems were used: AFIP, Brandwein, Healy, and our own (personal system mainly based on the architecture and cytology of the tumor). $5 \mathrm{yr}$ estimates of survival were performed.

Results: 20 of 72 cases were misdiagnosed as MEC; 4 as low grade (LG) and 18 as high grade (HG). The $18 \mathrm{HG}$ were reclassified as follows: 2 salivary duct carcinoma, 11 squamous cell carcinoma, and $3 \mathrm{HG}$ carcinoma unclassifiable. Among the $52 \mathrm{MEC}$, median patient age was 50 with female predilection of $1: 2$. Tumors were predominantly located in the parotid (50 parotid/2 submandibular). Mean tumor size was $1.77 \mathrm{~cm}$ Lymphoid stroma was found in $83 \%$ of tumors. Perineural invasion, vascular invasion, and lymph node metastasis were identified in $19 \%, 6 \%$, and $9 \%$, respectively. Only 1 patient developed distant metastasis to the lung. Median follow-up was 50 month (1-237). $5 \mathrm{yr}$ estimates of disease specific survival (DSS) and recurrence free survival (RFS) were $95 \%$ and $89 \%$, respectively. Mitosis ( $\geq 4 / 10 \mathrm{HPFs}$ ), necrosis, pleomorphism, focal keratinization, desmoplasia, and lymph node metastasis were associated with adverse DSS and RFS $(\mathrm{P}<.002)$. Vascular invasion, tumor size $>2 \mathrm{~cm}$, and infiltrative borders were associated with adverse RFS $(\mathrm{P}<.002)$. In all 4 grading systems, $\mathrm{LG}$ and intermediate grade (IG) had similar DSS and RFS survival but much better outcome than $\mathrm{HG}(\mathrm{P}<0.007)$. All patients with a low mitotic rate and no necrosis did not recur except for 1 patient with a positive margin. All patients with tumors showing a high mitotic rate and necrosis died or recurred. Lack of consensus among grading systems was found in 23 out of 52 cases. Among the 23 cases, 3 LG tumors and 1 IG using AFIP criteria were upgraded to $\mathrm{HG}$ using Brandwein. None of these 4 patients died or recurred. Conclusions: 1) HG MEC is very rare and the majority of previously diagnosed HG MEC cases are misclassified. 2) There is no difference in outcome between LG and IG using any grading system. 3) Consideration should be given to stratify MEC into two grade categories based on relatively objective criteria such as mitosis sand necrosis.

1310 Does p16 Positive Squamous Cell Carcinomas of the Larynx Correlate with HPV? An Analysis of 101 Cases Using E6/E7 mRNA HPV H7 RNA Scope, HPV-16 and High Risk HPV Chromogenic In Situ Hybridization SM Kirby, T Teknos, A Agrawal, M Old, E Ozer, H Iwenofu. OSUMC, Columbus. Background: The significance of human papillomavirus (HPV) in oropharyngeal squamous cell carcinoma $(\mathrm{SqCC})$ is well established. However, $\mathrm{HPV}$ oncogenesis in the larynx is less clear. p16 expression is strongly associated with HPV in oropharyngeal $\mathrm{SqCC}$ and its utility as a surrogate marker has been validated. Implications of laryngeal SqCC p16 expression are less well described. Recently, a specific HPV test has been developed; chromogenic in situ hybridization (ISH) to detect mRNA of the E6/
E7 oncogenes, which are directly involved in dysregulating the cell cycle in HPV oncogenesis. Herein, we examined laryngeal $\mathrm{SqCC}$ using p16 immunohistochemistry (IHC), chromogenic ISH for HPV-16, high risk (HR) HPV DNA and E6/E7 mRNA HPV H7 RNA scope.

Design: A computerized search yielded $101 \mathrm{SqCC}$ of the larynx at OSUMC, which were classified as supraglottic, glottic, or subglottic. Tumor sections were used to construct tissue microarrays (TMA). The TMAs were stained for $\mathrm{p} 16$ using routine IHC methods and evaluated for intensity (0-3+) and percent of tumor cells stained. Staining of $<5 \%$ was considered negative. The TMAs were also stained using ISH for HPV-16 and HR-HPV DNA as well as E6/ E7 mRNA H7 $(16,18,31,33,35,52,58)$, which were interpreted as positive or negative.

Results: p16 expression was present in 20/101 (18\%) laryngeal SqCC. Supraglottic SqCC showed rate of $30 \%$ (14/46) including 8 with strong and diffuse staining (3+, $>75 \%)$. Glottic SqCCs had a positive rate of $8 \%(4 / 52)$ with weaker staining. 1/9 (11\%) subglottic SqCC was positive with only focal staining. The difference in 16 expression for supraglottic, glottic and subglottic groups were statistically significant $(\mathrm{p}<0.05)$ All tumors were negative for HPV-16 and HR-HPV ISH. HPV E6/E7 mRNA H7 was detected in $5 \%(1 / 20)$ of p16 positive tumors. This tumor was supraglottic with strong and diffuse $\mathrm{p} 16$ expression.

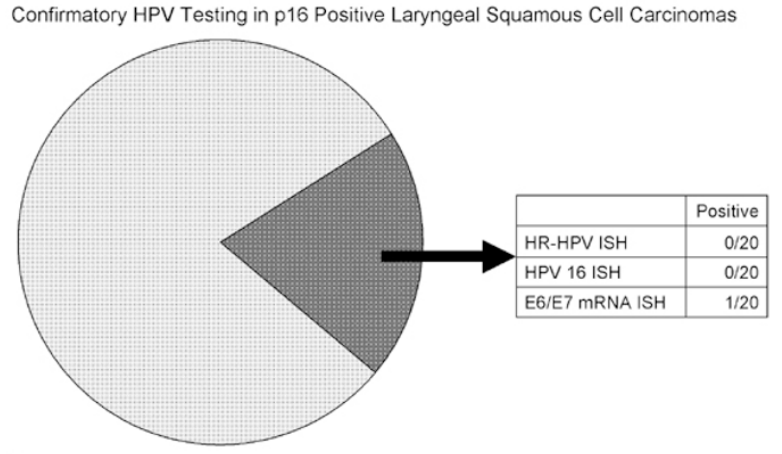

$\square$ p16 Negative $\mathbf{0}$ p16 Positive

Conclusions: Our study indicates a low overall frequency rate of $18 \%$ p 16 positivity in SqCC of the larynx with predilection for supraglottic sites. Only $5 \%$ of $\mathrm{p} 16$ positive cases harbour HPV using a highly sensitive assay. Our data seems to suggest that p16 expression does not correlate with HPV infection in the larynx and might be unrelated to HPV oncogenesis.

1311 p16 Expression and Prognostic Value in Laryngeal Squamous Cell Carcinomas - A Large Cohort Study from Chinese Patients

$J$ Leidy, K Chen, T Stockl, W Ye, Q Wu, J Liang, B Woda, Z Jiang. University of Massachusetts Medical School, Worcester, MA; State Key Laboratory of Oncology in South China, Guangzhou, Guangdong, China; SunYat-sen University, Guangzhou, Guangdong, China.

Background: In the United States and Europe, about $40-80 \%$ of head and neck squamous cell carcinoma are caused by human papillomavirus (HPV). p16 expression and HPV infection have been demonstrated to be a favorable biomarker for patients with head and neck squamous cell carcinoma. However, p16 expression and its prognostic value of laryngeal squamous cell carcinoma in patients from China are unknown. We aimed to investigate p16 expression and its prognostic value in Chinese patients with laryngeal squamous cell carcinoma.

Design: A total of 236 patients with laryngeal squamous cell carcinomas (laryngectomy, $\mathrm{n}=111$; biopsy, $\mathrm{n}=127$ ) obtained from the Surgical Pathology file (2000-2005), Cancer Center of SunYat-sen University, Guangzhou, People's Republic of China were examined by immunohistochemistry for $\mathrm{p} 16$ expression. Overall survival was measured from the date of laryngectomy or biopsy to the date of death, and was censored from the date of last follow-up. Median follow-up period was 5 years and 3 months (63 months, range 7-123 months).

Results: Table 1 provides the relevant clinical characteristics of the 236 patients with laryngeal squamous cell carcinoma and p16 expression. Only 43 of $236(18 \%)$ laryngeal carcinomas expressed p16. Age, sex, tumor stage and grade were not associated with p16-positive status. 
Clinicopathological characteristics of patients with laryngeal carcinoma

\begin{tabular}{|c|c|c|c|}
\hline & p16-Negative & p16-Postitive & P Value \\
\hline & $\mathrm{n}=193$ & $\mathrm{n}=43$ & \\
\hline \multicolumn{4}{|l|}{ Sex } \\
\hline Female & $4(50 \%)$ & $4(50 \%)$ & 0.04 \\
\hline Male & $189(83 \%)$ & $39(17 \%)$ & \\
\hline \multicolumn{4}{|l|}{ Age } \\
\hline Mean (SD) & $58(9.9)$ & $59(10.8)$ & 0.57 \\
\hline \multicolumn{4}{|l|}{ Stage } \\
\hline I & $58(81 \%)$ & $14(19 \%)$ & 0.77 \\
\hline III & $59(86 \%)$ & $10(14 \%)$ & \\
\hline III & $52(81 \%)$ & $12(19 \%)$ & \\
\hline IV & $24(77 \%)$ & $7(23 \%)$ & \\
\hline \multicolumn{4}{|l|}{ Grade } \\
\hline In situ & $10(91 \%)$ & $1(9 \%)$ & 0.39 \\
\hline W & $75(86 \%)$ & $12(14 \%)$ & \\
\hline M & $79(79 \%)$ & $21(21 \%)$ & \\
\hline $\mathrm{P}$ & $29(76 \%)$ & $9(24 \%)$ & \\
\hline \multicolumn{4}{|l|}{ Vital Status } \\
\hline Dead & $64(83 \%)$ & $13(17 \%)$ & 0.71 \\
\hline Alive & $129(81 \%)$ & $30(19 \%)$ & \\
\hline
\end{tabular}

Kaplan-Meier analysis showed that the 10 -year rate of overall survival was similar in patients with p16 positive tumors and patients with p 16 negative tumors $(31 \%$ vs. $34 \%$; $\mathrm{P}=0.28 ; 95 \%$ confidence interval $[\mathrm{CI}], 0.34$ to 1.37 ).

Conclusions: p16 expression was only found in $18 \%$ Chinese patients with laryngeal carcinomas. Unlike Caucasian patients, this large cohort study provides the first evidence that HPV positivity is not a favorable biomarker for patients with laryngeal squamous cell carcinomas in China.

1312 Partial p16 Immunoreactivity in Oropharyngeal Squamous Cell Carcinoma - Extent and Pattern of Staining Correlate with the Presence of Transcriptionally-Active Human Papillomavirus

JS Lewis, RD Chernock, X-J Ma, JJ Flanagan, Y Luo, X Wang, T Zhang, WL Thorstad, SK El-Mofty. Washington University, St. Louis, MO; Advanced Cell Diagnostics, Inc., Hayward, CA.

Background: Human papillomavirus (HPV)-related oropharyngeal squamous cell carcinoma (SCC) is a tumor with unique biology and better outcomes. p16 immunostaining is extensively used as a surrogate marker for transcriptionally-active HPV. While diffuse staining is generally accepted as being positive, the significance of partial staining has not been established, nor has the cutoff for extent of p16 staining that should be used to identify a tumor as HPV-related or unrelated.

Design: From 3 other large studies ( $>400$ cases) utilizing p16 immunohistochemistry, we identified all cases with partial positive staining. The p16 stained slides were reviewed by 3 study pathologists for staining (both nuclear and cytoplasmic) extent (in quartiles) and also for $\%$ of staining that was confluent (i.e. back to back positive cell staining). Tumors were histologically typed (keratinizing, nonkeratinizing, or nonkeratinizing with maturation) and tested for high risk HPV by RNA in situ hybridization (ISH) and reverse transcriptase PCR.

Results: For the 16 cases, there were $24+(13 \%), 53+(31 \%), 62+(38 \%)$, and $31+$ $(19 \%)$ p16 staining tumors. Extent of staining ranged from 5 to $90 \%$ of cells positive with $25 \%$ or more confluent staining in $4 / 16(25 \%)$. Table 1 shows histology, HPV testing, and 16 results by quartile.

Table 1 - HPV Testing by 16 Staining Extent

\begin{tabular}{|c|c|c|c|c|c|}
\hline p16 & HPV RNA ISH & HPV RTPCR & Nonkeratinizing & $\begin{array}{l}\text { p16 Staining } \\
\text { (avg \%) }\end{array}$ & $\begin{array}{l}\text { p16 Confluent } \\
\text { Staining }(25 \% \\
\text { cutoff) }\end{array}$ \\
\hline $1+(0-25 \%)$ & $0 / 3$ & $1 / 3$ & $0 / 3$ & 12 & $0 / 3$ \\
\hline $2+(26-50 \%)$ & $0 / 6$ & $0 / 5$ & $0 / 6$ & 35 & $0 / 6$ \\
\hline $3+(51-75 \%)$ & $3 / 5$ & $2 / 4$ & $2 / 5$ & 61 & $3 / 5$ \\
\hline $4+(76-100 \%)$ & $2 / 2$ & $2 / 2$ & $2 / 2$ & 85 & $1 / 2$ \\
\hline
\end{tabular}

Five of $16(31 \%)$ cases were HPV-related based on RNA ISH and RT-PCR. All of these cases had $>50 \%$ staining, $4 / 5(80 \%)$ had more than $25 \%$ confluent staining, and $4 / 7$ $(57 \%)$ had nonkeratinizing histologic features. Only 1 of the p16 1+/2+ tumors was HPV RNA positive (by RTPCR only and low level). All $1+/ 2+$ cases were keratinizing type or undifferentiated.

Conclusions: By sensitive detection methods, most partial p16 positive SCC cases with $>50 \%$ staining harbor transcriptionally-active HPV, and most HPV+ tumors have significant amounts of confluent staining. Cases with $<50 \%$ p16 staining and lacking significant confluent staining rarely harbor HPV. Thus, our study suggests that $>75 \%$ p16 staining or, alternatively, $>50 \%$ staining combined with $>25 \%$ confluent areas, are suitable criteria for use for positivity for $\mathrm{p} 16$ in OSCC.

1313 Grading of Head-Neck Squamous Dysplasia - A Multi-Insitutional Study

H Mani, M Richardson, R Seethala, J Lewis, J Hunt, E Schaefer, H Crist. PSMSHMC, Hershey; MUSC, Charleston; UPMC, Pittsburgh; WUSTL, St Louis; UAMS, Little Rock.

Background: Grading of squamous dysplasia on head-neck mucosal biopsies plays a central role in management, but is subjective. None of the limited studies on observer variation in grading has included community pathologists. This study compares observer variation in grading between subspecialized head-neck pathologists (HNP) and general surgical pathologists (GSP). This is the first study employing digital imaging as a media for such evaluation at this site.

Design: Aperio scanned images of a random selection of 50 head-neck mucosal biopsies were circulated to 7 HNP ( 4 surgical and 3 oral) and 7 GSP ( 3 academic and 4 community). Participants were asked to assign 3-tier (negative, mild, moderate, severe) and 2-tier (negative, low, high) grades on each case. Results were analyzed for observer agreement using Krippendorf alpha (KA), a measure more appropriate than kappa statistics for ordinal data. 5 cases rated suboptimal were excluded.

Results: Percent cases graded as no/mild/moderate/severe dysplasia were 18/18/19/45 by HNP and $20 / 26 / 18 / 36$ by GSP. HNP graded $18 / 27 / 55$ cases as no/low/high grade dysplasia compared to $21 / 33 / 46$ by GSP. Severe/high grades were assigned more often by oral HNP (54/61\%) than by surgical HNP (38/51\%), academic GSP $(46 / 54 \%)$ and community GSP $(28 / 40 \%)$. HNP made a diagnosis of moderate dysplasia in 61 instances, of which $29(48 \%)$ were classified as low and $32(52 \%)$ as high grade. GSP made a diagnosis of moderate dysplasia in 56 instances, of which $23(41 \%)$ were classified as low and $33(59 \%)$ as high grade. On 3-tier grading, agreement was only moderate among all pathologists (KA 0.56), and was better among GSP (0.62) than $\operatorname{HNP}(0.56)$. 2 -tier grading resulted in less agreement with KA values being 0.48 (all pathologists), 0.47 (HNP) and 0.54 (GSP). Agreement did not improve even when restricting analysis to a single site (true cord, 23 cases), with KAs for 3-tier/2-tier being 0.54/0.48 (all pathologists), $0.52 / 0.47$ (HNP) and 0.58/0.54 (GSP). All $7 \mathrm{HNP}$ agreed on a grade in only 9 cases, while 6/7 HNP agreed on a grade in 15 cases. 6/7 GSP gave the same grade as HNP in only 11 of these $15(73 \%)$ cases.

Conclusions: Overall agreement in grading dysplasia is only moderate at best. Oral pathologists tend to assign higher dysplasia grades, while community pathologists tend to assign lower grades. HNP divide moderate dysplasias equally between high grade and low grade. A better defined and more objective classification system is likely required to improve reproducibility among all pathologists.

\section{Expression of Fas and FasL in Ameloblastoma and Its Different} Subtypes

F Mashhadi Abbas, S Mokhtari, H Ghorbani. Shahid Beheshti University of Medical Sciences, Tehran, Islamic Republic of Iran.

Background: Ameloblastoma originates from dental follicle or other sources of odontogenic epithelium. It is the most important odontogenic neoplasm due to its local aggressive behavior. Fas and FasL are critical factors in apoptosis and their altered expression has been detected in many neoplasms. To clarify the possible role of these factors in progression and invasive behavior of ameloblastoma, we examined the expression of Fas and FasL in dental follicle and different types of ameloblastoma. Design: We analyzed immunohistochemical expression of Fas and FasL antibodies in 11 dental follicles and 56 specimens of ameloblastoma ( 35 conventional-type, 15 unicystic-type and 6 ameloblastic carcinoma). Intensity of stain (Intensity Score) and proportion of stained cells (Proportional Score) for each tumor were evaluated and the sum was described as Total Score. Data were analyzed by Non-parametric KruskalWallis and Mann-Whitney tests.

Results: Fas expression was not seen in dental follicles. $44.6 \%$ and $100 \%$ of ameloblastomas expressed Fas and FasL markers, respectively. Expression of Fas and FasL was significantly higher in ameloblastoma than dental follicle (P value: $F a s=0.01$, FasL $=0.0001$ ). The proportional score of FasL expression was significantly higher in conventional ameloblastoma than other types $(\mathrm{p}=0.003)$. Except this observation, no other important difference of Fas or FasL expression was seen between different types of ameloblastoma.

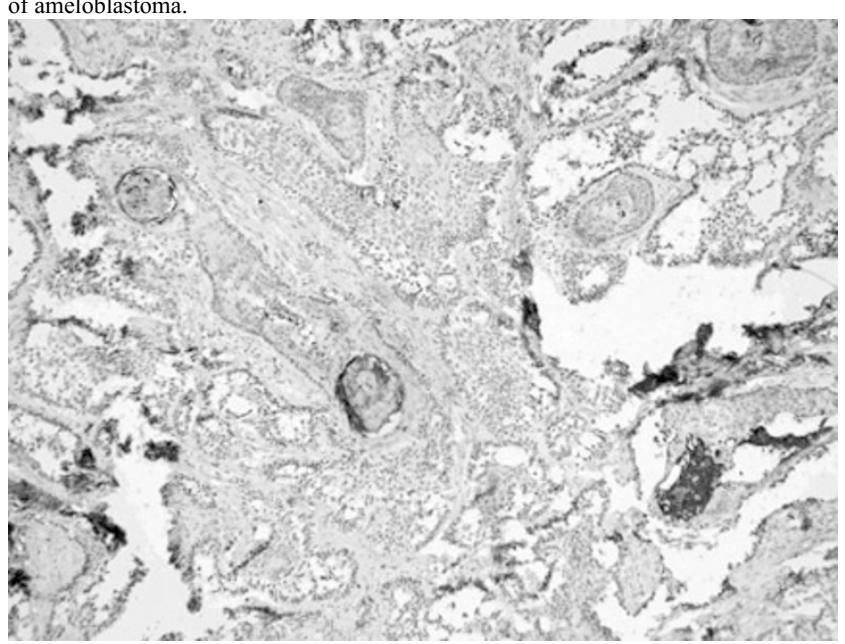




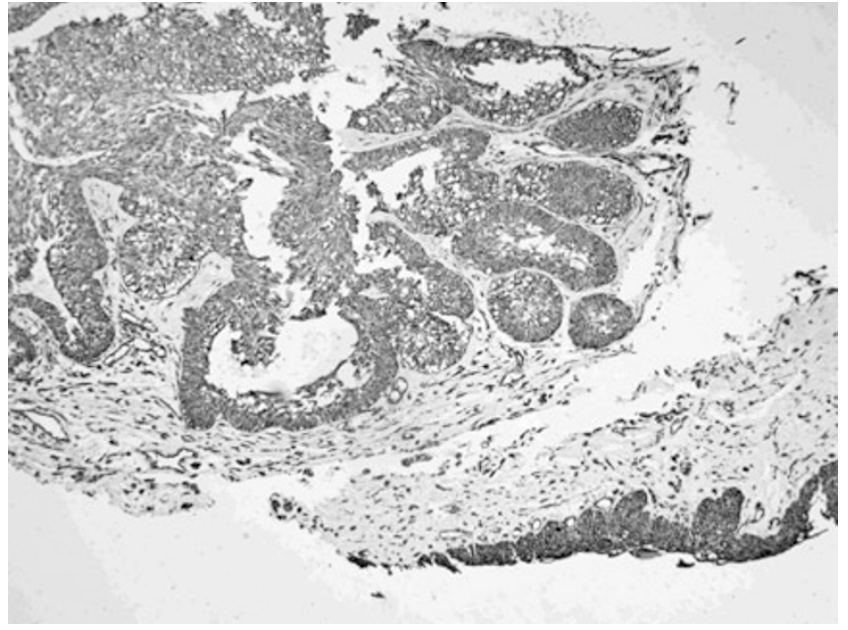

Conclusions: Increased expression of Fas/FasL in ameloblastoma implies that they have critical role in progression and invasion of this tumor. Coexpression of these factors may be due to the presence of an autocrine mechanism of Fas/FasL pathway in ameloblastoma. It seems that Fas/FasL expression is not significantly different in various types of Ameloblastoma. FasL was more expressed than Fas in ameloblastoma specimens; so, it may be more related to its progression and invasive behavior.

1315 Characterization of the Head and Neck Papillary Squamous Cell Carcinoma Variants: Clinicopathological and Molecular Study with Special Reference to Human Papillomavirus (HPV) Association

M Mehrad, DH Carpenter, RD Chernock, H Wang, X-JMa, Y Luo, J Luo, JS Lewis, SK El-Mofty. Washington University School of Medicine, St Louis, MO; Advanced Cell Diagnostics, Inc., Hayward, CA.

Background: Papillary squamous cell carcinoma (PSCC) of the head and neck (H\&N) is often confused with other exophytic mucosal malignancies such as verrucous carcinoma and squamous cell carcinoma with verrucous features. The latter two entities are characterized by excessive keratinization and are broad based. A relationship to HPV infection has been suggested, however, it has not been clearly shown whether PSCC is a homogeneous entity or a spectrum of variants with different clinicopathological features and biological behaviors.

Design: 48 cases of PSCC were retrieved from our surgical pathology database and were reviewed by 3 study pathologists, following morphologic criteria as defined by th WHO. An RNA insitu hybridization (ISH) assay to detect high-risk HPV E6/E7 mRNA was used as were DNA ISH for high-risk HPV and immunohistochemistry (IHC) for p16 and p53. Clinical follow-up data were obtained by chart review.

Results: 7 cases were in the oral cavity (OC), 19 in the oropharynx (OP) and 22 in the larynx. Two morphologic types of PSCC were identified: a keratinizing type (K) in which the epithelial cells showed a maturation trend with minimal surface parakeratin, and a nonkeratinizing type (NK) in which the papillae were completely covered by immature basaloid cells. Transcriptionally active HPV was documented in a number of tumors; The majority of these were found in younger patients and occured more commonly in the OP. They showed NK morphology, were p16 positive and $\mathrm{p} 53$ negative.

\begin{tabular}{|c|c|c|c|c|}
\hline Site $(\mathrm{n})$ & NK \% & p16+\% & p53+\% & HPV + \% \\
\hline OP (19) & 68.4 & 68.4 & 21.1 & 78.9 \\
\hline Oral (7) & 14.3 & $14.3(\mathrm{p}=0.012)$ & $71.4(\mathrm{p}=0.028)$ & $57.1(\mathrm{p}=0.067)$ \\
\hline Larynx (22) & 9.0 & $13.6(\mathrm{p}=0.0005)$ & $68.2(\mathrm{p}=0.004)$ & $42.1(\mathrm{p}=0.020)$ \\
\hline
\end{tabular}

Overall survival and disease-free survival (DFS) did not significantly differ by tumo site, however, HPV-related tumors showed a better DFS trend.

Conclusions: PSCC of the $\mathrm{H} \& \mathrm{~N}$ is a heterogeneous entity with different morphologic, clinicopathologic and molecular variants. A group of PSCC is HPV positive, occurred more commonly in the OP, had NK morphology, was p16 positive and p53 negative. Patient's outcome did not vary significantly by site, however, HPV-related tumors showed a trend towards better DFS.

1316 HPV-Positive Oral Squamous Cell Carcinoma Is More Common in the Oropharynx of Young Male Non-Smokers

T Morgan, J Hebert, A Schilling, N Gross, J Schindler, C Corless, J Hooper. OHSU, Portland; OSHU, Portland.

Background: Recent data suggest that the prevalence of HPV-positive oropharyngeal cancers is increasing. If current trends continue, the annual number of HPV-positive ora cancers is expected to surpass the incidence of cervical cancer by 2020 . The literature is mixed regarding the impact of tumor site and gender on the frequency of HPV-positive cancer. Methods of detection also vary; many pathologists use p16 immunostaining as a surrogate marker for HPV driven oral cancer, which may not be as predictive in the mouth as in the cervix. We compared the frequency of HPV-positive oral cancer at various biopsy sites and between patient genders. We also tested the predictive power of p16 compared with PCR and commonly employed high risk HPV tests.

Design: Retrospective analysis of 118 oral cancer biopsies collected at OHSU from 2004-2010 was performed. Biopsy site, patient age, gender, and smoking history were available for 87 cases. All H\&E biopsies were reviewed to confirm diagnoses and all p16 ( $\mathrm{mtm} /$ Roche $)$ immunostained sections were scored as either positive or negative by two independent surgical pathologists. DNA was extracted from tumor blocks to obtain complete HPV genotype by PCR analysis (Diatherix), and high risk HPV screening by hybrid capture (Qiagen hc2) and Cervista (Hologic). A HPV result was considered positive if at least two HPV tests agreed. Results were analyzed by Chi-square analysis and ANOVA with post hoc testing.

Results: HPV-positive oral cancers were most common in the tonsil and base of tongue (oropharynx) biopsies $(\mathrm{p}<0.0001)$ of younger male $(55$ years vs 65 years) $(\mathrm{p}=0.0002)$ non-smokers $(\mathrm{p}=0.006)$. HPV was less common in oral cancer in females $(6 / 23,26 \%)$ compared with males $(30 / 64,47 \%)$. P16 staining had excellent negative predictive value (94 [83-98]) and good positive predictive value (87 [71-95]) compared to HPV DNA results. Most positive cases were HPV16 (27/33). PCR yielded two false negatives compared to hc2 and Cervista, perhaps due to paraffin extraction.

\begin{tabular}{|l|l|l|l||}
\hline \multicolumn{1}{|l|}{} & HPV+ by PCR & HPV+by hc2 & P16 positive \\
\hline Oropharynx (n=44) & $72 \%$ & $75 \%$ & $80 \%$ \\
\hline Larynx (n=19) & $0 \%$ & $5 \%$ & $0 \%$ \\
\hline Floor of Mouth (n=10) & $0 \%$ & $0 \%$ & $10 \%$ \\
\hline Roof of Mouth (n=6) & $17 \%$ & $17 \%$ & $0 \%$ \\
\hline Tongue (n=8) & $13 \%$ & $13 \%$ \\
\hline
\end{tabular}

Conclusions: Our data suggest HPV-positive cancers are more common in the oropharynx of young male non-smokers. High risk HPV assays commonly employed for cervical screening such as hc2 and Cervista detected more cases than PCR with fewer false positives than 16 immunostaining.

1317 Sox10 Is a Novel Marker of Acinus and Intercalated Duct Differentiation in Salivary Gland Tumors

R Ohtomo, T Mori, K Tsuta, AM Maeshima, S Yoshimoto, M Asai, H Tsuda. National Cancer Center Hospital, Tokyo, Japan.

Background: Sox10 is known as a transcription factor crucial for specification and maturation of the neural crest, and maintenance of Schwann cells and melanocytes. In a murine model, Sox10 is also shown to be highly expressed in myoepithelial and acinic cells at the salivary glands. However, there are few reports that studied Sox 10 expression in human salivary glands and tumors.

Design: We collected formalin-fixed paraffin-embedded specimens of 15 unremarkable salivary gland tissues (12 parotid glands and 3 submandibular glands) and 15 tumor tissues resected surgically between 1998 and 2010. These tumors comprised of 5 acinic cell carcinomas (AciCC), 3 adenoid cystic carcinoma (ACC), and one case each of epithelialmyoepithelial carcinoma (EMC), salivary duct carcinoma (SDC), mucoepidermoid carcinoma (MEC), carcinoma ex pleomorphic adenoma (Ca-Ex-PA), carcinoma not otherwise specified (CaNOS), pleomorphic adenoma (PA), myoepithelioma (ME) The expression of Sox 10, p63 and SMA were immunohistochemically examined and correlated with all types of these salivary glands and tumors.

Results: In all 15 unremarkable salivary glands, Sox10 was expressed in the nuclei of most $(>80 \%)$ of the myoepithelial cells and luminal (epithelial) cells in acini and intercalated ducts, but not expressed in basal cells and luminal cells of striated and excretory ducts. p63 was confirmed to be expressed in myoepithelial and basal cells but not in all luminal cells, and SMA was confirmed to be expressed only in myoepithelial cells. And Sox 10 was expressed luminal and myoepithelial cells of all cases of AciCC ACC, EMC, and PA, but was completely negative in the cases of SDC, MEC, and CaNOS. In a ME, Sox10 was expressed in myoepithelial cells.

Conclusions: Sox 10 was expressed in most of the luminal and abluminal cells of acin and intercalated ducts in all human salivary glands examined. In the salivary tumors, Sox 10 was positive in tumors that have similarity to acinus or intercalated duct, i.e. AciCCs, ACCs, EMC, and ME, but negative in those that have similarity to striated and excretory duct, i.e., SDC and MEC. Sox10 can be one of useful markers for the identification of cellular genealogy and diagnosis of salivary gland tumors.

1318 HPV-31 Is the Most Common HPV Subtype Isolated from Oropharyngeal Squamous Cell Carcinomas in South Africa

C Paquette, MF Evans, S Meer, V Rajendran, CS Adamson, K Cooper. Fletcher Allen Health Care, Burlington, VT; University of Vermont, Burlington, VT; University of the Witwatersrand, Johannesburg, South Africa.

Background: Oropharyngeal squamous cell carcinomas (OSCCs) are associated with two main risk pathways: chemical (tobacco and alcohol) and human papillomavirus (HPV) infection. For the latter, HPV-16 has been implicated as the key etiologic subtype worldwide. However, data are scarce regarding OSCC in South African (SA) patients; three prior studies suggested no significant role for the virus, in contrast to worldwide trends. We aimed to assess SA OSCCs for HPV, determine virus genotype, and investigate $\mathrm{p} 16^{\mathrm{INK} 4 \mathrm{a}}$ immunohistochemical (IHC) positivity as a marker for HPV driven tumors.

Design: Formalin-fixed, paraffin-embedded tissues from 67 OSCCs collected between 2005 and 2010 from South African patients were analyzed for HPV DNA via PCR (positive results genotyped by sequencing), chromogenic in situ hybridization (CISH), and $\mathrm{p} 16^{\mathrm{INK} 4 \mathrm{a}} \mathrm{IHC}$. Two cases were excluded (non-diagnostic tissue and respiratory papillomatosis).

Results: We analyzed 65 OSCCs from 54 patients (mean age $58,80 \%$ male, $76 \%$ black and $24 \%$ Caucasian). Of the samples $48(74 \%)$ were HPV positive by PCR: 34 (71\%) HPV-31, 6 (12\%) HPV-16, 4 (8\%) HPV-18, 2 (4\%) 'untypeable' and one each (4\%) combined HPV-16/31 or HPV-18/31. CISH was negative in all cases. Of the $34 \mathrm{p} 16^{\text {INK4 }}$ IHC positive cases, $26(76 \%)$ were positive for HPV by PCR. Of the $31 \mathrm{p} 16^{\mathrm{INK} 4 \mathrm{a}} \mathrm{IHC}$ negative cases, 22 (71\%) cases were positive for HPV by PCR.

Conclusions: In accordance with worldwide epidemiologic data, HPV appears to play a role in the pathogenesis of OSCC in South Africa, with strongest evidence for OSCCs positive for both $16^{\text {INK4a }} \mathrm{IHC}$ and HPV by PCR, comprising $40 \%$ of our tumor samples. The $\mathrm{p} 16^{\mathrm{INK} 4 \mathrm{a}} \mathrm{IHC}$ stain is used as a marker for HPV-related tumors, and may 
help distinguish HPV-driven tumors from tumors with incidental HPV. Contrary to the results of meta-analyses derived primarily from Western patient cohorts, HPV-31, and not HPV-16, was the predominant genotype identified. The absence of specific assays for HPV-31 in previous SA studies may explain our present findings. Further studies, including HPV-31 viral load and alternative CISH methodologies, must be investigated to corroborate our data.

1319 Verrucous Carcinoma of the Head and Neck Lacks TranscriptionallyActive Human Papillomavirus

KR Patel, RD Chernock, T Zhang, X Wang, SK El-Mofty, JS Lewis. Washington University in St. Louis, St. Louis, MO.

Background: Studies have shown the presence of transcriptionally-active human papillomavirus (HPV) in squamous cell carcinomas (SCC) and in numerous SCC variants when they occur in the oropharynx. While HPV DNA can be found in many nonoropharyngeal SCC, transcriptionally-active HPV is quite rare. Verrucous carcinomas (VC) are a subtype of SCC characterized by warty appearance, locally destructive growth pattern, and lack of metastasis when not associated with an invasive SCC component. A few studies have shown variable amounts of HPV DNA and also p16 protein expression in verrucous lesions of the head and neck. However, no definitive link has been established between these tumors and HPV. In particular, VC has not been assessed for transcriptionally active HPV (mRNA).

Design: We performed a search of our pathology database for the keyword "verrucous" and after reviewing all cases, selected 49 which met our diagnostic criteria. The lesions were categorized into 3 groups on the basis of histologic features: pure VC (26/49), well differentiated SCC with verrucous features (SCC-VF) (17/49) and SCC arising in VC (SCC-VC) (6/49). SCC-VF were verrucous carcinomas with dysplasia or with minor invasive SCC $(<2 \mathrm{~mm}$ in depth). SCC-VC was $\mathrm{VC}$ with major invasive $\mathrm{SCC}(>2 \mathrm{~mm}$ in depth). p16 immunostaining and RT-PCR for E6 and E7 mRNA of 13 high risk HPV types were performed. p16 was interpreted as positive only with both cytoplasmic and nuclear staining and was graded in quartiles for extent.

Results: Table 1 shows the p16 immunohistochemistry results by type.

Table 1 - p16 immunoreactivity by tumor type.

\begin{tabular}{|c|c|c|c|c|c|}
\hline p16 Staining Extent & 0 & $1+(1-25 \%)$ & $2+(26-50 \%)$ & $3+(51-75 \%)$ & $4+(76-100 \%)$ \\
\hline VE & $10(39 \%)$ & $8(31 \%)$ & $5(19 \%)$ & $1(4 \%)$ & $2(8 \%)$ \\
\hline SCC-VF & $7(41 \%)$ & $6(35 \%)$ & $2(12 \%)$ & 0 & $2(12 \%)$ \\
\hline $8 C-\mathrm{VC}$ & $4(67 \%)$ & $1(17 \%)$ & & $1(17 \%)$ & \\
\hline
\end{tabular}

All 23 cases which could be tested (including 15 VC, 6 SCC-VF, and 2 SCC-VC) were negative for HPV mRNA by RT-PCR.

Conclusions: $\mathrm{p} 16$ is expressed in more than $50 \%$ of $\mathrm{VC}$ and related lesions, although it is not usually extensive. However, by sensitive detection methods, transcriptionallyactive HPV is absent. These findings suggest that HPV does not have biological or clinical significance in $\mathrm{VC}$ or its related lesions.

1320 Cancer/Testis (CT) Antigens and p53 as Potential Diagnostic Markers in Squamous Cell Carcinomas of the Head and Neck

KC Piotti, T Scognamiglio, $R$ Chiu, Y-T Chen. Weill Cornell Medical College, New York, NY; Ludwig Institute for Cancer Research, New York, NY

Background: The diagnosis of squamous dysplasia in head and neck $(H \& N)$ biopsies is subjective and no markers exist to reliably identify a clonal squamous proliferation. $\mathrm{CT}$ antigens comprise a group of proteins that are normally only expressed in germ cells but can be aberrantly activated in cancers, making them potential diagnostic markers. Similarly, p53 gene mutation and protein overexpression is a crucial and clonal event in carcinogenesis. In this study, we evaluated the frequency of CT and $\mathrm{p} 53$ expression in $\mathrm{H} \& \mathrm{~N}$ squamous cell carcinoma (SqCC) and sought their potential as diagnostic markers in biopsy specimens.

Design: Protein expression of $8 \mathrm{CT}$ antigens (MAGEA, GAGE, SAGE1, NY-ESO-1, CT7, CT10, CT45 and NXF2) and p53 was immunohistochemically evaluated in a tissue microarray of $76 \mathrm{H} \& \mathrm{~N}$ SqCC. 118 biopsy specimens with variable degrees of squamous dysplasia, obtained from 87 cases, were examined on whole sections for 6 CT antigens (MAGEA, GAGE, NY-ESO-1, CT7, CT10, and CT45, in an antibody cocktail) and p53. Unequivocal CT antigen staining in any tumor cell and nuclear p53 staining in $>20 \%$ of tumor cells were interpreted as positive.

Results: Fifty of 76 invasive SqCC (66\%) expressed at least one CT antigen. Overexpression of p53 was found in 27 of 76 cases $(36 \%)$. Sixty-two SqCC $(82 \%)$ expressed CT antigen and/or p53. Among the 118 biopsy specimens, 9 were CT positive, 4 were p53 positive, and none was double positive. Of the papillary lesions, 1 (with moderate dysplasia) of 55 was focally CT positive and none were $\mathrm{p} 53$ positive. In contrast, of the 65 flat lesions, 8 were CT positive and 4 were p53 positive. These 12 specimens ranged from mild dysplasia to carcinoma in situ histologically and were from 10 patients, 6 of which had a prior or concurrent diagnosis of SqCC. (Figure: moderate dysplasia in a patient with prior $\mathrm{SqCC}$, positive for $\mathrm{CT}$ expression).

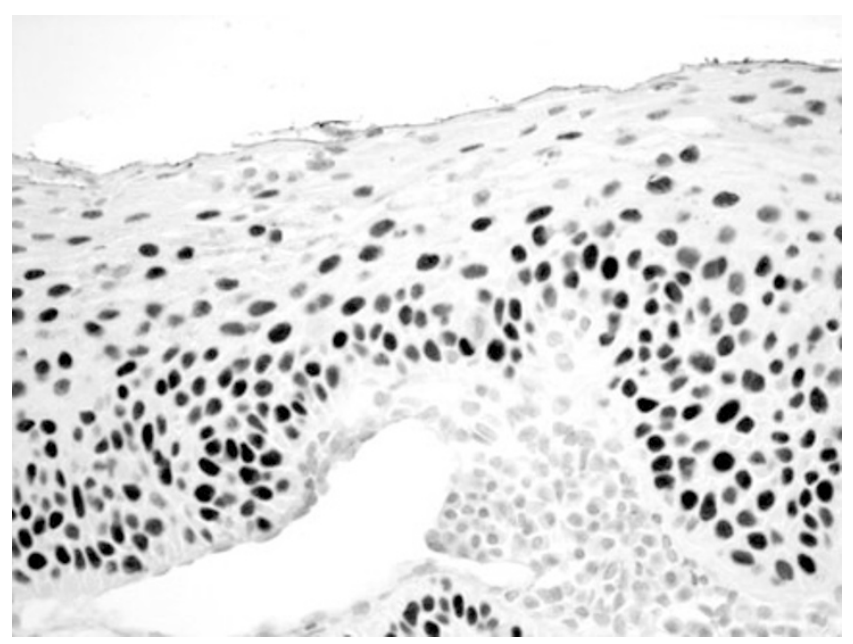

Conclusions: More than $80 \%$ of invasive $\mathrm{H} \& \mathrm{~N} \mathrm{SqCC}$ showed CT antigen expression and/or p53 overexpression. Although lack of CT/p53 expression is not diagnostically useful, positive $\mathrm{CT}$ or $\mathrm{p} 53$ immunostains may serve as a specific marker for malignant transformation, distinguishing these lesions from squamous lesions with benign atypia.

1321 Squamous Morular Metaplasia in Non-Intestinal Type Sinonasal Adenocarcinoma and Seromucinous Hamartomas: A Novel Immunophenotypic Pitfall

BM Purgina, JM Bastaki, RR Seethala. University of Pittsburgh Medical Center, Pittsburgh, PA.

Background: Non-intestinal type sinonasal adenocarcinomas (non-ITAC) and intestinal type adenocarcinomas (ITAC) are rare biologically distinct sinonasal tumors that can be immunophenotypically distinguished using markers like CK7, CK20, and CDX2. We had surprisingly noted one case of sinonasal seromucinous hamartoma (SSH), a potential precursor to non-ITAC, which showed focal CDX2 immunoreactivity in association with squamoid morules. To survey this potential diagnostic pitfall, we evaluated the morphologic and immunohistochemical characteristics of a series of SSH, non-ITAC, and ITAC.

Design: Three SSH, fifteen (9 low grade (LG), 6 high grade (HG)) non-ITAC (5 papillary, 3 lobular, 2 cystic, 4 solid and 1 cribriform), and sixteen ITAC (4 papillary, 5 colonic, 1 solid, 6 mucinous) were retrieved from our files. One case of SSH arising in left middle turbinate was associated with a non-ITAC of the right sinonasal cavity. Histologic features, including growth pattern, metaplastic changes, and immunohistochemical profile were tabulated.

Results: All SSH (3/3) were CK7 positive, S100 positive, and CK20 negative. One $\mathrm{SSH}$ with morular metaplasia showed CDX2 positivity restricted to morules. All ITACs tested were CK20 positive (10/10), CDX2 positive (6/6), and 9/10 were CK7 positive. Squamous or morular metaplasias were not seen in ITAC. All non-ITACs tested were CK7 positive and CK20 negative (9/9). A subset of LG non-ITACs (3/5) were S100 positive, similar to SSH, and DOG1 (an acinar selective marker) positive. Three cases showed squamoid features accompanied by focal p 63 and CK 5/6 reactivity. One nonITAC with metaplasia showed morules with CDX2 and nuclear beta-catenin positivity. The other cases with squamous metaplasia were CDX2 negative.

Conclusions: SSH and non-ITAC add to the list of glandular lesions that may show morular metaplasia. Similar to other tumors, the morules are characterized by nuclear beta catenin and CDX2 reactivity which is a potential diagnostic pitfall in the sinonasal tract. However, the restriction to morules and lack of CK20 reactivity distinguish SSH/non-ITAC with morular metaplasia from ITAC. The shared morphology and immunophenotype between SSH and a subset of non-ITAC supports an etiologic relationship between these entities.

1322 Validation of Transcriptionally Active HPV in Salivary Mucoepidermoid Carcinomas

$Z$ Ren, $R$ Li, T Isayeva, S Bai, $N$ Said-Al-Naief, M Brandwein-Gensler. University of Alabama at Birmingham, Birmingham, AL; University of the Pacific, San Francisco, CA. Background: Mucoepidermoid carcinoma (MEC) is a common salivary malignancy. We have recently demonstrated expression of HPV16 and HPV18 specific E6/E7 transcripts in $30.2 \%$, and $20.5 \%$ of MEC, respectively, by nested RT-PCR. Here we validate these findings by demonstrating HR HPV E6 protein in MEC by immunofluorescence (IF). In addition, we compared p16 expression with HPV status in these tumors.

Design: We studied 84 MEC with known HPV status. E6 protein was determined by mouse monoclonal HPV16/18 E6 (C1P5) primary antibody and goat anti-mouse secondary antibody conjugated to Alexa 488 . Immunohistochemistry stains were performed for $\mathrm{p} 16$, and expression was categorized for staining intensity (scale $0-4$ ) and percent staining distribution, for the nuclei and cytoplasm of the squamous and mucinous tumor elements. All slides were examined blinded to HPV status by two pathologists. Results: E6 protein was demonstrated in 18/84 MEC (21\%), with either nuclear and/ or cytoplasmic staining in both mucinous and squamoid tumor elements, as shown in figure 1 (arrows). 


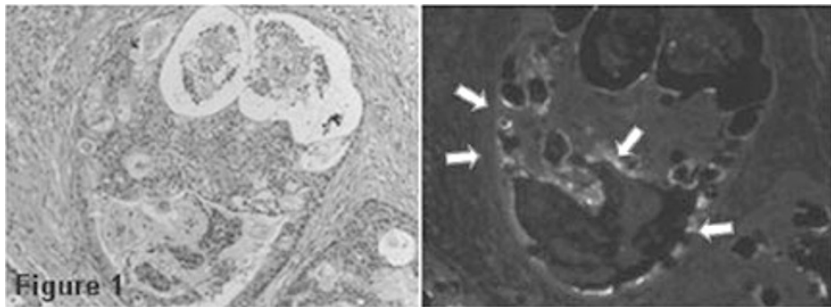

All E6+ MEC were HPV16/18+ by RT-PCR. Fourteen additional tumors were negative by IF and positive by RT-PCR (sensitivity $55 \%$, specificity $100 \%$ ). For p16 staining, $15 / 84$ MEC $(18 \%)$ demonstrated strong $(+4)$ nuclear and cytoplasmic p16 expression in $\geq 50 \%$ of solid MEC components; $53 / 84$ MEC (64\%) demonstrated strong ( +4$)$ nuclear and cytoplasmic p16 expression in $\geq 50 \%$ of the mucinous glandular components. No correlation, however, was seen between p16 expression and HPV status.

Conclusions: Here we validate our previous RT-PCR data demonstrating transcriptionally active HPV16/18 in MEC, by detecting E6 viral oncoprotein in tumor cells. As expected, the sensitivity of IF is lower than RT-PCR, but the specificity is high These findings support the concept that HPV 16/18 promotes the carcinogenesis of MEC. Interestingly, we found no relationship between transcriptionally active HPV status and p16 $6^{\mathrm{INK} 4 \mathrm{a}}$ expression in MEC, in contrast to the paradigm of HPV-mediated tonsillar cancers. Lack of p16 overexpression in transcriptionally active HPV16/18 MEC may be attributed promoter hypermethylation, or deletions and point mutations in $\mathrm{p} 16^{\mathrm{INK} 4 \mathrm{a}}$

1323 IgG4-Related Sialadenitis Is Rare: Immunohistological Investigation of 105 Cases of Chronic Submandibular Sialadenitis

M Rodriguez-Justo, JD Harrison. University College London Hospitals \& Faculty of Biomedical Sciences UCL, London, United Kingdom; King's College London Dental Institute, London, United Kingdom.

Background: Chronic sialadenitis is a relatively common disease in which microliths play an important role in its pathogenesis. The suggestion that all cases of chronic sclerosing sialadenitis are IgG4-related sialadenitis is not supported by evidence, and we have recently reported a case with the histological features of chronic sclerosing sialadenitis that lacked significant numbers of IgG4 plasma cells (Histopathology 2011, 58: 1164-6)

Design: We have undertaken an investigation of an archive of 105 cases of chronic submandibular sialadenitis that had been previously extensively characterized (Histopathology 1997, 31:237-51). The degree of fibrosis, periductal lymphoplasmacytic infiltrate, and the presence or absence of liths, granulocytic epithelial lesions (GEL) and phlebitis were assessed. The number of IgG4 plasma cells was counted in $10 \mathrm{HPFs}$ and samples were classified as " 0 ": no IgG4 plasma cells, "1": scattered IgG4 cells and; " 2 ": > 50 IgG4 cells per HPF. The ratio IgG4:IgG was also investigated.

Results: The degrees of inflammation, atrophy and fibrosis varied greatly, and 67 cases were classified as chronic sclerosing sialadenitis. Sialoliths were present in 63 out of 89 cases in which the presence or absence of sialolithiasis could be confirmed. The number of IgG4 plasma cells was " 0 " in 89 cases, " 1 " in 19 cases, and " 2 " in three cases, in which there was sialolithiasis. There were relatively more widespread atrophy, fibrosis and inflammation and more sialoliths in glands with IgG4 cells described as 1 than in glands with 0 . There was widespread atrophy, fibrosis and inflammation in the 3 glands with IgG4 cells above 50 per HPF, which surrounded the ducts that contained sialoliths. Conclusions: The incidence of IgG4-related sialadenitis is unknown with about 52 cases reported in the literature. However, our investigation adds further evidence that IgG4-related sialadenitis is rare. In addition, our findings raise the possibility that IgG4 plasma cells represent a local reaction to sialolithiasis rather than a systemic process in the three cases with significant numbers of IgG4 plasma cells.

1324 ERCC1, P16 and Ki-67 Immunohistochemichal Expression as Predictive and Prognostic Marker in Head and Neck Squamous Cell Carcinoma Treated with Platin-Based Induction Chemotherapy. Staining with the Two Anti-ERCC1 Antibodies (8F1 and FL-297) Was Compared H Roussel, P Ravel, H Tournat, $M$ Housset, S Hans, P Bruneval, C Badoual. Hôpita Européen Georges Pompidou, Université Paris-Descartes, Paris, France; Centre National de la Recherche Scientifique (CNRS), Unité Mixte de Recherche (UMR 554) et Université, Montpellier, France.

Background: Head and neck squamous cell carcinoma (HNSCC) treatment had undergone important changes, in particular due to the organ preservation and the advent of chemotherapy. The excision repair cross-complementation group 1 (ERCC1) enzyme plays a rate-limiting role in the nucleotide excision repair pathway and has been associated with resistance to platinum-based chemotherapy. The purpose of this study was to evaluate the role of ERCC1 expression with $\mathrm{p} 16$ and $\mathrm{Ki}-67$ as predictive marker in the response to platinum-based induction chemotherapy in patients with HNSCC. Design: 208 patients treated from 2000 to 2006 by an induction chemotherapy regimen for HNSCC were included retrospectively. ERCC1 (8F1 and FL297 clone), p16 and $\mathrm{Ki}-67$ staining were performed on paraffin-embedded tumor tissue collected before chemotherapy. We assessed response to treatment, progression-free survival (PFS) and overall survival (OS).

Results: Respectively, 68\% (142/208 patients) and 81.5\% (163/200 patients) of HNSCC showed low expression of 8F1 and FL297 ERCC1. No correlation was found between the two clones $(\mathrm{p}=0.1)$. There was no correlation between the expression of 8F1 ERCC1, FL297 ERCC1 and Ki-67 and the response to induction chemotherapy, OS or PFS. However, in the subgroup of 129 patients treated with induction cisplatin$5 \mathrm{FU}$ chemotherapy, a low expression of $8 \mathrm{~F} 1 \mathrm{ERCC} 1$ was significantly associated with a better response $(\mathrm{p}=0.027)$. Over expression of $\mathrm{p} 16$, found in $34.5 \%$ of cancers of the oropharynx was significantly correlated with a better OS $(\mathrm{p}=0.0007)$ and a better PFS $(\mathrm{p}=0.01)$

Conclusions: These results suggest that ERCC1 expression might be a useful predictive marker of HNSCC in patients treated by cisplatin-based chemotherapy. The 8F1 ERCC1 clone appears to be the best for immunohistochemical analysis; clone FL297 did not show any association with treatment response and survival. Independently, our study confirms the prognostic value of the over expression of p16 in carcinoma of the oropharynx.

1325 Cyclin D1 Expression as a Prognostic Marker in Oropharyngeal Squamous Cell Carcinoma

JB Scantlebury, JLuo, WL Thorstad, SK El-Mofty, JS Lewis, Jr. Washington University Saint Louis, Saint Louis, MO.

Background: Human papillomavirus (HPV)-related oropharyngeal squamous cell carcinoma (OSCC) is a well-established entity with unique biology and improved prognosis. A new focus is to identify prognostic biomarkers specifically in this HPV positive cohort for use in determining appropriate therapy. Cyclin D1 expression has been suggested as being useful for this purpose.

Design: Immunohistochemistry was performed for cyclin D1 on a tissue microarray cohort of OSCC cases with known clinical follow up and p16 and HPV status (by RNA in situ hybridization/ISH). p16 positivity was defined as positive if $\geq 50 \%$ staining. Cyclin D1 staining was assessed visually for $\%$ positive cells by two study pathologist (JBS and JSL) and also by digital image analysis using Aperio's membrane algorithm, version 9. Cyclin D1 positivity was defined as $\geq 30 \%$ positive tumor cell staining. Results: By visual analysis, $170(84.2 \%)$ cases were cyclin D1 negative and $32(15.8 \%)$ cases were positive. By image analysis, $167(82.7 \%)$ cases were negative and 33 $(16.3 \%)$ positive. The two methods of analysis were strongly correlated (Spearman's rank correlation coefficient $=0.77)$. Of these cases, $150(79.4 \%)$ were HPV RNA ISH positive and $39(20.6 \%)$ negative. Negative cyclin D1 (by either method) correlated significantly $(\mathrm{p}<0.05)$ with $\mathrm{p} 16$ and HPV positivity, with lower T-stage and higher $\mathrm{N}$-stage, and also with non-smoking status. Positive cyclin D1 (be either method) correlated significantly with local and regional failure $(\mathrm{p}<0.05$ for each) but not with distant metastases (visual $\mathrm{p}=0.33$; digital $\mathrm{p}=0.12$ ). In univariate analysis, negative cyclin D1 correlated strongly with better overall survival (visual $\mathrm{p}=1.56 \times 10^{-5}$; digital $\mathrm{p}=4.58 \times 10^{-7}$ ), but in multivariate it did not by either method ( $\mathrm{p}>0.1$ for each). In just the HPV RNA ISH positive patients, negative cyclin D1 also did not correlate significantly with overall survival (visual $\mathrm{p}=0.58$; digital $\mathrm{p}=0.067$ )

Conclusions: Cyclin D1 expression (as $<$ versus $>30 \%$ ) correlates strongly with patient outcomes in OSCC. However, this correlation appears to be due to high correlation with HPV status and other prognostic variables so that cyclin D1 does not predict prognosis among just the HPV/p16 positive population.

\section{Salivary Gland Myoepitheliomas Are Not Associated with EWSR 1} Genetic Rearrangements

AA Shah, MJ Mentrikoski, HY Scruggs, RD Legallo, HF Frierson, SE Mills, EB Stelow. University of Virginia, Charlottesville.

Background: The Ewing sarcoma breakpoint region 1 (EWSR1) is a well-known gene involved in sarcoma translocations. Recently EWSR1 gene rearrangements have been reported as common events in "myoepithelial tumors" arising outside the salivary glands. Subsequently, EWSR1 gene rearrangements were also described in hyalinizing clear-cell carcinomas of the salivary glands and upper aerodigestive tract. This study examines the presence of EWSR 1 rearrangement in a variety of salivary gland lesions many of which have a myoepithelial phenotype.

Design: A tissue microarray containing normal salivary gland, 3 polymorphous lowgrade adenocarcinomas, 4 acinic cell carcinomas, 4 salivary duct carcinomas, 16 pleomorphic adenomas, 9 Warthin tumors, 10 mucoepidermoid carcinomas and 21 adenoid cystic carcinomas was assessed by Fluorescent in-situ hybridization (FISH) for the EWSR1 rearrangement. Additionally, 5 parotid gland myoepitheliomas were also assessed by FISH. Following pretreatment and digestion the deparaffinized tissue sections were hybridized with the Vysis LSI EWSR1 Dual Color Breakapart DNA probes. Tissue from an Ewing sarcoma was used as a positive control. Interphase nuclei were scored using a fluorescent scope and 100x oil immersion lens. 100 cells were scored as "intact" or "disrupted". A tumor is considered rearranged for the specific gene if greater than $10 \%$ of tumor cells are disrupted.

Results: The EWSR1 gene was intact in all benign and malignant salivary gland lesions including the myoepitheliomas. The EWSR1 rearrangement was detected in the positive control Ewing sarcoma.

Conclusions: The EWSR1 rearrangement is not a feature of the vast majority of salivary gland lesions including myoepitheliomas, pleomorphic adenomas, acinic cell carcinomas, mucoepidermoid carcinomas, adenoid cystic carcinomas, Warthin tumors, salivary duct carcinomas or polymorphous low-grade adenocarcinomas. These findings suggest that salivary gland myoepithelial tumors and their soft tissue counterparts are distinct entities that do not share a pathogenetic relationship.

1327 Lobular Capillary Hemangiomas of the Sinonasal Tract: Findings in a Contemporary Series with Emphasis on Recurrence

SC Smith, RM Patel, DR Lucas, JB McHugh. University of Michigan, Ann Arbor, MI. Background: Lobular capillary hemangioma (LCH) was defined by Mills et al. in 1980, as a distinctive lesion and subset of vascular lesions of the oral and mucosal membranes generally diagnosed as "pyogenic granuloma" (PG) (1). These constituted a minority of vascular lesions in the head and neck, but included cases in the sinonasal mucosae, with recurrence after excision noted in as many as $10 \%$. However, a contemporary series 
observed lack of recurrence of sinonasal LCHs (2). As our experience and case reports have observed difficulty in extirpation of these lesions, we performed a clinicopathologic institutional review of sinonasal LCH.

Design: With IRB approval, we performed a systematic search of our institution's case files and medical record system, reviewing and tabulating clinicopathologic, histologic, and follow-up parameters of nasal or sinus lesions diagnosed as LCH or PG between 1989 and 2009. Lesions meeting Mills et al. criteria were included. Statistical analyses included two sample tests, chi-square tests, and Kaplan-Meier Analysis.

Results: Of cases identified, 34 of $45(76 \%)$ met criteria for LCH. Presenting symptoms included epistaxis $(78 \%)$, obstruction $(34 \%)$, and pain $(3 \%)$. No sex predilection was observed $(17 / 18 ; \mathrm{M} / \mathrm{F})$, nor was any difference in distribution of ages by $\operatorname{sex}(\mathrm{p}=0.97)$ or of sex by age group (pediatric, reproductive age, and $>40$ years, $p=0.48$ ), with a median age of 39 years. Pregnancy was associated with 5/34 (15\%) cases, while antecedent trauma was reported in 4/34 (12\%). Histologically, ulceration was identified in 23 of $34(68 \%)$ and prominent mitotic activity ( $>10 / 10 \mathrm{HPF})$ in 3 of $34(9 \%)$. Cellularity of lobules was low in $8 / 34$, moderate in $22 / 34$, and dense in $4 / 34$. In terms of recurrence free survival over a median follow-up of 36 months, we observed recurrence after excision in 13 of 34 cases $(38 \%)$, including cases of clinically treated, unbiopsied recurrence $(7 / 34,21 \%)$ and biopsy-documented recurrence $(6 / 34,18 \%)$.

Conclusions: Review of a contemporary series of LCH observed similar distributions of age and sex, presenting symptomatology, and association with pregnancy to prior studies. In contrast to the only recent series (2), recurrence in this cohort was more frequent, comparable to that originally reported. Our findings suggest sinonasal LCH demonstrates greater local tenacity than previously appreciated. Awareness of this may aid in avoiding misdiagnosis of these lesions as other vascular lesions such as angiofibroma and angiosarcoma.

1. Mills et al. Am J Surg Path 1980.

2. Puxeddu et al. Am J Rhin 2006.

1328 Chromosomal Instability Predicts the Progression of Premalignant Head and Neck Lesions

E-JM Speel, EVE Bergshoeff. TJH Siebers, SAJHM Fleskens, A Haesevoets, JAWM van der Laak, TMAW Merkx, RP Takes, PJ Slootweg, JJ Manni, B Kremer. Maastricht University Medical Center, Maastricht, Netherlands; Atrium Medical Center, Heerlen, Netherlands Antilles; St Radboud University Medical Center, Nijmegen, Netherlands. Background: A major dilemma in the management of patients with precursor lesions of the head and neck mucosa lies in deciding which lesions will progress into carcinoma. Currently used treatments are guided by histopathological examination, which however is troubled by inter-observer variability. The aim of this study was to evaluate the value of chromosomal instability (CIN) detected by fluorescence in situ hybridization (FISH) for the identification of head and neck premalignant lesions at risk for progression.

Design: We examined a series of premalignant oral mucosa lesions ( $\mathrm{n}=106$ patients) and laryngeal precursor lesions ( $\mathrm{n}=75$ patients) by means of FISH on paraffin-embedded tissue sections using chromosome 1 and 7 -specific centromere probes. Cell nuclei were analyzed for CIN in the fluorescence microscope, indicated by the presence of chromosome imbalances and/or polyploidization. Results were correlated with histopathological data as well as with collected clinical follow-up data.

Results: In both oral and laryngeal precursor lesions, the 5-year progression-free survival rate was $\sim 90 \%$. Outcome of routine histopathology did only predict malignant progression when comparing severe dysplasia with lower stage precursor lesions (hyperplasia, mild en moderate dysplasia) $(\mathrm{p} \leq 0.038)$. CIN was detected in all different subgroups of histopathological differentiation. Moreover, the percentage of precursor lesions harboring CIN increased with progressing histopathological stage. CIN was significantly associated with a lower progression-free survival as compared with lesions without CIN ( $\mathrm{p}<0.001)$.

Conclusions: The tumorigenesis of the head and neck mucosa is associated with the development of CIN, which can reliably identify lesions at risk for malignant progression. Therefore, intensive follow-up and/or a more aggressive treatment of lesions with CIN should be considered, and for example examined in prospective studies.

1329 Analysis of HPV Integration Sites in Oropharyngeal Squamous Cell Carcinomas

E-JM Speel, CU Huebbers, NC Olthof, J Kolligs, SF Preuss, U Drebber, B Kremer, JP Klussmann. Maastricht University Medical Center, Maastricht, Netherlands; University of Cologne, Cologne, Germany; University Hospital Giessen, Giessen, Germany.

Background: Human papillomavirus (HPV) is an independent risk factor for the development of up to $50 \%$ of oropharyngeal squamous cell carcinomas (OSCC). They are mainly associated with HPV16. HPV-positive tumors show typical histopathological and molecular features, and patients show less alcohol and tobacco intake and a more favorable survival than the HPV-negative entity of tumors. Controversy exists on the physical status of the virus in OSCC and on HPV integration being an essential factor for tumor development. Whereas in cervical intraepithelial neoplasia lesions oncogenic HPV integrates into the cellular genome upon transition to carcinoma, for HPV-related OSCC this is unclear.

Design: Our research group has collected a series of 66 HPV-positive OSCC and 6 cell lines. The physical status of these samples was analysed with a set of independent techniques to detect viral integration, including APOT- and DIPS-PCR to detect virushuman fusion sites, the new MLPA-PCR technology to quantify viral load and ratios between HPV oncogenes E6/E7 and E2, and FISH

Results: HPV16 integration in the cellular genome was identified in $42 \%$ of OSCC. HPV integration sites were distributed all over the genome and were preferably found in gene loci, often with reported involvement in tumorigenesis, including BCL2, FANCC, TRAF3 and TP63. In these cases both integration in exon and intron sequences was detected. In addition, half of the integration sites are in close proximity to fragile sites.
Conclusions: In conclusion, this is the first study providing evidence for and a detailed analysis of HPV 16 integration sites in approximately half of HPV-positive OSCC. We were unable to identify viral integration in the remaining tumours by means of APOT and DIPS PCR, indicating that these tumours harbor episomal HPV copies and/or integration of complete episomes. Studies are ongoing to further examine HPV-driven tumorigenesis in both subgroups.

1330 Association between Stromal Myofibroblasts and Molecular, Clinical, and Histopathological Features of Premalignant and Malignant Lip Lesions

ML Spencer, P Zapata, A Martinez, N Riquelme, O Salvatori, IG Rojas. College of Dentistry, University of Concepcion, Concepcion, Chile.

Background: Tumor stroma composition is an important prognostic factor in several neoplasias. An increased density of stromal myofibroblasts is associated with higher tumor aggressiveness and mortality in oral squamous cell carcinoma (SCC). Lip SCC is a type of oral cancer characterized by altered $\mathrm{p} 53$ and COX-2 expression as well as by an activated stroma both in its premalignant and malignant stages, and includes increased angiogenesis, inflammatory infiltration and fibroblast density. Since myofibroblasts have not been characterized during lip carcinogenesis, the aim of this study was to assess myofibroblast density and its association with molecular and clinicopathological features of both the premalignant lip lesion, actinic cheilitis (AC) and lip SCC.

Design: Sixty two AC samples (11 with dysplasia) and 54 lip SCC samples were processed for immunohistochemical detection of myofibroblasts with the marker smooth muscle actin (SMA). Immunohistochemical detection of p53 and COX-2 was also performed. Samples were scored 1 if SMA+ cells corresponded only to blood vessels, 2 if SMA + myofibroblasts were $<50 \%$ of the stroma, and 3 if SMA+ myofibroblasts were $>50 \%$ of the stroma. Scores (based on intensity and extension) were also obtained for $\mathrm{p} 53$ and COX-2 expression at the epithelial or tumor compartments of each sample. Results: Myofibroblasts were significantly increased in lip SCC (55\%) as compared to AC (13\%) (P<0.0001, Fisher's Exact test). In lip SCC samples, 44\% had score 1, 24\% score 2 , and $31 \%$ score 3 . In AC samples, $86 \%$ had score 1 and $13 \%$ score 2 , with no score 3. Female patients with lip SCC showed higher stromal myofibroblast density than males $(\mathrm{P}<0.05)$. Myofibroblast density had no association with TNM stage or tumor differentiation, however, it was associated with epithelial dysplasia in $\mathrm{AC}(\mathrm{P}<0.05)$. Stromal myofibroblast density was also associated with $\mathrm{p} 53$ expression both in AC and lip SCC $(\mathrm{P}<0.0001$, t-test $)$, but no association was found with COX-2 expression. Conclusions: Myofibroblast density increases as lip cancer progresses, and it is associated with gender (higher in females) and p53 expression. This suggests that stromal myofibroblasts are important markers of malignancy during lip carcinogenesis. Supported by CONICYT Chile, grant FONDECYT 1090287.

1331 Clinicopathologic and Immunophenotypic Characterization of Six Dedifferentiated Acinic Cell Carcinomas

$J N$ Stall, JB McHugh. University of Michigan, Ann Arbor, MI

Background: Dedifferentiated acinic cell carcinoma (ACC) is a rare variant composed of low-grade (LG) ACC and a high-grade (HG) dedifferentiated component, either poorly differentiated adenocarcinoma or undifferentiated carcinoma. Given its aggressive clinical course, dedifferentiated ACC is important for surgical pathologists to recognize. Since only twenty cases have been reported and characterized to date, our goal was to describe the clinical, morphologic and immunophenotypic spectrum of six new cases.

Design: An institutional review from 1990-2011 for dedifferentiated ACC and highgrade carcinoma, not otherwise specified (NOS) identified six total cases. Clinical information and tissue were available for six and five cases, respectively. We evaluated the histologic features as well as immunophenotype using antibodies to CAM5.2, actin (SMA), S100, p63, p53, Ki-67, cyclinD1, $\beta$-catenin, estrogen receptor (ER), Her-2/neu, and androgen receptor (AR). Five LG ACC were stained for comparison.

Results: Median age was 55.5 y (range $47-70 \mathrm{y}$ ), with equal sex predilection and all occurred in the parotid. Half involved the facial nerve at presentation. Most (5/6) patients received total parotidectomy and LN dissection with adjuvant therapy (4/6). Distant metastasis occurred in $3 / 5$ and LN metastasis in $4 / 5$ cases over a median of $12.5 \mathrm{~m}$ (range 9-24 m). 4/5 died with a median survival of $2.8 \mathrm{y}$ (range $0.9-8.8 \mathrm{y}$ ) and one is alive with disease. All were composed of LG microcystic ACC and a HG component consisting of invasive lobules of undifferentiated cells. Cribriform growth was seen in $2 / 5$ and glands in $3 / 5$. All HG areas had cells with acinic differentiation and these were prominent in 4/5. Perineural invasion, high mitotic rate, atypical mitoses and comedonecrosis were each seen in $4 / 5$ cases. Compared to the LG ACC, the dedifferentiated components showed decreased S100 dendritic cells and CAM5.2 staining with increased cyclinD1, p53 and $\mathrm{Ki}-67$. All cases were positive for membranous $\beta$-catenin and all were negative for p63, S100, SMA, AR, ER and Her-2/neu.

Conclusions: Dedifferentiated ACC demonstrated an aggressive clinical course with frequent facial nerve involvement, metastases and death. No definitive immunophenotypic profile could be determined from this small cohort, but the increased cyclinD1, p53 and Ki-67 are consistent with a more aggressive neoplasm. Although some may resemble salivary duct carcinoma, the negative AR and Her-2/neu argue against this. Recognition of dedifferentiated ACC is important for surgical pathologists as more aggressive clinical management is warranted. 
1332 microRNA Expression in Archived FFPE Head and Neck Squamous Cell Carcinomas Utilizing Multiplex miRNA Expression Assays

LJ Tafe, TH Davis, MC Schwab, GJ Tsongalis. Dartmouth Hitchcock Medical Center, Lebanon, NH.

Background: Micro RNAs (miRNA) play a role in regulating the expression of genes that are important in cellular differentiation, proliferation, survival and cell death Multiple studies have established that many cancer types, including leukemias and solid tumors, exhibit distinct miRNA expression profiles that likely play a role in tumorigenesis. Limited studies have examined miRNA expression in head and neck squamous cell carcinomas (HNSCC). The aims of this study were twofold: first, to determine if FFPE is an acceptable sample type for the multiplex miRNA expression assays, and, second, to identify miRNA expression patterns that may be characteristic of HNSCC when compared to matched normal tissues.

Design: Total RNA was extracted and purified from 24 archived FFPE matched tumo and normal HNSCC (6 laryngeal, 6 oral tongue) using the Qiagen RNeasy FFPE kit Approximately 100ng of total RNA was run in a final reaction volume of 30ul using nanoString's nCounter miRNA Expression Assay Kit on the nCounter platform. This assay detects over 700 miRNAs in a multiplex format. The geometric mean of the top 150 expressing miRNAs was taken for normalization. The expression of miRNAs in tumor tissues was compared to the matched normal counterparts.

Results: Two of 24 samples (1 tumor, 1 normal) showed low counts, indicating possible degradation, therefore, a total of 10 sample pairs had analyzable results (4 laryngeal and 6 tongue). The tumors expressed a median of 147 miRNAs (range 82-435) and the normal tissues expressed a median of 133 miRNAs (range 107-224). Several patterns of expression were observed: miR-21 was upregulated in 7/10 cases (3-14 fold) including $5 / 6$ tongue tumors. MiR- 205 and miR-1274b were upregulated in 5/10 total cases (3-10 fold) and in 4/6 tongue tumors ( $3-4$ fold), respectively. MiR-451 and miR-125b showed downregulation in 6/10 (3-72 fold) and 5/10 (3-12 fold) total tumors, respectively. Conclusions: In this study, we have shown that the nCounter miRNA Expression Assay Kit can be successfully run on HNSCC FFPE archival tissue. Our finding of the upregulation of miR-21 in HNSCC are consistent with prior reports in the literature. In addition, we identified other miRNAs that may play a regulatory role in the tumorogenesis of HNSCC and that may have a tumor site specific profile (i.e. tongue vs. larynx) that lend themselves to further investigation.

\section{P53 Expression in Oropharyngeal Squamous Cell Carcinoma Is} Increased in HPV Positive Smokers

$M$ Tuluc, V Bar-Ad, Z Wang, J Curry. Jefferson University Hospital, Philadelphia, PA. Background: Patients with HPV-positive oropharyngeal carcinoma (OSCC) have a better prognosis than patients with HPV-negative OSCC. Differences in expression of cell cycle and survival proteins and p53 gene mutations have been identified between HPV-positive and HPV-negative OSCC. HPV E6 protein inactivates p53, therefore HPV positive OSCC have low expression of p53. In contrast, in chemical/toxin induced OSCC $\mathrm{p} 53$ is overexpressed and it is associated with poor prognosis and resistance to treatment. This study evaluates $\mathrm{p} 53$ expression in HPV positive and HPV negative OSCC patients with and without smoking history.

Design: 101 cases of OSCC, 65 p16 positive and 36 p16 negative patients were retrospectively collected. The two groups have been divided in nonsmokers, smokers and patients with remote smoking history (who quit smoking at least 3 years prior to diagnosis)

Immunohistochemistry stains for $\mathrm{p} 16$ and $\mathrm{p} 53$ were performed on all cases. The staining pattern for p 53 has been defined as low expression (less than $20 \%$ of the nuclei positive) or high expression (more than $20 \%$ nuclear staining)

Results: There were significant differences in $\mathrm{p} 53$ staining pattern between $\mathrm{p} 16$ positive and p16 negative patients. P16 negative smokers (current and remote smokers) had the highest percentage of $\mathrm{p} 53$ overexpression ( 38 and $40 \%$, respectively).

The highest percentage of $\mathrm{p} 53$ overexpression in the $\mathrm{p} 16$ positive patients was in the smokers group, $15 \%$ of cases.

In p16 positive nonsmokers only $4 \%$ of patients showed high p53 staining. p53 levels in nonsmokers, p16 positive and p16 negative was low (under $10 \%$ ).

Conclusions: In the p16 negative group, patients with current and remote smoking history showed the highest $\mathrm{p} 53$ expression. This is concordant with the literature data that suggests that $\mathrm{p} 53$ is overexpressed in non HPV related head and neck SCC. Our study brings strong evidence that in HPV positive patients with OSCC that are current smokers there is an increase in p53 expression, at a level that is intermediary between p16 positive nonsmokers and p16 negative smokers. We hypothesize that smoking partially reverses the effect of E6 HPV protein on p53 inactivation. The small percentage of HPV positive nonsmokers with high p53 expression suggests that in HPV OSCC other pathways, independent of HPV, may be involved in p53 inactivation, hypothesis that may carry therapeutic and prognostic implications.

1334 P16/Rb Pathway in Oropharyngeal Squamous Cell Carcinoma $M$ Tuluc, V Bar-Ad, D Cognetti. Jefferson University Hospital, Philadelphia, PA.

Background: HPV oncoproteins E6 and E7 interfere with crucial cellular mechanisms such as cell regulation and apoptosis. E6 disrupts the p53 pathway while E7 inactivates $\mathrm{Rb}$.

P16 activates Rb-dependent cell cycle arrest; in cells with functional Rb, elevated p16 is a potent inhibitor of cellular proliferation. HPV positive tumors which are characterized by loss of Rb function secondary to E7 oncoprotein activity, have high levels of p16. In consequence, HPV positive oropharyngeal squamous cell carcinoma (OSCC) is characterized by overexpression of p16 and low $\mathrm{Rb}$.
However, in many patients with OSCC, HPV is not the only carcinogen and superimposed chemical/toxin carcinogens (smoking and alcohol) often play an important role in tumor initiation and progression.

The objective of this study is to explore the p16/Rb pathway in patients with p16 positive and $\mathrm{p} 16$ negative OSCC.

Design: $\mathrm{Rb}$ and $\mathrm{p} 16$ expression was evaluated by immunohistochemistry in 66 cases of p16 positive and 36 cases of p16 negative OSCC. Smoking history was available in all cases.

$\mathrm{Rb}$ expression was assessed as low if $20 \%$ or less of tumor cells showed nuclear staining and high if nuclear staining was present in more than $20 \%$ of tumor cells.

p16 was considered positive if more than $50 \%$ of the tumor cells demonstrated cytoplasmic and nuclear staining.

Results: More than $50 \%$ of the 66 patients with p16 positive OSCC showed low Rb (59\% in nonsmokers, $73 \%$ in remote smokers and $62 \%$ in smokers). The remaining p16 positive patients had high $\mathrm{Rb}$ expression.

Among the 36 cases of p16 negative OSCC, $100 \%$ of smokers had high Rb expression, while in the nonsmoker and remote smoker groups only $20 \%$ of patients showed high $\mathrm{Rb}$ expression.

Conclusions: Low Rb expression in p16 positive OSCC is in agreement with the effect of HPV E7 oncoprotein on the $\mathrm{p} 16 / \mathrm{Rb}$ axis. However, approximately $30 \%$ of $\mathrm{p} 16$ positive patients had high $\mathrm{Rb}$ expression, irrespective of their smoking history. This may be due to differences in the degree of Rb inhibition by E7 oncoprotein, making the HPV action on the host cell more important than patients' smoking history.

High Rb expression in p16 negative OSCC supports the hypothesis that cell proliferation in HPV negative tumors may involve loss or silencing of the p16 tumor suppressor via a pathway independent of $\mathrm{Rb}$ activity.

\section{B7-H1 Expression Model for Immune Evasion in Human} Papillomavirus Associated Squamous Cell Carcinomas

OC Ukpo, WL Thorstad, JS Lewis. Washington University, Saint Louis, MO.

Background: Human papillomavirus (HPV) has been associated with oropharyngeal squamous cell carcinomas (OSCC). Persistent viral infection is postulated as the mechanism leading to carcinogenesis. It is known that immune evasive cells can provide an ideal niche for a virus. The B7-H1/PD-1 cosignaling pathway plays an important role in viral immune evasion by making CD8+ cytotoxic T-cells anergic. Tumor cell B7-H1 expression has also been associated with poorer survival in numerous carcinomas, but its role in HPV-related OSCCs is unknown. We hypothesized that HPV-related OSCCs express B7-H1 as a potential mechanism for HPV immune evasion.

Design: A tissue microarray of OSCC was utilized, for which HPV E6/E7 mRNA by in situ hybridization was previously performed. Immunohistochemistry was performed to detect B7-H1 on tumor cells, which was scored as positive or negative. Eithe membranous or cytoplasmic staining was considered positive. Screening was performed by two pathologists (OCU, JSL), both blinded to clinical information.

Results: B7-H1 was expressed in 82 of the 181 (45.8\%) cases. Tumors expressing B7-H1 were more likely to be HPV positive.

B7-H1 and HPV E6/E7 mRNA Expression

\begin{tabular}{|l|l|l||}
\hline \hline & B7-H1 Positive & B7-H1 Negative \\
\hline HPV mRNA Positive & 68 & 70 \\
\hline HPV mRNA Negative & 14 & 27 \\
\hline Total & 82 & 97 \\
\hline
\end{tabular}

p-value $=0.08$

B7-H1 showed a significant trend toward correlation with distant metastasis in the entire cohort $(\mathrm{OR}=2.85, \mathrm{p}=0.12)$, a strong trend in HPV negative cases $(\mathrm{OR}=7.01, \mathrm{p}=0.10)$, but no trend in the HPV positive cases $(\mathrm{OR}=1.56, \mathrm{p}=0.63)$. $\mathrm{B} 7-\mathrm{H} 1$ expression was not associated with recurrence in the entire cohort $(\mathrm{OR}=1.09, \mathrm{p}=0.80), \mathrm{HPV}$ positive $(\mathrm{OR}=$ $0.80, \mathrm{p}=0.69)$ or HPV negative $(\mathrm{OR}=2.02, \mathrm{p}=0.22)$ cases. $\mathrm{B} 7-\mathrm{H} 1$ was not associated with overall survival for the entire cohort $(\mathrm{RR}=0.91, \mathrm{p}=0.73)$, and HPV positive cases $(\mathrm{OR}=0.84, \mathrm{p}=0.64)$, but showed a trend toward poorer survival in HPV negative cases $(\mathrm{OR}=1.65, \mathrm{p}=0.38)$

Conclusions: $\mathrm{B} 7-\mathrm{H} 1$ is expressed in a significant subset of tumors with HPV mRNA viral transcript expression. This suggests that $\mathrm{B} 7-\mathrm{H} 1$ positive tumor cells may play a role in harboring persistent HPV infection. However, it does not appear to be associated with tumor recurrence or poorer survival, perhaps due to risk modification by the presence of HPV. Distal metastasis may be associated with B7-H1 expression, but a larger sample size is required.

1336 Molecular Characterization of 61 Cases of Follicular Adenomas and Carcinomas of the Thyroid

P Vielh, CRichon, G Meurice, B Job, L Lacroix, VMarthy, NMotte, A Valent, B Caillou, A Al Ghuzlan, J-M Bidart, A El Naggar, V Lazar, P Dessen, $M$ Schlumberger. Institut Gustave Roussy, Villejuif, France; MD Anderson Cancer Center, Houston.

Background: Although fine needle aspiration cytology (FNAC) is an efficient method for diagnosing the benign or malignant nature of thyroid nodules, distinction between follicular adenomas and carcinomas may still represent a difficult task. Identification of distinct genomic alterations between these two groups of tumors, potentially applicable to cytological specimens, should be therefore of major interest.

Design: A series of 61 cases consisting of 31 cases of follicular adenomas and of 30 cases of follicular carcinomas were characterized by molecular methods: mutations in the RAS ( $H, K$ and $N$ ), BRAF, CTNNBI and PIK3CA genes were assessed by sequencing, PAX8/PPARg and RET/PTC1 translocations by RT-PCR, and microRNA (miRNA) expression using $8 \times 15 \mathrm{~K}$ microarrays.

Results: Out of the 61 patients, 35 were female and 26 were male with a median age of 59 ( 21 to 86$)$ and 58 ( 28 to 83 ) years, respectively. Median size of follicular adenomas was 3 (1 to 5.5$) \mathrm{cm}$ and histological subtypes were microfollicular in $6(19 \%)$ cases, 
macrofollicular in $9(29 \%)$ cases and of mixed subtype in $16(52 \%)$ cases. Mean size of follicular carcinomas was 3.7 ( 1 to 15 ) $\mathrm{cm}$ and subtypes were microinvasive in 18 $(60 \%)$ cases and widely invasive in $12(40 \%)$ cases. Mutations $(\mathrm{n}=1)$ and translocations $(\mathrm{n}=0)$ were rarely found in adenomas as compared with follicular carcinomas $(\mathrm{n}=11$ and $n=3$, respectively).

After data processing, $281 \mathrm{miRNA}$ were validated for further analysis. An unsupervised clustering of 57 tumor samples (adenomas and carcinomas) shows two well distinct clusters: the first one covers 26 out of 28 carcinomas (93\%) whereas the second one covers 28 out of 29 adenomas $(97 \%)$. The differential analysis of carcinomas versus adenomas raised 20 miRNA: 19 were over-expressed in carcinomas, and one in adenoma. Further analysis of these 20 miRNA allows us to identify a signature composed of 10 miRNAsdistinguishing adenomas from carcinomas.

Conclusions: In conclusion, follicular lesions of the thyroid are clearly differentiated by the study of a small subset of miRNAs. Further studies are needed to verify these data before being used as an adjunct for helping the differential diagnosis between follicular adenomas and follicular carcinoma on cytological material obtained by FNAC, and to explore their functional significance.

\section{A Cost-Effectiveness Analysis of Thyroid Frozen Sections} SM Voss, HS Crist, H Mani. PSMSHMC, Hershey, PA.

Background: Routine use of frozen sections (FS) during thyroid surgery continues to be controversial. Studies have advocated the use of FS only in cases labeled suspicious or indeterminate on cytology (FNAC). We performed a cost-effectiveness analysis of thyroid FS use to evaluate the above strategy.

Design: A retrospective analysis of the pathology database was performed to identify thyroid FS over a 7-year period. Results of FNAC, FS and final pathology (FHP) were collated and data analyzed. Cost effectiveness was analyzed based on medicare reimbursement rates for FNAC (\$134), FS (\$89), surgery (lobectomy \$767, thyroidectomy \$970) and FHP (\$219).

Results: 152 thyroid FS were carried out in the study period. FS diagnoses correlated with FHP in $128(84 \%)$ cases (33 malignant and 95 benign). FS had a sensitivity of $64 \%$ and specificity of $94 \% .115$ cases had satisfactory FNAC, with a sensitivity of $73 \%$ and specificity of $64 \%$. Of the 33 cases diagnosed as malignant on FS, 17 had a diagnosis of malignancy on FNAC and FS did not add to the surgical strategy in these cases. FS changed the surgical strategy in $20(13 \%)$ cases that were not malignant by FNAC. Diagnoses on FS and FHP in non-malignant FNAC categories are shown in Table 1. False negative diagnoses on FS included 11 papillary carcinomas ( 8 micro and 3 follicular variant) and 3 minimally invasive follicular carcinoma.

Table

\begin{tabular}{|c|c|c|}
\hline FNAC category & FS & FHP \\
\hline Benign & 25 benign, $6 \mathrm{FN}, 3 \mathrm{PTC}$ & 27 benign, 3 PTC, $4 \mathrm{uCa}$ \\
\hline FN & 19 benign, $23 \mathrm{FN}, 3 \mathrm{PTC}$ & 39 benign, $3 \mathrm{PTC}, 2 \mathrm{uCa}, 2 \mathrm{FCa}$ \\
\hline Suspicious & 4 benign, 5 FN, 6 PTC & 8 benign, 7 PTC \\
\hline
\end{tabular}

FN-follicular neoplasm, PTC-papillary thyroid carcinoma, uCa-papillary microcarcinoma, FCaFollicular carcinoma

Based on our data, if FS had been performed in all the 125 cases that did not have a preoperative FNAC diagnosis of malignancy, and if surgical strategy of lobectomy vs. total thyroidectomy had been based on FS diagnosis in these cases, the total cost would be $\$ 85628$. On the other hand, if FS were performed only in the 15 cases with a diagnosis of suspicious/indeterminate on FNAC, there would be a saving of $\$ 9790$ on FS costs. However, this strategy would have missed the 6 malignancies that were diagnosed on FS in other FNAC categories (Table 1). These 6 would have required completion lobectomy at a second sitting (cost \$5916), reducing the savings to \$3714. It is likely that other costs of second hospitalization and lost man-hours at work would outweigh these limited savings.

Conclusions: Intraoperative FS does not yield additional information in cases with an FNAC diagnosis of malignancy. FS is both cost-effective and impacts surgical strategies in cases with non-malignant FNAC diagnoses.

1338 Spindle Cell Carcinomas of the Head and Neck Are Rarely Associated with Human Papillomavirus

RF Watson, RD Chernock, H Wang, X-JMa, Y Luo, SK El-Mofty, JS Lewis. Washington University School of Medicine, Saint Louis, MO; Advanced Cell Diagnostics, Inc., Hayward, CA.

Background: Spindle cell carcinoma ( $\mathrm{SpCC}$ ) is a rare variant of squamous cell carcinoma characterized by spindled or pleomorphic cells which appear to be a true sarcoma but are actually of epithelial origin. Recent studies have shown that certain variants of head and neck squamous cell carcinoma are human papillomavirus (HPV) -related and are associated with more favorable outcomes. The purpose of this study was to determine the prevalence of HPV in SpCC and to explore the effect of HPV status on patient outcomes.

Design: Cases with a diagnosis of SpCC, sarcomatoid carcinoma, or SCC with a spindle cell component were retrieved from pathology department files. The diagnosis required a biphasic lesion with a clearly recognizable squamous neoplastic component or alternatively a pure spindle cell or pleomorphic tumor with documented immunohistochemical positivity for epithelial cell markers. The presence of transcriptionally-active HPV was determined by $\mathrm{p} 16$ immunohistochemistry and both DNA and RNA in situ hybridization (ISH) for high risk HPV E6 and E7 transcripts. We also reviewed the medical records and follow up information for all patients.

Results: Of 32 cases, $5(15.6 \%)$ were from the oropharynx, $12(37.5 \%)$ from the oral cavity, $1(3.1 \%)$ from the maxillary sinus, and $14(43.8 \%)$ from the larynx or hypopharynx. The average stage at presentation of patients with oropharyngeal SpCC was higher when compared to other sites but there were too few patients to determine statistical significance. None of the oropharyngeal SpCCs were positive for $\mathrm{p} 16$ or for high risk HPV by DNA or RNA ISH. Only one of the 27 non-oropharyngeal SpCC (oral cavity) was HPV positive by both DNA and RNA ISH. It also stained for p16. Another SpCC from the larynx was HPV positive by both DNA and RNA ISH in both components, but it did not stain for p16. A third case from the maxillary sinus was positive for p16 in the conventional SCC component but negative for HPV by ISH. Both HPV positive patients presented at high stage (IV), and they died with disease at 22 and 9 months after diagnosis, respectively.

Conclusions: The majority of SpCCs of the head and neck, including the oropharynx, are not HPV related, and for the rare HPV-related cases, the presence of HPV does not appear to provide any prognostic benefit, although the number of cases is insufficient to make any definitive conclusions.

1339 Identification of Oncogenic Mutations in Adenoid Cystic Carcinoma $D$ Wetterskog, DN Rodrigues, $K$ Fritshie, MB Lambros, $S$ Shousha, Z Gatalica, B Weigelt, A Vincent-Salomon, G Stenman, B Rubin, JS Reis-Filho. The Institute of Cancer Research, London, United Kingdom; Cleveland Clinic, Cleveland; Charing Cross Hospital, London, United Kingdom; Creighton University School of Medicine, Omaha, United Kingdom; Cancer Research UK London Research Institute, London, United Kingdom; Institut Curie, Paris, France; Sahlgrenska Cancer Center, Gothenburg, Sweden.

Background: Adenoid cystic carcinomas (AdCCs) are malignant tumours that affect multiple anatomical sites, including the salivary gland, lung and breast. $30 \%-90 \%$ of these tumours harbour the $M Y B-N F I B$ fusion gene, caused by a $\mathrm{t}(6: 9)$ translocation, which results in MYB overexpression. Salivary gland and breast AdCCs have distinct clinical behaviours; while salivary gland AdCCs are prone to distant relapses, patients with breast AdCCs have an excellent prognosis. The aim of this study was to investigate the presence of mutations in known oncogenes in AdCCs.

Design: Twelve $M Y B-N F I B$-positive and one $M Y B-N F I B$-negative breast AdCCs were microdissected to ensure $>90 \%$ of tumour cell content, and DNA was extracted. The prevalence of hot-spot mutations in 19 known oncogenes was assessed using the Sequenom OncoCarta Panel v1.0, and validated with Sanger sequencing. Genes identified as mutated in any of the cases, and genes related to the same canonical pathways as those of the mutated genes, were sequenced in a separate cohort of 56 AdCCs (2 breast, 4 lung, 48 salivary gland, 1 pancreatic and 1 vaginal wall) using Sanger sequencing.

Results: Using Sequenom MassArray we identified BRAF mutations (i.e. V600E and $G 464 E$ ) in 2/13 breast AdCCs (15\%), which were confirmed by Sanger sequencing. Both cases harbouring $B R A F$ mutations were $M Y B-N F I B$ fusion-positive. No hotspot mutations in $B R A F$ and other genes of the RAS-RAF pathway, namely NRAS, KRAS and $H R A S$, and also in $K I T$, a gene previously reported to be mutated in AdCCs, were identified in a separate cohort of 56 AdCCs by Sanger sequencing.

Conclusions: A subset of breast AdCCs, but not AdCCs from other anatomical sites, harbour activating $B R A F$ mutations, albeit of low prevalence. These data provide evidence to suggest that the RAS-RAF pathway may be activated in tumours of indolent clinical outcome, such as breast AdCCs. Additional studies testing whether $B R A F$ mutations constitute a 'driver' event in $M Y B$-NFIB-positive AdCCs are warranted. Mutations in the genes assessed by Oncocarta v1.0 are unlikely to account for the differences in clinical behaviour between AdCCs of different anatomical sites. Finally, the lack of KIT mutations in our cohort suggests that KIT overexpression is unlikely to constitute a useful therapeutic target for AdCCs.

1340 African Americans with Oropharyngeal Carcinoma: Decreased Transcriptionally Active High-Risk Human Papillomavirus Contributes to Poorer Survival

$J X u, Q D a i, T$ Isayeva, S Hebert-Magee, M Brandwein-Gensler. University of Alabama at Birmingham, Birmingham, AL.

Background: High-risk Human Papillomavirus (HPV), mostly HPV16 (90\%), followed by HPV 18 (5\%), promote at least $50 \%$ of oropharyngeal cancers (OPC). Recent studies confirm the improved outcome for HPV+ OPC patients, compared to HPV- counterparts. Settle et al recently demonstrated a significantly poorer survival for African Americans (AA) with OPC, as compared to Caucasians; they attributed this to differences in HPV infection rates. However their study assessed only HPV DNA, rather than confirmed the viral transcriptional status.

Design: We retrospectively identified 102 consecutive patients with OPC. Data on demographics (age, gender, race, stage, treatment, alcohol (Yes/No), smoking exposure (Yes/No) and outcome (progression-free survival (PFS), disease-specific survival (DSS)) were collected. RNA was extracted from archival tissue blocks of interest and reverse transcription was performed. Nested quantitative real-time PCR was performed with primers to HPV16 (E6 (including E6*)/E7 and HPV18 (E6/E7). Positive and negative controls (SiHa, HeLa, HPV16+ OPC, and pancreatic carcinoma, water, respectively) were included in all reactions. Tumors were classified as HPV+ if both concordant E6 and E7 transcripts were present. The data were analyzed by two-tailed Fisher's exact test and Kaplan Meier (KM) curves.

Results: This cohort of 102 patients included 78 Caucasians and 24 African Americans (AA) with OPC. Among Caucasians: 54\% (42/78) were HPV16+, 12\% (9/78) were HPV $18+$, and 6\% (5/78) were double positive. Among AA: $29 \%(7 / 24)$ were HPV16+, $17 \%$ (4/24) were HPV18+, and none were double positive. OPC from Caucasians were significantly associated with HPV16 $(\mathrm{p}=0.039)$. KM curves revealed decreased time to PFS and DSS for AA patients ( $\mathrm{p}=0.007$, and $\mathrm{p}=0.001$, respectively) compared to Caucasians. Improved PFS and DSS was associated with HPV16+ OPC (all races, $p$ $=0.007$, and $\mathrm{p}=0.006$, respectively). No associations were seen for HPV18. Figure 1 demonstrates disease-specific survival per race and HPV16 status $(\mathrm{p}=0.0010)$. 


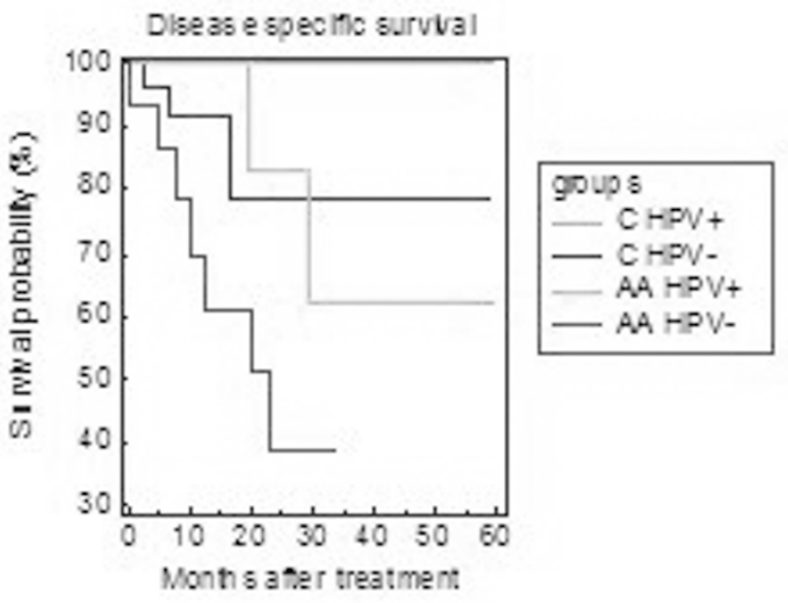

Conclusions: Our data confirm that transcriptionally active HPV16 imparts an improved prognosis for Caucasians with OPC. OPC in AA patients are significantly less likely to be HPV16-mediated.

\section{Expression of PAX2 and Renal Cell Carcinoma Antigen in Mucoepidermoid Carcinoma}

$B X u$, RT Cheney, A Omilian, Y Liu, CMorrison. Roswell Park Cancer Institute, Buffalo, NY; State University of New York at Buffalo, Buffalo, NY.

Background: Mucoepidermoid carcinoma (MEC) is the most common malignant tumor of salivary origin. It is a heterogeneous neoplasm in which clear cells accoun about $10-60 \%$ in most of MEC. Clear cell renal cell carcinoma (CCRCC) is the most common metastatic clear cell tumor in head and neck. The distinction between MEC with clear cells (CMEC) and metastatic CCRCC can be challenge in a small biopsy specimen. The aim of this study is to evaluate the utility of expression of PAX2 and RCCma between CMEC and metastatic CCRCC in a clinical setting.

Design: Twenty-three cases of MEC and ninty-four cases of metastatic RCC were identified from Roswell Park Cancer Institute (RPCI) pathology archives from 2000 to 2010 for TMA construction. Among MECs, 11 are of major salivary gland origin (parotid and submaxillary glands), 11 of minor salivary gland origin, and 1 involving a cervical lymph node. Immunostains on TMA sections were carried out according to manufacturer's protocols. This study was approved by Institution Review Board at RPCI Results: The 23 cases of MEC were classified according to the criteria by WHO as low grade (14 cases, $61 \%$ ), intermediate grade (5 case, $22 \%$ ) and high grade ( 4 case, $17 \%$ ). Eleven cases (48\%) show variable clear cell component (CMEC), ranging from $20-60 \%$ of the tumor. Majority CMEC were low grade ( $82 \%)$. RCCma was positive in $3 / 11$ CMEC $(13 \%)$ with variable degree of membranous and cytoplasmic staining. All of these cases were low grade. Positive immunoreactivity for PAX2 was found in 21 cases $(91 \%)$ showing diffuse cytoplasmic reactivity. However, no cases showed nuclear staining that is specific for PAX2. In metatstaic RCC, the nuclear staining for PAX2 was demonstrated in 21 of 94 cases $(22 \%)$ and the membranous staining for RCCma was found in 19 cases $(20 \%)$. In six cases of metastatic RCC to the head and neck, only two cases were positive either for PAX2 or RCCma. Three cases that metastasize to salivary glands and thyroid were negative for PAX2 and RCCma.

Conclusions: In this study, expression of RCCma was found in a small subset of MEC. These findings have not been reported previously. Caution should be made when one interprets positive RCCma and PAX2 immunostaining results on work-up for differential diagnosis between CMEC and metastatic CCRCC. Clinicopathologic correlation combined with histomorphology and a panel of immunohistochemical markers are essential to render correct diagnosis.

1342 MYB Overexpression Associated with Advanced Stage of Adenoid Cystic Carcinoma and Together with TTF-1 Identifying Rare Lung Primary Subtype

L Zhang, SYT Loo, D Shimizu, SA Honda, Y Cao, A Powers, M Carbone. University of Hawaii, Honolulu, HI.

Background: $\mathrm{t}(6,9)(\mathrm{q} 22-23 ; \mathrm{p} 23-24)$ MYB-NFIB reciprocal translocation has been recently linked to adenoid cystic carcinoma. However the relationship between the resultant MYB overexpression and clinical outcome is not clear. Besides, lung metastasis versus lung primary adenoid cystic carcinoma is occasionally a pathological diagnostic challenge.

Design: To address these questions, we examined MYB and TTF-1 protein expression in a consecutive case cohort of adenoid cystic carcinoma including 41 formalin fixed paraffin embedded specimens biopsied or surgically resected from 34 patients.

Results: The overall positivity of immunoperoxidase for MYB is 31/41 (76\%). In the salivary, nasosinal, orbital and supraglottic locations, MYB is overexpress in 14/15 (93\%) cases which have local recurrence, distal metastasis or TNM stage III/IV; $7 / 12(58 \%)$ cases which are TNM stage I/II or of no recurrence for 7-12 years. The performances of MYB in other anatomic sites are: lung peripheral primary $5 / 5(100 \%)$; lung metastasis $3 / 3(100 \%)$; liver metastasis $1 / 1$, endotracheal $1 / 2(50 \%)$, thyroid $0 / 1$ and breast $0 / 1$. In addition, TTF- 1 is highly specific for adenoid cystic carcinoma of lung peripheral primary. TTF-1 is positive only in lung primary lesion $5 / 5(100 \%)$, negative for lung metastasis $0 / 3(0 \%)$, tracheal/thyroid origin $0 / 3(0 \%)$, and all the adenoid cystic carcinoma otherwise located $0 / 30(0 \%)$.

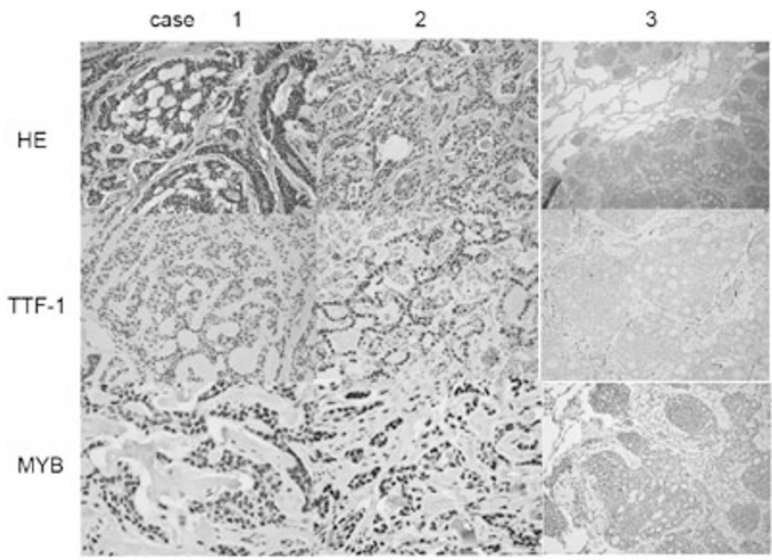

Fig 1. Representative immunostaining of 3 adenoid cystic carcinoma cases: 1. nasosinal primary: 2 . right lower lobe peripheral lung primary: 3 , right lung metastatic. Case $1 \& 2$ are from the same patient who has had 2 primary ad. enoid cystic carcinomas occuring 12 years apart. The TTF-1 staining in case 3 showed entrapped alveolar cells with tumor negative staining. All 3 cases are positive for MYB.

Conclusions: It seems that MYB overexpression is associated to advanced TNM stage, local recurrence and distant metastasis of adenoid cystic carcinoma of head and neck origin. TTF-1 is useful distinguishing lung primary peripheral subtype from metastatic adenoid cystic carcinoma.

1343 Activation of C-Jun N-Terminal Kinase Is Associated with Histological Grade of Mucoepidermoid Carcinoma

$H$ Zhong, A Omilian, C Morrison, B Xu. Roswell Park Cancer Institute, Buffalo, NY. Background: Mucoepidermoid carcinoma (MEC) is the most common malignant tumor of the salivary gland. Previous studies demonstrate that CTRC1-MAML2 fusion transcript, resulting from $\mathrm{t}(11: 19)$ translocation, is present in more than half of MECs. This translocation is associated with lower histologic grades and improved survival. In cultured cell lines, CTRC1 acts as a potent and indispensable modulator for activated protein 1 (AP1) transcriptional function which leads to anti-proliferation and proapoptosis. The intracellular signaling pathway mediating activation of AP1, however, has not been illustrated in human tissue of MEC. In this study, we investigate the activation of c-Jun N-terminal kinase (JNK) in human MEC by immunohistochemistry on sections of tissue microarray (TMA).

Design: Twenty-two cases of MECs, untreated before surgery, were identified at our institution from 2000 to 2010 . There were 11 of major salivary gland origin and 11 of minor salivary gland origin. Tumors were graded as low, intermediate and high grade according to WHO criteria. Immunohistochemical studies were performed on sections of TMA by using a mouse monoclonal antibody against phosphorylated Th 183 and Tyr 185 of JNK (pJNK) of human origin. Activation of JNK was determined by nuclear staining of $\mathrm{pJNK}$. Scoring of immunostain intensity was based on our previously published method.

Results: Positive nuclear staining of pJNK was demonstrated in 12 out of 13 low grade $(92.3 \%), 2$ of 5 intermediate grade $(40.0 \%)$ and 2 of 4 high grade $(50.0 \%)$ tumors. In addition, the percentage of $\mathrm{pJNK}$ positive tumors was significantly higher in low grade MECs in comparison with those in intermediate and high grade tumors (Pearson's Chi-Square test, $p=0.013$ ).

Conclusions: Activation of JNK is associated with tumor cell differentiation in MEC. These results explain previous findings that $\mathrm{t}(11: 19)$ translocation resulting the fusion transcript of CTRC1-MAML2 is present in majority of low grade MECs. Our future studies will be directed to explore the correlation between $\mathrm{t}(11 ; 19)$ translocation and activation of JNK in these MECs.

\section{ALDH1 Expression and Its Significance in Adenoid Cystic} Carcinoma of Salivary Glands

JH Zhou, EY Hanna, D Roberts, RS Weber, D Bell. MD Anderson Cancer Center, Houston.

Background: ALDH1 has been used as a marker for normal as well as cancer stem cells in several tissue types. Several investigators have also found it to be a prognostic/ predictive marker for carcinomas of the breast, lung, pancreas, bladder and etc. A recent study of adenoid cystic carcinoma (AdCC) using Aldeflour assay showed that cells with high level ALDH1 had higher tumorigenic, invasive and metastatic abilities. Currently there has been no report on immunohistochemical expression of ALDH1 in adenoid cystic carcinoma. In this study we examine the expression of ALDH1 in 216 adenoid cystic carcinomas using tissue micro-array and immunohistochemistry and its correlation with survival in a 12 year follow up cohort.

Design: Archival paraffin blocks of adenoid cystic carcinomas at the University of Texas, MD Anderson Cancer Center between 1988 and 2006 were used for this study with the approval of IRB. The tissue microarray was constructed using two $1.0 \mathrm{~mm}$ diameter cores of spatially different areas of representative tumor from each paraffin block. Normal salivary gland and splenic tissue was used as control. Immunohistochemical 
expression of ALDH1 was analyzed using mouse monoclonal ALDH1 antibody (BD Biosciences, San Jose, CA). Correlations between ALDH1 expression and clinical and histological parameters were assessed by Pearson's Chi-square and M-L Chi-square tests. Survival curves were generated using the Kaplan-Meier method and statistical differences by log rank test.

Results: Majority of the tumors (116, 63\%) showed stromal staining only, 21 (11\%) tumors showed both epithelial and stromal expression, 47 (26\%) tumors did not show either epithelial or stromal staining. The normal salivary gland showed epithelial expression only. Statistical analyses did not show any correlation between tumor pattern, tumor size, the presence of perineural invasion and the patterns of ALDH1 expression. The survival analysis using Kaplan-Meier method and log rank test did not show any significant differences among the three patterns of ALDH1 expression with survival. Conclusions: Other unknown factors, besides cancer stem cells, may play important roles in tumorigenesis, cell differentiation and tumor progression.

\section{Hematopathology}

1345 Characterization of Tissue Findings in Bone Marrow with Small Monoclonal B-Cell Populations

A Abdul-Nabi, L Peterson, B Nelson. Northwestern University, Chicago, IL.

Background: Flow cytometry immunophenotyping (FC) allows detection of small populations of CLL type or CD5- monotypic B-cells (MBCs) in the blood. When MBCs are not associated with overt clinical disease or tissue involvement, and are $<5000 / \mathrm{uL}$, the term monoclonal B-lymphocytosis (MBL) is used. Most studies have focused on PB MBL, but low levels of MBCs are also found in BM. In this study, we evaluated $\mathrm{BM}$ pathology and clinical setting when small MBC populations similar to MBL were detected in the aspirate by flow cytometry.

Design: BMs performed between 01/1999 and 12/2010 that showed MBCs $<5 \%$ of total events by $4-8$ color flow cytometry were selected for review. In BM biopsies done for lymphoma staging, only small clones with a phenotype different from the original lymphoma were included PB lymphocyte counts were $<5000 / \mathrm{uL}$. History and physical findings were retrieved from the EMR. H\&E stained BM sections and immunostains (IHC) for CD3, CD20 or PAX5 or CD79a were reviewed.

Results:

\begin{tabular}{|c|c|c|c|c|c|c|c|}
\hline \multirow{2}{*}{\begin{tabular}{||l||}
$\begin{array}{l}\text { Clone Type (no. of } \\
\text { cases) }\end{array}$ \\
\\
\end{tabular}} & \multicolumn{4}{|c|}{ Reason for BM } & \multicolumn{3}{|l|}{$\mathrm{H} \& \mathrm{E} / \mathrm{IHC}$} \\
\hline & $\begin{array}{l}\text { Lymphoma } \\
\text { staging }\end{array}$ & AML & $\begin{array}{l}\text { Plasma cell } \\
\text { dyscrasia }\end{array}$ & Cytopenias & $\begin{array}{l}\text { ymphoid } \\
\text { aggregates } \\
\text { (LA) }\end{array}$ & $\begin{array}{l}\text { B-cell rich } \\
\text { LA }\end{array}$ & $\begin{array}{l}\text { T-cell } \\
\text { rich } \\
\text { LA }\end{array}$ \\
\hline CLL (11/24) & 6 & 2 & 1 & 2 & $5 / 11$ & 3 & 2 \\
\hline None CLL (13/24) & 6 & 0 & 4 & 3 & $4 / 13$ & 1 & 3 \\
\hline
\end{tabular}

The majority of cases with either CLL or non-CLL type B-cell clones did not exhibit lymphoid infiltrates in the BM biopies. The cases that did have LA's were not considered diagnostic for lymphoma; all represented $<5 \%$ of cellularity and were non- paratrabecular; 5 were $\mathrm{T}$ cell rich and $4 \mathrm{~B}$ cell rich and were insufficient to established lymphoma.

Conclusions: In this study, small MBC clones were found in BM and were of both CLL type and non-CLL (CD5-) but the CD5- phenotype predominated, in contrast to those reported in the blood. Most BM biopsies with either type MBC had no lymphoid aggregates or no overt morphologic or IHC evidence for lymphoma. Recognition of these small B cell clones are important in evaluating BM biopsies for lymphoma as their presence, similar to $\mathrm{PB}$, may not be equivalent to lymphoma.

\section{Decreased Hematopoiesis in miR29ab1 Deficient Mice}

$R$ Aboomar, $S$ Saridakis, $R$ Santhanam, JR O'Rourke, F Racke, $R$ Garzon, EN Olson, $C M$ Croce, $S$ Costinean. The Ohio State University, Columbus; University of Texas Southwestern Medical Center, Dallas.

Background: MicroRNAs (miRs) are small noncoding RNAs that play a major role in the gene posttranscriptional regulation. Since their discovery they have been linked to numerous physiological and pathological processes. In 2002 miR15 was found downregulated in $65 \%$ of analyzed chronic lymphocytic leukemia cases. Later, miR $29 \mathrm{~b}$ was found downmodulated in acute myeloid leukemia.

Design: We decided to investigate the consequences of miR $29 \mathrm{~b}$ downregulation in genetically modified mice. We used cre-loxP technology to generate conditional knockouts (ko) with general deletion. We deleted separately the miR29ab1 and miR29b2 clusters and we crossed the two types of ko's in order to obtain the double $\mathrm{ko}, \mathrm{miR} 29 \mathrm{ab} 1 / \mathrm{b} 2 \mathrm{c}$ with complete deletion of miR29b. The mice were followed for two years. Mice were analyzed histologically. Cell blood counts were done periodically. Flow cytometry, colony forming assays and bone marrow transplantation were used to assess the hematopoiesis.

Results: The miR29ab1 ko's were normal at birth except for a skewed Mendelian ratio for the homozygous ( $10 \%$ percentage of born homozygous instead of $25 \%$ expected percentage). The homozygous have half the lifespan compared with wild type counterparts. The histology of ko spleens revealed a global decrease in hematopoiesis, especially in the myeloid lineage (half the levels of wild type mice). Colony forming assays performed at different ages confirmed a marked decrease of hematopoietic stem cells. Flow cytometry showed a dramatic increase of the c-kit positive population in the bone marrow of the ko animals. Mir29b2c did not exhibit any of these changes indicating that the alterations are miR29ab1 specific. We are now conducting bone marrow transplants to ascertain the diminished capacity of miR29a ko's to repopulate the bone marrow in irradiated mice. Of interest, mir29 double ko mice have a short life span, of only 1 month \pm 1 week.
Conclusions: Based on the present results we conclude that miR29ab1 ko's have decreased hematopoietic stem cell population compared to the wild types and that miR29ab1 might have an important role in the maintenance of this cell population. Also, miR29 genes might regulate immunity and life span, since both miR29ab1 and miR29ab1/b2c ko's seem to have markedly decreased life spans.

1347 The Majority of Immunohistochemically BCL2 Negative FL Grade I/II Carry A t $(14 ; 18)$ with Mutations in Exon 1 of the BCL2 Gene and Can Be Identified with the BCL2 E17 Antibody

P Adam, R Baumann, I Bonzheim, F Fend, L Quintanilla-Martinez. Eberhard-KarlsUniversity, Tubingen, Baden-Wurttemberg, Germany.

Background: Follicular lymphoma (FL) is characterized by the chromosomal translocation $\mathrm{t}(14 ; 18)(\mathrm{q} 32 ; \mathrm{q} 21)$ resulting in constitutional overexpression of the antiapoptotic protein BCL2. However, in $10-15 \%$ of FL grade I/II immunohistochemical (IHC) staining for BCL2 remains negative. The aims of this study were 1) to investigate the incidence of IHC BCL2 negative FL grade I/II in our series, 2)To analyze the BCL2 IHC negative FL with the new BCL2 antibody (clone E17) 3) To perform FISH analysis for the $t(14 ; 18)$ and 4$)$ to elucidate the molecular mechanism of BCL2 negativity.

Design: FL grade I/II diagnosed between $01 / 2005$ and 08/2011 at the Institute of Pathology of the University of Tübingen, Germany were included in the study. All cases were stained with the standard BCL2 antibody (clone 100D5; DCS). All BCL2 negative cases were subsequently stained with the BCL2 antibody, clone E17 (Zytomed) and analyzed by FISH using a $B C L 2$ break-apart probe (LSI $B C L 2$ BAP, Vysis). Exon 1 of the $B C L 2$ gene, where the epitope of the standard BCL2 antibody resides was amplified and sequenced.

Results: Of the 240 cases of FL grade I/II identified, 23 cases (9.6\%) were negative with the standard BCL2 antibody. Of these, 13 cases (57\%) were positive for the E17 antibody and 10 cases $(43 \%)$ remained negative. FISH analysis demonstrated a BCL2 break, indicative of a $(14 ; 18)$ in all E17 positive cases, whereas the E17 negative cases showed no $B C L 2$ alterations. Two of the $\mathrm{E} 17$ negative cases carried a $B C L 6 / I G H$ translocation. Mutation analysis of $B C L 2$ exon 1 revealed point mutations resulting in amino acid substitutions in all 9 analyzable E17 positive cases with a hot spot around codon 144 . Conclusions: 1) The incidence of IHC negative FL grade I/II in our series is comparable to published data. 2) The E17 antibody identifies the majority of BCL2 "negative" FL grade I/II and correlates with the presence of the $t(14 ; 18)$. 3) The negativity with the standard BCL2 antibody is due to point mutations in exon 1 of the BCL2 gene where the epitope of the antibody resides. 4)The molecular pathogenesis of the BCL2 (E17), $\mathrm{t}(14 ; 18)$ negative FL grade I/II remains to be determined.

1348 Normal Splenic Lymphoid Subsets Mimic Aberrant Antigen Expression

N Aggarwal, J Fischer, SH Swerdlow, FE Craig. University of Pittsburgh School of Medicine, Pittsburgh, PA.

Background: Flow cytometry (FC) assists in the diagnosis of lymphoma through identification of aberrant antigen expression. However, normal lymphoid subsets with less well-recognized phenotypes can mimic lymphoma. This study characterizes lymphoid subsets in normal spleen using 8-color FC.

Design: 20 spleens removed for traumatic rupture, with cell viability $>50 \%$, were analyzed within 24 hrs with the following FC combinations: CD16\&57/CD7/CD4/ $\mathrm{CD} 2 / \mathrm{CD} 56 / \mathrm{CD} 3 / \mathrm{CD} 5 / \mathrm{CD} 8 ; \mathrm{K} / \lambda / \mathrm{CD} 5 / \mathrm{CD} 19 / \mathrm{CD} 10 / \mathrm{CD} 38 / \mathrm{CD} 20 / \mathrm{CD} 45 ; \alpha \beta / \gamma \delta / \mathrm{CD} 3 /$ CD25 and CD14/CD13\&33/CD45/CD34.

Results: This study reveals the normal variation in splenic lymphoid subsets (Table) and demonstrates some subsets with phenotypes that have been associated with lymphoid neoplasms.

Well recognized lymphoma-associated phenotypes identified in this study include CD7- T-cells and CD5+ B-cells.

Less-well recognized phenotypes encountered in all cases, except as indicated, include: CD5- to dim+ CD8+ T-cells, CD3+ bright (br.) $\gamma \delta$ T-cells (15/20), CD5- $\gamma \delta$ T-cells (Fig. 1a), CD2-NK-cells and CD7+dim NK-cells (Fig 1b). The latter two NK-cell phenotypes were seen more often in the mature subtype $(\mathrm{p}<0.01)$.

\begin{tabular}{|c|c|c|c|}
\hline \multirow{2}{*}{ Population (Parent) } & \multicolumn{3}{|c|}{ \% of Parent Indicated } \\
\hline & Median & Min & Max \\
\hline T cells (Lymphoid cells) & 34 & 21 & 55 \\
\hline CD4+ (T cells) & 56 & 37 & 78 \\
\hline CD8+ $(\mathrm{T}$ cells $)$ & 33 & 13 & 46 \\
\hline CD4/CD8 ratio & 1.7 & 0.9 & 6 \\
\hline CD4+ CD8+ (T cells $)$ & 3 & 1 & 12 \\
\hline CD4- CD8- (T cells) & 7 & 1 & 19 \\
\hline$\alpha \beta+(T$ cells $)$ & 93 & 79 & 99 \\
\hline$\gamma \delta+(\mathrm{T}$ cells $)$ & 5 & 0.5 & 19 \\
\hline CD7- $(\mathrm{T}$ cells $)$ & 11 & 6 & 19 \\
\hline CD5- $(\gamma \delta$ T cells $)$ & 16 & 5 & 82 \\
\hline CD5-(CD8+ T cells) & 5.5 & 2 & 30 \\
\hline CD25+ $(\mathrm{T}$ cells $)$ & 32 & 18 & 52 \\
\hline NK cells (Lymphoid cells) & 7.7 & 4 & 18 \\
\hline CD2- (NK cells) & 36 & 23 & 53 \\
\hline Immature, CD56+br. CD16\&57- (NK cells) & 51 & 25 & 74 \\
\hline Mature, CD56+dim CD16\&57+br. (NK cells) & 29 & 13 & 63 \\
\hline Int., CD56+br. CD16\&57+dim (NK cells) & 10 & 4 & 23 \\
\hline B-cells (Lymphoid cells) & 46 & 30 & 67 \\
\hline CD10+ $($ B cells $)$ & 3.5 & 1 & 24 \\
\hline CD5+ (B cells $)$ & 8.5 & 1 & 15 \\
\hline$\kappa / \lambda$ ratio & 1.2 & 0.9 & 1.8 \\
\hline Plasma cells (Total cells) & 0.2 & 0.1 & 0.9 \\
\hline
\end{tabular}

